\title{
Effects of Strong and Electromagnetic Correlations on Neutrino Interactions in Dense Matter
}

\author{
Sanjay Reddy ${ }^{1,2}$, Madappa Prakash ${ }^{1}$, James M. Lattimer ${ }^{1}$, and Jose A. Pons ${ }^{3}$ \\ ${ }^{1}$ Department of Physics \& Astronomy, SUNY at Stony Brook, Stony Brook, New York 11794-3800 \\ 2 Institute For Nuclear Theory, University of Washington, Seattle, WA 98195 \\ 3 Department d'Astronomia, Universitat de València, E-46100 Burjassot, València, Spain
}

(October 26, 2018)

An extensive study of the effects of correlations on both charged and neutral current weak interaction rates in dense matter is performed. Both strong and electromagnetic correlations are considered. The propagation of particlehole interactions in the medium plays an important role in determining the neutrino mean free paths. The effects due to Pauli-Blocking and density, spin, and isospin correlations in the medium significantly reduce the neutrino cross sections. Due to the lack of experimental information at high density, these correlations are necessarily model dependent. For example, spin correlations in nonrelativistic models are found to lead to larger suppressions of neutrino cross sections compared to those of relativistic models. This is due to the tendency of the nonrelativistic models to develop spin instabilities. Notwithstanding the above caveats, and the differences between nonrelativistic and relativistic approaches such as the spin- and isospin-dependent interactions and the nucleon effective masses, suppressions of order $2-3$, relative to the case in which correlations are ignored, are obtained. Neutrino interactions in dense matter are especially important for supernova and early neutron star evolution calculations. The effects of correlations for protoneutron star evolution are calculated. Large effects on the internal thermodynamic properties of protoneutron stars, such as the temperature, are found. These translate into significant early enhancements in the emitted neutrino energies and fluxes, especially after a few seconds. At late times, beyond about 10 seconds, the emitted neutrino fluxes decrease more rapidly compared to simulations without the effects of correlations, due to the more rapid onset of neutrino transparency in the protoneutron star.

PACS numbers(s): 13.15.+g, 26.60.+c, 97.60.Jd 


\section{INTRODUCTION}

The calculation of neutrino interactions in hot, dense matter is highly relevant to the study of supernovae and protoneutron stars. Neutrinos are thought to be intimately involved in the supernova explosion mechanism. In addition, the timescales over which protoneutron stars deleptonize and cool, which are crucial in predicting the neutrino light curve from a supernova, are determined by neutrino opacities. In an earlier paper [1], we developed a formalism to determine both neutral and charged current opacities for interacting matter, accounting for in-medium mass and energy shifts given by its underlying equation of state (EOS). The effects of degeneracy and relativity were incorporated, and nuclear interactions modelled by both nonrelativistic potential and relativistic field-theoretical approaches were studied. In this paper, we extend this treatment to include important sources of suppression and enhancement due to in-medium correlations. In particular, we include effects due to both strong and electromagnetic correlations in the system. While neutrinos can couple to many-particle states and induce multi-pair excitations, both of which could modiy the response function developed in Ref. [1], single-pair excitations dominate over multi-pair excitations for the kinematics of interest in neutrino scattering and absorption. Thus, we focus on the effects of correlations on the single pair excitation spectrum.

Pioneering work in calculating neutrino mean free path in uniform nuclear matter was performed by Sawyer [2] who showed that the effects due to strong interactions are important and that the relation between the EOS and long wavelength excitations of the system may be exploited to compute the weak interaction rates consistent with the underlying dense matter model. Iwamoto and Pethick [3] computed the neutrino mean free paths in pure neutron matter for the densities of relevance for neutron stars by calculating the dynamical response within the framework of Fermi-liquid theory [4⿴囗十 [7]. Horowitz and Wehrberger [8] studied the influence of correlations on the neutrino scattering in dense matter in a relativistic fieldtheoretical model. More recently, Fabbri and Matera [9] have calculated neutrino scattering in asymmetric nuclear matter including the effects of the exchange interaction.

These earlier works on neutrino interactions in dense matter have shaped our approach. First accounts of our results may be found in Refs. [10,[1]. We urge the reader to consult the simultaneous and independent work of Burrows and Sawyer in Ref. [12], with which our work has some overlap. However, important differences, both in the microphysical inputs to the opacity calculations and in the underlying EOS models, distinguish our approach and results from theirs. We will present a unified approach to calculate both the neutral current scattering and charged current absorption reactions for the composition and temperatures of relevance to the PNS evolution. A systematic analysis of the various nuclear physics inputs such as the particle-hole (abbreviated p-h) interactions in density, spin and isospin channels is undertaken keeping in view the known nuclear ground state and excited state properties. We will isolate the important sources of enhancement and/or suppression due to in-medium correlations and highlight the important role of spin and isospin dependent p-h interactions. The need for further efforts to pin down the poorly known spin-dependent interactions is clearly brought out by our investigations.

The neutrino cross sections and the EOS are intimately related. This relationship is most transparent in the long-wavelength or static limit, in which the response of a system to a weak external probe is completely determined by the ground state thermodynamics 
(EOS). Thus, in this limit, neutrino opacities which are consistent with a given EOS [2] can be calculated. However, when the energy and momentum transferred by the neutrinos are large, full consistency is more difficult to achieve. Particle-hole and particle-particle interactions [10 12], multi-pair excitations [13], and the renormalization of the axial charge [14,15] are examples of effects that may influence both the EOS and the opacity. In this work, we investigate the influence of particle-hole excitations utilizing the Random Phase Approximation (RPA), in which ring diagrams are summed to all orders. This may be viewed as the first and minimal step towards achieving self-consistency between the response of matter to neutrino-induced perturbations and the underlying EOS, similar to what has long been established in the study of collective excitations in nuclei (multipole giant resonances) through electromagnetic probes [16].

Throughout this paper, we employ both nonrelativistic potential and relativistic fieldtheoretical models for the EOS and the evaluation of the neutrino-matter cross sections. This is done in order to evaluate effects that are generic as well as effects that are due to the precise description of the nucleon-nucleon interactions. In Sec. II, we study the response of pure neutron matter and calculate the neutrino cross sections to gauge the effects due to RPA correlations. In Sec. III, we calculate the influence of many-body correlations on the scattering and absorption reactions in both nuclear matter and multi-component stellar matter (charge neutral, $\beta$-equilibrated matter). In Sec. IV, we compute protoneutron star evolutions in order to evaluate the effects of correlations. Our conclusions and outlook are contained in Sec. V. Explicit formulae for the various p-h interactions and polarization functions needed to calculate the neutrino cross sections are collected in Appendices A and $\mathrm{B}$, respectively.

\section{NEUTRAL CURRENT NEUTRINO CROSS SECTIONS IN NEUTRON MATTER}

We begin with pure neutron matter to illustrate the various strong interaction correlation effects that influence the neutrino mean free paths in dense matter.

\section{A. Nonrelativistic Models}

In the nonrelativistic limit for the neutrons, the cross section per unit volume or the inverse mean free path for the neutral current reaction $\nu+n \rightarrow \nu+n$ is given by [3]

$$
\begin{aligned}
& \frac{1}{V} \frac{d^{3} \sigma\left(E_{1}\right)}{d \Omega^{2} d q_{0}}=\frac{G_{F}^{2}}{8 \pi^{3}} \quad E_{3}^{2} \quad\left[1-f_{\nu}\left(E_{3}\right)\right] \\
& \times\left[c_{V}^{2}(1+\cos \theta) S_{00}\left(q_{0}, q\right)+c_{A}^{2}(3-\cos \theta) S_{10}\left(q_{0}, q\right)\right],
\end{aligned}
$$

where $c_{V}=0.5$ and $c_{A}=0.615$ are the neutral current vector and axial vector couplings of the neutron and $G_{F}^{2}=6.89 \times 10^{-10} \mathrm{MeV}^{-5} \mathrm{~m}^{-1}, E_{1}$ and $E_{3}$ are the incoming and outgoing neutrino energies, $q_{0}=E_{1}-E_{3}$ and $\vec{q}=\overrightarrow{k_{1}}-\overrightarrow{k_{3}}$ are the energy and momentum transfers in the scattering reaction. The neutron matter response, which depends on the kinematical variables $q_{0}$ and $q=|\vec{q}|$, is characterized by the function $S\left(q_{0}, q\right)$ and is called the dynamic 
form factor. Since neutrinos couple to the vector and axial vector currents of the neutron, the response is given in terms of the density-density correlation function $S_{00}\left(q_{0}, q\right)$ and the spindensity correlation function $S_{10}\left(q_{0}, q\right)$. The subscripts appearing in the response function $S_{\sigma \tau}$ represent the total spin and isospin transferred along the p-h channel and are labelled as follows: $\tau=0$ and $\sigma=0$ for the neutral current density response, $\tau=0$ and $\sigma=1$ for the neutral current spin-density response, $\tau=1$ and $\sigma=0$ for the charged current density response, and $\tau=1$ and $\sigma=1$ for the charged current spin-density response. In the absence of strong interaction correlations, $S_{00}=S_{10}=S^{0}\left(q_{0}, q\right)$. The function $S^{0}\left(q_{0}, q\right)$ is directly related to the lowest order polarization function $\Pi^{0}$ (also known as the Lindhard function [17]) through the principle of detailed balance, and accounts for correlations arising due to Pauli blocking [18,19]. Explicitly,

$$
\begin{aligned}
S^{0}\left(q_{0}, q\right) & =\frac{\operatorname{Im} \Pi^{o}\left(q_{0}, q\right)}{1-\exp \left(-q_{0} / T\right)} \\
\Pi^{0}\left(q_{0}, q\right) & =\frac{2}{(2 \pi)^{3}} \int d^{3} p\left[\frac{f\left(E_{p}\right)\left[1-f\left(E_{|\vec{p}+\vec{q}|}\right)\right]}{q_{0}+E_{p}-E_{|\vec{p}+\vec{q}|}+i \epsilon}-\frac{\left[1-f\left(E_{p}\right)\right] f\left(E_{|\vec{p}+\vec{q}|}\right)}{q_{0}+E_{p}-E_{|\vec{p}+\vec{q}|}-i \epsilon}\right],
\end{aligned}
$$

where $f(E)=\left(1+\exp \left(E-\mu_{n}\right) / T\right)^{-1}$ is the Fermi-Dirac distribution function, $\mu_{n}$ is the neutron chemical potential, and $E_{p}=p^{2} / 2 M$ is the noninteracting nonrelativistic dispersion relation for the neutrons. The imaginary part of the polarization function may be explicitly evaluated when $q<2 k_{F}$ and $q_{0}<q$, and is given by [1,20]

$$
\begin{aligned}
\operatorname{Im} \Pi^{0}\left(q_{0}, q\right) & =\frac{M^{2} T}{2 \pi q} \quad\left[\frac{q_{0}}{T}+\ln \left(\frac{1+\exp \left[\left(e_{-}-\mu_{2}\right) / T\right]}{1+\exp \left[\left(e_{-}+q_{0}-\mu_{4}\right) / T\right]}\right)\right], \\
e_{-} & =\frac{p_{-}^{2}}{2 M}=\frac{1}{4} \frac{\left(q_{0}-q^{2} / 2 M\right)^{2}}{q^{2} / 2 M} .
\end{aligned}
$$

The total cross section per unit volume or the inverse collision mean free path of neutrinos due to scattering is obtained by integrating over the $q_{0}-q$ space.

The simplest modification to $S\left(q_{0}, q\right)$ due to strong interactions arises due to the medium modification of the single particle dispersion relation [1]. In the mean field approximation (in which particles are assumed to move independently of each other in a common potential), the single particle spectrum may be cast into the form

$$
E(p)=p^{2} / 2 M^{*}+U
$$

in schematic models which are designed to reproduce the results of microscopic calculations. The single particle potential $U$ and the (Landau) effective mass $M^{*}$ are in general density dependent. Because the functional dependence of the spectra on the momenta is similar to that of the noninteracting case, the only modification to the free gas results arises due to the nucleon effective mass. Thus, to obtain the mean field or Hartree response we need only replace the free nucleon mass $M$ in Eq. (3) by the in-medium mass $M^{*}$. The density dependence of $M^{*}$ is model dependent and in nonrelativistic approaches arises due to the presence of a momentum dependent interaction. Modifications due to $M^{*}\left(n_{B}\right)$ are important and always act to decrease the scattering cross sections. This is chiefly due to the fact that 
a lower $M^{*}$ decreases the density of states $N_{0}=M^{*} k_{F} / \pi^{2}$ near the Fermi surface and thereby the number of target neutrons available for scattering. Kinematically, a lower $M^{*}$ favors larger energy transfers by shifting the strength from low $q_{0}$ to regions of high $q_{0}$. For thermal neutrinos, the dominant contribution to the total cross section comes from the region $\left|q_{0}\right| \leq \pi T$, since factors arising from the principle of detailed balance and final state blocking for the neutrinos exponentially suppress the phase space when $\left|q_{0}\right| \geq \pi T$. Thus, for partially degenerate matter and for neutrino energies of order $T$, this shift in the strength leads to significant suppressions in the total cross sections.

The results in Fig. 1 show how $M^{*}\left(n_{B}\right)$, which depends on the strong interaction model, modifies the neutrino mean free path as a function of density for different temperatures. For the illustrative results presented here, we have used $M^{*} / M=\left[1+\alpha\left(n_{B} / n_{0}\right)\right]^{-1}$, characteristic of a large class of Skyrme models for $n_{B} \lesssim(3-4) n_{0}$, where $n_{0}=0.16 \mathrm{fm}^{-3}$ is the nuclear saturation density. Denoting the value of the nucleon mass at $n_{0}$ by $M_{0}^{*}$, the factor $\alpha=$ $\left(M-M_{0}^{*}\right) / M_{0}^{*}$. For the results shown in Fig. 1, we have set $M_{0}^{*}=0.8$. Our findings indicate that the neutrino mean free paths are roughly enhanced by a factor proportional to $\left(M / M^{*}\right)^{2}$. With increasing temperature, this enhancement decreases only moderately. We are thus led to the conclusion that for the densities and temperatures of interest, effects due to a dropping in-medium nucleon mass significantly increases the neutrino mean free path.

We turn now to address the effects of strong interaction correlations. We first note that the nucleon-nucleon interaction for densities of relevance is not amenable to a perturbative treatment [21,22]. Thus, we are forced to adopt approximate schemes to incorporate nonperturbative effects. The random phase approximation (RPA), also known as the ring approximation, is particularly suited to describe the response of matter at high densities. In RPA, ring diagrams are summed to all orders in order to incorporate p-h correlations. Single pair excitations created by the neutrinos are now allowed to propagate in the medium due to the presence of $\mathrm{p}-\mathrm{h}$ interactions. The strength of the interaction in a specific $\mathrm{p}-\mathrm{h}$ channel will therefore play a crucial role in determining the magnitude of the RPA corrections. Deferring to later sections the discussion of the interaction potentials, we set up here the basic formalism to calculate the cross sections for a given interaction $V_{00}$ in the spin independent channel and $V_{10}$ in the spin dependent channel. The RPA polarization, as a result of summing ring diagrams to all orders, is [18

$$
\Pi_{i j}^{R P A}=\frac{\Pi^{0}}{1-V_{i j} \Pi^{0}} .
$$

The dynamic form factor which incorporates these correlations is given by

$$
\begin{aligned}
& S_{i j}\left(q_{0}, q\right)=\left[\frac{1}{1-\exp \left(q_{0} / T\right)}\right] \frac{\operatorname{Im} \Pi^{o}\left(q_{0}, q\right)}{\epsilon_{i, j}}, \\
& \epsilon_{i, j}\left(q_{0}, q\right)=\left[1-V_{i j} \operatorname{Re} \Pi^{0}\left(q_{0}, q\right)\right]^{2}+\left[V_{i j} \operatorname{Im} \Pi^{o}\left(q_{0}, q\right)\right]^{2} .
\end{aligned}
$$

In addition to the imaginary part of the polarization, which is sufficient to evaluate the free gas response, the real part is also needed to evaluate the corrections due to strong interaction correlations. At finite temperatures, the real part is easily evaluated numerically by using the Kramers-Kronig relation

$$
\operatorname{Re} \Pi^{o}\left(q_{0}, q\right)=-\frac{1}{\pi} \mathcal{P} \int_{-\infty}^{\infty} d \omega \quad \frac{\operatorname{Im} \Pi^{o}(\omega, q)}{\omega-q_{0}} .
$$


The dielectric screening function $\epsilon_{i, j}$ is the only modification that arises in RPA. If the interaction is repulsive $\epsilon_{i, j}\left(q_{0}=0, q\right)>1$, the medium response is suppressed, while an attractive interaction will result in an enhancement. If the repulsive interaction is strong, the RPA response predicts the existence of collective excitations such as zero-sound and spin zero-sound which could enhance the response when the energy transfer is finite and equal to the energy of the collective state.

\section{Particle-Hole Interactions}

The particle-hole (abbreviated as p-h) interaction, $V_{i j}$, is in general a function of density, temperature, $q_{0}$, and $q$. For the temperatures of interest in the PNS evolution, $q_{0}$ and $q$ are typically smaller than a few tens of MeV's. Since the p-h interactions due to strong interactions are short ranged $(\sim 1$ /meson mass) compared with the typical wavelength of the excitations probed by the neutrinos, the momentum dependence of the interaction will not play a significant role. It is well known that the effective interaction of meson exchange models result in very large matrix elements due to the presence of a large short-ranged component and cannot be used in perturbative expansion [21,22]. In nonrelativistic models, there are essentially two complementary methods that have been used to estimate the p-h interactions in nuclei and in bulk nuclear matter. We outline the basic features of these methods and employ the corresponding $\mathrm{p}$-h interaction potentials to compute the neutrino scattering cross sections.

\section{Schematic Potential Models}

Skyrme-type models start from a zero-range, density, and momentum dependent potential from which an energy density functional is constructed. The force parameters are determined empirically by calculating the ground state in the Hartree-Fock approximation and by fitting the observed ground state properties of nuclei and nuclear matter. Using Landau Fermi-liquid theory, the effective p-h interaction is given by the second functional derivative of the total energy density with respect to the densities taken at the Hartree-Fock solution: $V_{i j}=\left(\delta^{2} E(n) / \delta n_{i} \delta n_{j}\right)$, and is usually expressed in terms of Fermi-liquid parameters. When the interaction is short ranged compared to the wavelength of the excitations of interest, justifying the assumption that only $l=0$ terms are retained, $V_{i j}$ assumes a simple form. For example, in symmetric nuclear matter the p-h interaction is given by [21]

$$
\mathrm{V}\left(k_{1}, k_{2}\right)=N_{0}^{-1}\left[F_{0}+G_{0} \sigma_{\mathbf{1}} \cdot \sigma_{\mathbf{2}}+\left(F_{0}^{\prime}+G_{0}^{\prime} \sigma_{\mathbf{1}} \cdot \sigma_{\mathbf{2}}\right) \tau_{\mathbf{1}} \cdot \tau_{\mathbf{2}}\right],
$$

where $\sigma$ and $\tau$ are spin and isospin Pauli matrices, which act on the p-h states. To achieve full generality, Eq. (8) should be supplemented with contributions from tensor interactions. The Fermi-liquid parameters $F_{0}, G_{0}, F_{0}^{\prime}$, and $G_{0}^{\prime}$ are dimensionless numbers and $N_{0}=2 M^{*} k_{F} / \pi^{2}$ is the density of states at the Fermi surface. In neutron matter, since we have frozen the isospin degree of freedom, only the first two terms contribute (with $N_{0}^{-1}=M^{*} k_{F} / \pi^{2}$ ), and the effective interaction $V_{00}=N_{0}^{-1} F_{0}$ and $V_{10}=N_{0}^{-1} G_{0}$. The nucleon effective mass $M^{*}$, which arises due to a momentum dependent interaction, is given by $M^{*} / M=\left(1+F_{1} / 3\right)$. 
Skyrme models have been successful in describing nuclei and their excited states [16. In addition, various authors have explored its applicability to describe bulk matter at densities of relevance to neutron stars. The effective nucleon-nucleon interaction of the standard Skyrme model is given by the potential 23]

$$
\begin{aligned}
V_{N N}(r) & =t_{0}\left(1+x_{0} P_{\sigma}\right) \delta(r)+\frac{1}{6} t_{3} n^{\gamma}\left(1+x_{3} P_{\sigma}\right) \delta(r) \\
& +\frac{1}{2} t_{1}\left(1+x_{1} P_{\sigma}\right)\left[\hat{k}^{2} \delta(r)+\hat{k}^{\dagger} \delta(r)\right]+t_{2}\left(1+x_{2} P_{\sigma}\right) \hat{k^{\dagger}} \delta(r) \hat{k},
\end{aligned}
$$

where, the force parameters $t_{0}, t_{1}, t_{2}, t_{3}$, and the exchange force parameters $x_{0}, x_{1}, x_{2}$, and $x_{3}$ are empirically determined. The three-body interaction, which is written as a density dependent two-body interaction (through the parameters $t_{3}$ and $\gamma$ ) and the momentum dependent interactions arising due to $t_{1}$ and $t_{2}$ terms dominate the high density behavior of these models.

The Fermi-liquid parameters for neutron matter, expressed explicitly in terms of the Skyrme parameters, are collected in Appendix A. Fig. 2 shows the results for four different sets of Skyrme parameters for $n_{B} \lesssim 4 n_{0}$. A striking feature is the large density dependence of the Fermi-liquid parameters. While the qualitative behaviors of all four parameter sets are similar, significant quantitative differences exist. The models SkM* and SGII 24 have been constrained by fitting the properties of systems with very small isospin asymmetries, while the models SLy4 and SLy5 were further constrained to reproduce the results of microscopic neutron matter calculations 25]. All four models become unstable to spin oscillations and are driven towards a ferromagnetic ground state since $G_{0}>-1$ for densities in the range $(2-4) n_{0}$. For $0>G_{0}>-1$, the RPA corrections enhance the spin response approximately by a factor $\left(1+G_{0}\right)^{-2}$ resulting in small neutrino mean free paths. For $G_{0}<-1$, the spin symmetric ground state is unstable. At the phase transition density, RPA response functions of the spin symmetric state diverge, and the formalism developed thus far is inapplicable. The response functions of the energetically favored, spin polarized ground state needs to be calculated. We do not attempt to do this here; we merely note that large enhancements in the RPA response functions are precursors to a phase transition. We present results only for $n_{B}<2 n_{0}$, for which the ground state is spin symmetric. Spin instability is a common feature associated with a large class of Skyrme models [26], but is not realized in more microscopic calculations. It must be emphasized that the interaction in the spin dependent channel is a crucial ingredient in calculating the response functions. A "poorly" behaved spin interaction introduces a significantly large model dependence in the neutrino cross sections.

$F_{0}$, on the other hand, is well behaved and is qualitatively similar to the results of microscopic calculations. This is expected, since interactions in this channel are fit to the empirical value of the nuclear compressibility $K$ at $n_{0}$. The models SLy4 and SLy $5\left(K_{0}=230\right.$ $\mathrm{MeV}$ ) were further constrained to reproduce the pressure versus density curve for pure neutron matter of the microscopic calculations of Wiringa et al. [27]. To date, spin dependent interactions in the Skyrme models have not been directly constrained. This accounts for the large differences between the various models. Whether or not a simple Skyrme model can mimic the bulk properties of a microscopic calculation of both spin symmetric and spin asymmetric systems remains an open question. For this reason, the use of a Skyrme model to describe the spin response of neutron matter for densities of interest in neutron stars does not appear to be promising until the spin dependent interaction is constrained. Nevertheless, 
the energy density functional method provides a consistent framework to study the response of hot and dense asymmetric nuclear matter. We hope that further studies will provide a better Skyrme energy density functional or an alternative parameterization that will be useful for neutron star calculations.

\section{Microscopic Potential Models}

In a microscopic approach, one starts with the bare interaction and obtains an effective interaction by solving iteratively the Bethe-Goldstone equation. Commonly known as the Brueckner G-matrix method, this method provides a useful means of arriving at density dependent effective p-h interactions. Further refinements include the use of correlated basis states and inclusion of a larger class of diagrams [28]. In this article, we do not wish to address the merits and demerits of these calculations; instead, we contrast the neutron matter results obtained by Backmann et al. [29] with those of Jackson et al. 28] for the Reid- $v_{6}$ and Bethe-Johnson- $v_{6}$ potentials (see Fig. 3). In sharp contrast to the Skyrme models, these calculations predict a large and repulsive spin interaction $\left(G_{0}>1\right)$. Although the qualitative behavior of $F_{0}$ and $M^{*}$ are similar, important quantitative differences exist. In view of this, a knowledge of at least the $\ell=0$ Fermi-liquid parameters of more modern calculations 30,31] would be very useful.

\section{Neutral Current Neutrino Scattering Cross Sections}

In the density regions where spin stability is assured, the neutrino mean free paths may be computed for the Skyrme models in degenerate neutron matter, since $M^{*}, V_{00}$, and $V_{10}$ are easily expressible in terms of the Skyrme parameters (see Appendix A). The models SLy4 and SLy5 have been constrained to better fit systems with large isospin asymmetries. For this reason, we choose the model SLy4 to study the neutron matter response. Fig. 4 shows the neutrino differential cross section for a neutrino energy $E_{\nu}=25 \mathrm{MeV}$, at fixed momentum transfer $q=25 \mathrm{MeV}$, as a function of $q_{0} / q$. At $n_{B}=0.04 \mathrm{fm}^{-3}$, the p-h interaction is attractive in the density-density channel $\left(F_{0}=-0.7\right)$ and repulsive in the spin-density channel $\left(G_{0}=0.6\right)$. This accounts for the enhancement (over the Hartree result) in the vector channel and suppression in the axial vector channel at small $q_{0}$. The enhancement in the spin channel when $q_{0} / q \sim v_{F}$, where $v_{F}=k_{F} / M^{*}$ is the velocity at the

Fermi surface, is due to the presence of a collective spin excitation that arises due to the large and repulsive spin interaction. At finite temperatures, the response is dominated by the kinematical region in which $q_{0}<T$.

The bottom right panels of Fig. 14 show that a strong and attractive interaction in the vector channel gives rise to a large peak in the vector response which dominates the total differential cross section. The weak collective spin state seen at $\mathrm{T}=0$ is almost completely damped at finite temperatures and only modestly enhances the cross sections in this kinematical region.

The top panels of Fig. 1 show results for $n_{B}=0.16 \mathrm{fm}^{-3}$. For densities close to $n_{0}$, the p-h interaction in both channels is relatively weak. It remains repulsive in the spin channel $\left(G_{0} \cong 0.15\right)$ and attractive in the spin independent channel $\left(F_{0} \cong-0.28\right)$. The 
RPA correlations do not significantly alter the differential cross sections since the modest suppression in the spin response is compensated by an enhancement in the density response. At higher densities, the Skyrme models investigated here predict a phase transition to a ferromagnetic state and become acausal. In our view, these are clear indicators of an inherent high density problem in the Skyrme models due to the very strong momentum and density dependence of the bare interaction. The large differences in the density dependence of the Fermi-liquid parameters for the different models suggest that this is indeed the case.

In Fig. 5, the neutrino differential cross sections for the neutron matter EOS of Backmann et al. [29] are shown. At all relevant densities, the repulsive spin interaction accounts for the suppression in the axial response and also for the enhancement in the region where $q_{0} / q \sim v_{F}$ due to the presence of a collective spin resonance. The vector part is enhanced at low density and suppressed at higher densities as $F_{0}$ becomes positive. At $2 n_{0}$, the repulsion in this channel is sufficient to produce a weakly damped zero-sound mode. The total differential cross sections are significantly suppressed in all cases, and the suppression increases with increasing density.

The collision mean free paths for the Skyrme model SLy4 and for the microscopic model of Bäckmann et al. [29] are compared in Fig. 6. The mean free paths with and without RPA corrections are labelled $\lambda_{R P A}$ and $\lambda_{M *}$, respectively. The qualitative trends are similar to those observed at the level of the differential cross sections. The ratio $\lambda_{R P A} / \lambda_{M *}$ shown in the right panels indicate that typical RPA corrections are large, but model dependent. Neutrino mean free paths are suppressed in the model SLy4 due to an attractive p-h interaction. When the Fermi-liquid parameters obtained by Bäckmann et al. 229] are employed, the large and repulsive spin interactions enhance the mean free path by as much as a factor of four. The differences between the Hartree results $\left(\lambda_{M^{*}}\right)$ reflect the different density dependencies of the nucleon effective mass predicted by these models (see Fig. 2 and Fig. [). The density dependence of $M^{*}$ in the Skyrme model SLy4 is very strong, but is relatively moderate in the microscopic calculation due to Bäckmann et al. [29]. The increase in the Hartree mean free paths with increasing density is a result of the rapidly decreasing nucleon effective mass and the use of a nonrelativistic dispersion relation. At high density, a nonrelativistic description for the nucleon kinematics breaks down and results in a spurious increase in the neutrino mean free path. In the next section, we study the response of matter in relativistic models in which some of these deficiencies are remedied.

\section{B. Relativistic Models}

In Walecka-type relativistic field-theoretical models, nucleons are described as Dirac spinors which interact via the exchange of mesons. In the simplest of these models, the $\sigma$ and $\omega$-meson fields simulate the attractive and repulsive character of the nucleon-nucleon interaction, respectively. The strengths of the nucleon-meson couplings are fixed by the ground state properties of nuclear matter at saturation density by solving the field theory at a specified level of approximation [32]. Further, to account for the isovector character of the nucleon-nucleon interaction, the $\rho$-meson contribution is included and the $\rho N N$ coupling is fixed by the empirical value of the nuclear symmetry energy. In this section, we study the response of neutron matter whose ground state properties are calculated in the mean field approximation to the $\sigma \omega \rho$ model. 
The differential cross section per unit volume $V$ for $\nu$-neutron scattering is given by [33]

$$
\frac{1}{V} \frac{d^{3} \sigma}{d^{2} \Omega_{3} d E_{3}}=-\frac{G_{F}^{2}}{32 \pi^{2}} \frac{E_{3}}{E_{1}}\left[1-\exp \left(-\frac{q_{0}}{T}\right)\right]^{-1}\left[1-f_{3}\left(E_{3}\right)\right] \times \operatorname{Im}\left(L^{\alpha \beta} \Pi_{\alpha \beta}^{R}\right),
$$

where $E_{1}$ and $E_{3}$ are the incoming and outgoing neutrino energies and $q_{0}=E_{1}-E_{3}$ is the energy transfer in the reaction. In terms of the incoming neutrino four momentum $k=\left(E_{1}, \vec{k}\right)$ and the four momentum transfer $q_{\mu}=\left(q_{0}, \vec{q}\right)$, the lepton tensor $L_{\alpha \beta}$ is given by

$$
L^{\alpha \beta}=8\left[2 k^{\alpha} k^{\beta}+(k \cdot q) g^{\alpha \beta}-\left(k^{\alpha} q^{\beta}+q^{\alpha} k^{\beta}\right) \mp i \epsilon^{\alpha \beta \mu \nu} k^{\mu} q^{\nu}\right] .
$$

The target particle retarded polarization tensor, which is a function of the neutron chemical potential, temperature, the kinematical variables $q_{0}$, and $|\vec{q}|$, is

$$
\operatorname{Im} \Pi_{\alpha \beta}^{R}=\tanh \left(\frac{q_{0}}{2 T}\right) \operatorname{Im} \Pi_{\alpha \beta}
$$

where $\Pi_{\alpha \beta}$ is the time ordered or causal polarization and is given by

$$
\Pi_{\alpha \beta}=-i \int \frac{d^{4} p}{(2 \pi)^{4}} \operatorname{Tr}\left[T\left(G_{2}(p) J_{\alpha} G_{4}(p+q) J_{\beta}\right)\right]
$$

The Green's functions $G_{i}(p)$ (the index $i$ labels particle species) describe the propagation of baryons at finite density and temperature. The current operator $J_{\mu}$ is $\gamma_{\mu}$ for the vector current and $\gamma_{\mu} \gamma_{5}$ for the axial current. Given the structure of the particle currents, we have

$$
\Pi_{\alpha \beta}=c_{V}^{2} \Pi_{\alpha \beta}^{V}+c_{A}^{2} \Pi_{\alpha \beta}^{A}-2 c_{V} c_{A} \Pi_{\alpha \beta}^{V A} .
$$

For the vector polarization, $\left\{J_{\alpha}, J_{\beta}\right\}::\left\{\gamma_{\alpha}, \gamma_{\beta}\right\}$, for the axial polarization, $\left\{J_{\alpha}, J_{\beta}\right\}$ :: $\left\{\gamma_{\alpha} \gamma_{5}, \gamma_{\beta} \gamma_{5}\right\}$, and for the mixed part, $\left\{J_{\alpha}, J_{\beta}\right\}::\left\{\gamma_{\alpha} \gamma_{5}, \gamma_{\beta}\right\}$. Using vector current conservation and translational invariance, $\Pi_{\alpha \beta}^{V}$ may be written in terms of two independent components. In a frame where $q_{\mu}=\left(q_{0},|q|, 0,0\right)$, we have

$$
\Pi_{T}=\Pi_{22}^{V} \quad \text { and } \quad \Pi_{L}=-\frac{q_{\mu}^{2}}{|q|^{2}} \Pi_{00}^{V} .
$$

The axial current correlation function can be written as a vector piece plus a correction term:

$$
\Pi_{\mu \nu}^{A}=\Pi_{\mu \nu}^{V}+g_{\mu \nu} \Pi^{A}
$$

The mixed axial vector current correlation function is

$$
\Pi_{\mu \nu}^{V A}=i \epsilon_{\mu, \nu, \alpha, 0} q^{\alpha} \Pi^{V A}
$$

The above mean field or Hartree polarizations, which characterize the medium response to the neutrino, have been explicitly evaluated in previous works [34,35] and are collected in Appendix B. At the Hartree level, the only modification that arises is due to the density dependent nucleon effective mass. 
In the $\sigma \omega \rho$ model, $\mathrm{p}-\mathrm{h}$ excitations propagate in the medium via the interactions arising due to these mesons. The RPA response includes a subclass of these excitations; namely, the ring diagrams are summed to all orders by solving the Dyson equation [8]. To calculate the required polarizations, we begin with the Lagrangian density

$$
\begin{aligned}
L & =\sum_{B} \bar{B}\left(-i \gamma^{\mu} \partial_{\mu}-g_{\omega B} \gamma^{\mu} \omega_{\mu}-g_{\rho B} \gamma^{\mu} \mathbf{b}_{\mu} \cdot \mathbf{t}-M_{B}+g_{\sigma B} \sigma\right) B \\
& -\frac{1}{4} W_{\mu \nu} W^{\mu \nu}+\frac{1}{2} m_{\omega}^{2} \omega_{\mu} \omega^{\mu}-\frac{1}{4} \mathbf{B}_{\mu \nu} \mathbf{B}^{\mu \nu}+\frac{1}{2} m_{\rho}^{2} b_{\mu} b^{\mu}+\frac{1}{2} \partial_{\mu} \sigma \partial^{\mu} \sigma-\frac{1}{2} m_{\sigma}^{2} \sigma^{2}-U(\sigma) \\
& +\sum_{l} \bar{l}\left(-i \gamma^{\mu} \partial_{\mu}-m_{l}\right) l .
\end{aligned}
$$

Here, $B$ are the Dirac spinors for baryons and $\mathbf{t}$ is the isospin operator. The field strength tensors for the $\omega$ - and $\rho$-mesons are $W_{\mu \nu}=\partial_{\mu} \omega_{\nu}-\partial_{\nu} \omega_{\mu}$ and $\mathbf{B}_{\mu \nu}=\partial_{\mu} \mathbf{b}_{\nu}-\partial_{\nu} \mathbf{b}_{\mu}$, respectively, and $U(\sigma)$ represents the scalar self-interactions and is taken to be of the form $U(\sigma)=$ $(b / 3) M_{n}\left(g_{\sigma N} \sigma\right)^{3}+(c / 4)\left(g_{\sigma N} \sigma\right)^{4}$. The model may then be solved either in the mean field approximation (MFT) or in the relativistic Hartree approximation (RHA). In either case, the nucleon propagator $G\left(p_{\mu}\right)$ retains the same structure as in the noninteracting case, but with constant density dependent shifts to the mass and energy due the presence of scalar and vector mean fields. Explicitly, $M \rightarrow M^{*}=M-g_{\sigma} \sigma$ and $p_{0} \rightarrow p_{0}^{*}=p_{0}-g_{\omega} \omega+\frac{1}{2} g_{\rho} b$, where $\sigma, \omega$, and $b$ are the zeroth components of the $\sigma-, \omega-$, and $\rho$-meson mean fields. The Hartree polarization functions (see Appendix B) are only sensitive to mass shifts since the momentum independent energy shifts exactly cancel. The Dyson equations for the various polarizations are

$$
\tilde{\Pi}^{\mu, \nu}=\Pi^{\mu, \nu}+\tilde{\Pi}^{\mu, \tau} D_{\tau, \gamma} \Pi^{\gamma, \nu},
$$

where $\tilde{\Pi}^{\mu, \nu}$ is the RPA polarization function and $D_{\tau, \gamma}$ is the interaction due to the exchange of scalar and vector mesons. The RPA response in these field-theoretical models have been studied previously [33]. Here, we gather the important steps leading to the evaluation of the RPA corrections. The correction to the vector part of the response is given by

$$
\delta \Pi_{V}^{\mu, \nu}=\tilde{\Pi}^{\mu, \nu}-\Pi^{\mu, \nu} .
$$

In order to account for the mixing between the attractive scalar and the repulsive vector interactions, the Dyson equation in the isoscalar longitudinal channel becomes

$$
\left[\begin{array}{cc}
\tilde{\Pi}^{S} & \tilde{\Pi}^{M} \\
\tilde{\Pi}^{M} & \tilde{\Pi}^{00}
\end{array}\right]=\left[\begin{array}{cc}
\Pi^{S} & \Pi^{M} \\
\Pi^{M} & \Pi^{00}
\end{array}\right]+\left[\begin{array}{cc}
\tilde{\Pi}^{S} & \tilde{\Pi}^{M} \\
\tilde{\Pi}^{M} & \tilde{\Pi}^{00}
\end{array}\right]\left[\begin{array}{cc}
-\chi_{S} & 0 \\
0 & -\tilde{\chi}_{V}
\end{array}\right]\left[\begin{array}{cc}
\Pi^{S} & \Pi^{M} \\
\Pi^{M} & \Pi^{00}
\end{array}\right]
$$

The interaction matrix is written in terms of the variables

$$
\chi_{S}=\frac{\left(g_{\sigma} / m_{\sigma}\right)^{2}}{1-q_{\mu}^{2} / m_{\sigma}^{2}}, \quad \chi_{\omega}=\frac{\left(g_{\omega} / m_{\omega}\right)^{2}}{1-q_{\mu}^{2} / m_{\omega}^{2}}, \quad \chi_{\rho}=\frac{\left(g_{\rho} / m_{\rho}\right)^{2}}{1-q_{\mu}^{2} / m_{\rho}^{2}},
$$

and $\tilde{\chi}_{V}=q_{\mu}^{2} \chi_{V} / q^{2}$, where $\chi_{V}=\chi_{\omega}+\chi_{\rho} / 4$ is the sum of $\omega$ - and $\rho$-meson contributions to the vector interaction. Note that the $\rho$-meson contribution to the interaction is significantly smaller than that of the $\omega$-meson and provides a very small additional repulsion. The 
dominant contributions arise due to $\sigma$ - and $\omega$-mesons. The solution to the above matrix equation yields

$$
\begin{aligned}
\delta \Pi_{V}^{00} & =-\left[\frac{\tilde{\chi}_{V} \Pi_{00}^{2}+\chi_{S} \Pi_{M}^{2}-\tilde{\chi}_{V} \chi_{S}\left(\Pi_{M}^{2}-\Pi_{00} \Pi_{S}\right)}{\epsilon_{L}}\right] \\
\epsilon_{L} & =1+\chi_{S} \Pi_{S}+\tilde{\chi}_{V} \Pi_{00}+\tilde{\chi}_{V} \chi_{S}\left(\Pi_{M}^{2}-\Pi_{00} \Pi_{S}\right) .
\end{aligned}
$$

Only the vector mesons contribute to the transverse parts and the RPA corrections in this case take the simple form

$$
\delta \Pi_{T}=\delta \Pi_{22}=\frac{\Pi_{T} \chi_{V} \Pi_{T}}{\epsilon_{T}} ; \quad \epsilon_{T}=1+\chi_{V} \Pi_{T} .
$$

The axial vector polarization is not significantly modified in the $\sigma \omega \rho$ model, since the vector mesons modify the axial response only through the vector-axial vector mixing. The corrections to the axial vector response due to the correlations induced by the vector mesons are given by the relations

$$
\tilde{\Pi}_{A}^{\mu \nu}=\Pi_{A}^{\mu \nu}+\Pi_{V} \chi_{V} \tilde{\Pi}_{V A} \quad \text { and } \quad \tilde{\Pi}_{V A}=\Pi_{V A}+\tilde{\Pi}_{V A} \chi_{V} \Pi_{T}
$$

To directly modify the axial response, we require a meson with a pseudo-vector coupling to the nucleons. The lightest meson which may be coupled to the nucleons in this way is the pion. However, the direct part of the pion contribution in this channel will be suppressed in the long wavelength limit, since the derivative $\pi N N$ coupling vanishes in the limit $q_{\mu} \rightarrow 0$. In addition, it is well known from nuclear phenomenology that there exists a large repulsive component in the spin-isospin channel. To account for this large repulsion, various authors (see, for example, Ref. [21]) have suggested the use of a very short range repulsive interaction parametrized through the so-called Migdal parameter $g^{\prime}$. This is usually accomplished by modifying the pion propagator according to [36]

$$
\frac{q_{\mu} q_{\nu}}{q_{\mu}^{2}-m_{\pi}^{2}} \rightarrow \frac{q_{\mu} q_{\nu}}{q_{\mu}^{2}-m_{\pi}^{2}}-g^{\prime} g_{\mu \nu}
$$

The value of $g^{\prime}$ is expected to be in the range (0.5-0.7) in nonrelativistic models. However, a detailed study to empirically determine this value in relativistic models does not exist. For this reason, we introduce a point-like interaction with a pseudo-vector coupling to the nucleons and parameterize its strength through the Migdal parameter $g^{\prime}$. The strength of the coupling is given by $\chi_{A}=g^{\prime} f_{\pi}^{2} / m_{\pi}^{2}$. The pion contribution, which is expected to be attractive albeit small, is not explicitly taken into account, since we are treating $g^{\prime}$ as a parameter that characterizes the total strength in the pseudo-vector channel. With this choice, the RPA corrections to the axial vector polarization function is given by

$$
\delta \Pi_{A}^{\mu \nu}=\Pi_{A}^{\mu \tau} \tilde{D}_{A}^{\tau \gamma} \Pi_{A}^{\gamma \nu} \quad \text { with } \quad \tilde{D}_{A}=\left(1-D_{A}\right)^{-1} D_{A} \quad \text { and } \quad D_{A}=\chi_{A} g_{\mu \nu} .
$$

Having determined the correlated polarization functions, we may calculate the differential cross section in a relativistic model. For this purpose, we have chosen a mean field model labelled GM3 and a model which includes the vacuum contributions incorporated through the relativistic Hartree approximation (RHA). The various coupling constants for the model GM3 and a detailed discussion of these field theoretical models can be found in Ref. [1]. 


\section{Neutrino Scattering Cross Sections}

We begin by discussing the results of neutrino mean free paths obtained when RPA correlations are ignored (Fig. (7). In this case, the only medium dependence arises through the density dependent $M^{*}$. With increasing density, the decreasing $M^{*}$ nearly compensates for the increase in particle density so as to make the neutrino mean free path approach a constant value at high density. This feature, seen in both field-theoretical models, is not seen in the free gas case, in which the neutrino mean free paths decrease far more rapidly. The nonrelativistic cross sections, which are roughly proportional to $M^{*^{2}}$, are reduced significantly with decreasing $M^{*}$. In the relativistic case, the cross sections are proportional to $E_{F}^{2}=k_{F}^{2}+M^{*^{2}}$, resulting in a relatively weaker density dependence. Thus, at high densities of interest in neutron stars, the use of nonrelativistic reaction kinematics underestimates the cross sections by approximately a factor $\left(M^{*^{2}} / E_{F}^{2}\right)$ due to the decreasing behavior of $M^{*}$ with density. This factor could be as small as 0.5 at high density.

RPA corrections in models GM3 and RHA due to the $\sigma, \omega, \rho$, and $g^{\prime}$ correlations suppress the cross sections at high density owing to the repulsive character of these interactions. At lower density, when $M^{*} \gg k_{F}$, the mixing between the scalar and vector mesons is large and the interaction in the longitudinal vector channel is attractive due to the dominant contribution of the scalar meson. At higher densities, mixing becomes unimportant and vector meson contributions dominate leading to significant suppression. The transverse components of the response in the vector channel are reduced at all densities, since only the vector mesons contribute to this correlation function. In Fig. 8 and Fig. 9, the differential cross sections with and without correlations are shown for the RHA and GM3 models. In the absence of pseudo-vector correlations arising due to $g^{\prime}$, only the vector part is modified.

The correction to the axial vector response functions are negligibly small when $g^{\prime}=0$. The corrections to the vector part are significant at high density (more than a factor of two). However, since the axial current contributions dominate the differential cross sections, even large suppressions to the vector part of the response will translate to only modest changes in the neutrino scattering rates. To explore the sensitivity to the magnitude of $g^{\prime}$, we study the response for $g^{\prime}=0.3$ and $g^{\prime}=0.6$. Effects due to repulsive correlations are quite significant even for $g^{\prime}=0.3$ (see Fig. 8 and Fig. 9).

Fig. 10 shows the mean free paths for the models investigated in this work. The left panels show the Hartree cross sections and the right panels show the extent to which RPA correlations modify the Hartree results as a function of both density and temperature. RPA correlations due to $\sigma-, \omega-$, and $\rho$-mesons result in $(20-30) \%$ increase in the neutrino mean free paths over the Hartree results at high density. These modifications are modest and show little density dependence, since they predominantly act only on the vector part of the neutron matter response. The pseudo-vector corrections modify the dominant axial vector response and significantly increase the mean free path. With increasing density, these corrections first increase and then decrease due to the small nucleon effective mass at high density. 


\section{NEUTRAL CURRENT NEUTRINO CROSS SECTIONS IN MULTI-COMPONENT MATTER}

The response of multi-component matter differs in several respects from that of a single component system. At the free gas level, the neutrino cross sections are influenced by compositional effects arising due to species specific neutrino-matter couplings, and more importantly due to a reduction in the Pauli-blocking. At fixed baryon density and temperature, additional baryonic components will significantly decrease the degree of particle degeneracy and enhance the scattering cross sections. In charge neutral matter containing nucleons and leptons in $\beta$-equilibrium, the proton fraction depends very sensitively on the dense matter EOS through the density dependent nuclear symmetry energy. The role of the nuclear symmetry energy in determining the neutrino scattering and absorption mean free paths calculated in the Hartree approximation was investigated in Ref. [1]. Here, we investigate the effects arising of RPA correlations in a multi-component system. As in Sec. II, we study the response in both nonrelativistic and relativistic modes of dense matter.

\section{A. Nonrelativistic Models}

We begin by considering a two-component system comprised of neutrons and protons. At first, we ignore the electromagnetic correlations between the protons and develop the basic formalism required to calculate the correlated response of the strongly interacting system to neutrino scattering. For a two-component system, the Hartree polarization function may be written as a $2 \times 2$ matrix, diagonal $\left(\Pi_{p}^{0}, \Pi_{n}^{0}\right)$, where $\Pi_{p}^{0}$ and $\Pi_{n}^{0}$ are the proton and neutron polarizations, whose real and imaginary parts may be explicitly evaluated using Eq. (3) and Eq. (7), respectively. To compute the $2 \times 2$ RPA polarization function, we parameterize the $\mathrm{p}-\mathrm{h}$ interactions in the spin independent and spin dependent channels. The spin independent interaction is given by $f_{n n}, f_{p p}$, and $f_{n p}$, and the spin dependent interaction by $g_{n n}, g_{p p}$, and $g_{n p}$. It must be borne in mind that these parameters are associated with in-medium $\mathrm{p}$-h interaction and not the particle-particle interactions. For example, the parameter $f_{n n}\left(g_{n n}\right)$ is a measure of the matrix element between two $n n^{-1}$ states with identical (opposite) spin projections, and $f_{n p}\left(g_{n p}\right)$ measures the matrix element between a $n n^{-1}$ and $p p^{-1}$ states with identical (opposite) spin projections [37]. In matrix form, the interactions in the spin independent and spin dependent channels are given by

$$
D_{V}=\left[\begin{array}{cc}
f_{p p} & f_{p n} \\
f_{p n} & f_{n n}
\end{array}\right] \quad \text { and } \quad D_{A}=\left[\begin{array}{ll}
g_{p p} & g_{p n} \\
g_{p n} & g_{n n}
\end{array}\right] \text {, }
$$

respectively. The Dyson equation for the vector polarization is

$$
\Pi_{V}^{R P A}=\Pi^{0}+\Pi^{R P A} D_{V} \Pi^{0} .
$$

The axial polarization is obtained by replacing $D_{V}$ in the above equation by $D_{A}$. Solving the matrix equations, we obtain

$$
\begin{aligned}
& \Pi_{V}^{R P A}=\frac{1}{\Delta_{V}}\left[\begin{array}{cc}
\left(1-f_{n n} \Pi_{n}^{0}\right) \Pi_{p}^{0} & f_{n p} \Pi_{p}^{0} \Pi_{n}^{0} \\
f_{p n} \Pi_{p}^{0} \Pi_{n}^{0} & \left(1-f_{p p} \Pi_{p}^{0}\right) \Pi_{n}^{0}
\end{array}\right] \\
& \Delta_{V}=\left[1-f_{n n} \Pi_{n}^{0}-f_{p p} \Pi_{p}^{0}+f_{p p} \Pi_{p}^{0} f_{n n} \Pi_{n}^{0}-f_{n p}{ }^{2} \Pi_{n}^{0} \Pi_{p}^{0}\right]
\end{aligned}
$$


for the vector part, and

$$
\begin{aligned}
\Pi_{A}^{R P A} & =\frac{1}{\Delta_{A}}\left[\begin{array}{cc}
\left(1-g_{n n} \Pi_{n}^{0}\right) \Pi_{p}^{0} & g_{n p} \Pi_{p}^{0} \Pi_{n}^{0} \\
g_{p n} \Pi_{p}^{0} \Pi_{n}^{0} & \left(1-g_{p p} \Pi_{p}^{0}\right) \Pi_{n}^{0}
\end{array}\right] \\
\Delta_{A} & =\left[\begin{array}{ll}
1-g_{n n} \Pi_{n}^{0}-g_{p p} \Pi_{p}^{0}+g_{p p} \Pi_{p}^{0} g_{n n} \Pi_{n}^{0}-g_{n p}{ }^{2} \Pi_{n}^{0} \Pi_{p}^{0}
\end{array}\right]
\end{aligned}
$$

for the axial part. Multiplying the above polarization matrices by the appropriate vector and axial vector couplings for the neutrons and protons, we arrive at the vector and axial vector response functions:

$$
\begin{aligned}
S_{V}\left(q_{0}, q\right) & =\frac{1}{1-\exp \left(-q_{0} / T\right)} \operatorname{Im} \Pi_{V}^{R P A} \\
\Pi_{V}^{R P A} & =\left[\frac{\left(c_{V}^{p}\right)^{2}\left(1-f_{n n} \Pi_{n}^{0}\right) \Pi_{p}^{0}+\left(c_{V}^{n}\right)^{2}\left(1-f_{p p} \Pi_{p}^{0}\right) \Pi_{n}^{0}+2 c_{V}^{p} c_{V}^{n} f_{n p} \Pi_{n}^{0} \Pi_{p}^{0}}{\Delta_{V}}\right] \\
S_{A}\left(q_{0}, q\right) & =\frac{1}{1-\exp \left(-q_{0} / T\right)} \operatorname{Im} \Pi_{A}^{R P A} \\
\Pi_{A}^{R P A} & =\left[\frac{\left(c_{A}^{p}\right)^{2}\left(1-g_{n n} \Pi_{n}^{0}\right) \Pi_{p}^{0}+\left(c_{A}^{n}\right)^{2}\left(1-g_{p p} \Pi_{p}^{0}\right) \Pi_{n}^{0}+2 c_{A}^{p} c_{A}^{n} g_{n p} \Pi_{n}^{0} \Pi_{p}^{0}}{\Delta_{A}}\right]
\end{aligned}
$$

respectively. In terms of these response functions, the differential cross sections per unit volume is given by

$$
\begin{aligned}
\frac{1}{V} \frac{d^{3} \sigma\left(E_{1}\right)}{d \Omega^{2} d q_{0}} & =\frac{G_{F}^{2}}{8 \pi^{3}} \quad E_{3}^{2} \quad\left[1-f_{\nu}\left(E_{3}\right)\right] \\
& \times\left[(1+\cos \theta) S_{V}\left(q_{0}, q\right)+(3-\cos \theta) S_{A}\left(q_{0}, q\right)\right]
\end{aligned}
$$

where the various kinematical labels appearing above are as in Eq. (11). Using Eq. (33), the neutrino cross sections may be computed if the $\mathrm{p}$-h interaction is specified. From the discussion of the previous section, it is clear that these interactions are model dependent and that uncertainties are large. Therefore, we explore different dense matter models to identify the generic trends.

\section{Particle-Hole Interactions in Nuclear Matter}

In contrast to neutron-rich matter, for which we have little empirical information, the $\mathrm{p}$-h interaction in nuclear matter may be related to empirical values of the Fermi-liquid parameters at nuclear saturation density. The $\mathrm{p}$-h interaction is written in terms of the Fermi-liquid parameters in Eq. (8). In isospin-symmetric matter, $f_{n n}=f_{p p}$ and $g_{n n}=g_{p p}$. This allows us to directly relate the interaction strengths to Fermi-liquid parameters [37]:

$$
f_{n n}=\frac{F_{0}+F_{0}^{\prime}}{N_{0}}, \quad f_{n p}=\frac{F_{0}-F_{0}^{\prime}}{N_{0}}, \quad g_{n n}=\frac{G_{0}+G_{0}^{\prime}}{N_{0}}, \quad g_{n p}=\frac{G_{0}-G_{0}^{\prime}}{N_{0}}
$$

where $N_{0}=2 M^{*} k_{f} / \pi^{2}$ is the density of states at the Fermi surface. Note that we have retained only the the $l=0$ (s-wave) terms, since we expect the momentum transfer in 
the p-h channel to be small for low energy neutrino scattering. However, the momentum dependent part of the interaction, which gives rise to the $l=1$ term, $F_{1}=3\left(1-M^{*} / M\right)$ is still important since it determines the nucleon effective mass and hence $N_{0}$.

From FLT, we know that the $l=0$ Fermi-liquid parameters are directly related to macroscopic observables. $F_{0}$ is related to the isoscalar incompressibility $K=9 \partial P / \partial n_{B}$, and $F_{0}^{\prime}$ is related to the nuclear symmetry energy $a_{4}=\left(n_{B} / 2\right) \partial^{2} \epsilon / \partial n_{3}^{2}$, where $n_{3}=n_{n}-n_{p}$ is the isospin density. Explicitly,

$$
F_{0}=\left(K / 6 E_{F}\right)-1 \quad \text { and } F_{0}^{\prime}=\left(3 a_{4} / E_{F}\right)-1,
$$

with $E_{F}=\left(k_{F}^{2} / 2 M^{*}\right)$ being the Fermi energy. Note that in extracting the p-h interaction potential from experimental observables such as $K$ and $a_{4}$, a consistent value of $M^{*}$ must be employed, since both $F_{0}$ and $F_{0}^{\prime}$ depend on $M^{*}$.

At nuclear saturation density, investigation of the monopole resonances in nuclei suggest that the isoscalar compressibility $K \cong 240 \pm 40 \mathrm{MeV}$ [38]. Information from neutron-rich nuclei and observed isovector giant dipole resonances in nuclei require that $a_{4} \cong 32 \pm 5 \mathrm{MeV}$, and empirical determinations of the nucleon effective mass from level density measurements in nuclei favor $M^{*} / M \cong 0.7 \pm 0.1$. For typical values of $K=240 \mathrm{MeV}, a_{4}=30 \mathrm{MeV}$, and $M^{*} / M=0.75$, the Fermi-liquid parameters are: $F_{0}=-0.18, F_{0}^{\prime}=0.83$, and $F_{1}=0.75$. The spin dependent parameter $G_{0}$, which is related to the experimentally observed isoscalar spinflip resonances is estimated to be small, $G_{0}=0.1 \pm 0.1$ [39.40]. In contrast, the parameter $G_{0}^{\prime}$, which is related to the isovector spin-flip (Giant Gamow-Teller) resonances in nuclei is empirically estimated to be large, $G_{0}^{\prime} \cong 1.5 \pm 0.2$ [21,39].

Using the empirical values for the Fermi-liquid parameters: $F_{0}=-0.18, F_{0}^{\prime}=0.83, G_{0}=$ $0, G_{0}^{\prime}=1.7$, and $M^{*} / M=0.75$, we find that the numerical values for the p-h interaction strengths in the various channels are:

$$
\begin{array}{ll}
f_{n n}=f_{p p}=1.7 \times 10^{-5} \mathrm{MeV}^{-2}, & f_{n p}=-2.7 \times 10^{-5} \mathrm{MeV}^{-2}, \\
g_{n n}=g_{p p}=4.5 \times 10^{-5} \mathrm{MeV}^{-2}, & g_{n p}=-4.5 \times 10^{-5} \mathrm{MeV}^{-2} .
\end{array}
$$

Using these values, the neutrino scattering differential cross sections in nuclear matter are shown in Fig. 11. The upper panels are for matter at zero temperature, where only the positive $q_{0}$ response exists. The suppression due to $M^{*}$ effects are important amounting to $\sim 50 \%$ reduction. Correlations, which are predominantly repulsive in nature due to the large empirical values for $G_{0}^{\prime}$ and $F_{0}^{\prime}$, result in significant further reductions. The RPA result indicates a factor of two reduction in the differential cross sections in the region $q_{0} / q \ll v_{F}$, where $v_{F}$ is the Fermi velocity. The presence of a collective state in the region $q_{0} / q \sim v_{F}$ enhances the cross sections in this region. This enhancement, however, is not significant enough to override the large suppression seen in the region where $q_{0} / q$ is small.

Integrating over the $q_{0}-q$ space, we obtain the total cross section per unit volume or equivalently the inverse collision mean free path. This is shown in Fig. 12. The left panels show the cross sections calculated by taking into account only effects due to $M^{*}$. The results shown are for different temperatures and for a neutrino energy $E_{\nu}=\pi T$. Right panels show the ratio $\lambda_{R P A} / \lambda_{M *}$. The resulting increase in $\lambda$ due to the presence of a repulsive p-h interaction is approximately a factor of 2.5 at low temperature and decreases with increasing temperature. 


\section{Density Dependence}

The density dependence of the Fermi-liquid parameters is poorly constrained by data. Although numerous theoretical models have been constructed to gain insight into their high density behavior, there appears to be no general consensus at the present time. Microscopic calculations of neutron matter differ quantitatively depending on their underlying assumptions. These model dependencies are so large that no generic qualitative trends may be identified. The exception is the isoscalar parameter $F_{0}$, which becomes positive and increases with increasing density, a feature which may be expected on general grounds as the repulsive vector meson contributions dominate. The uncertainties associated with $F_{0}^{\prime}$ are related to the model dependence of the nuclear symmetry energy. In models that favor a less than linear increase of $a_{4}$ with density, $F_{0}^{\prime}$ is expected to decrease with increasing density (see Eq. (35)). The state of the art microscopic many-body calculations favor a modest increase in the nuclear symmetry energy at intermediate densities [27].31]; thus, we may expect that $F_{0}^{\prime}$ will generally decrease. The parameter $G_{0}^{\prime}$ is related to pion condensation, since it is a measure the spin-isospin susceptibility of nuclear matter. The large repulsive character of $G_{0}^{\prime}$ strongly inhibits s-wave pion condensation in the vicinity of the nuclear saturation density. However, at higher densities pion condensation cannot be ruled out a priori 30,31. Thus, while we may expect $G_{0}^{\prime}$ to decrease somewhat with increasing density, quantitatively it remains very sensitive to the underlying model. The density dependence of the isoscalar spin parameter $G_{0}$, which is not well constrained even at nuclear density, is largely unknown.

Faced with these uncertainties, we begin by assuming that the spin dependent parameters are fixed at their empirical values (determined at saturation density), and use schematic models to explore the influence of the density dependence of $F_{0}$ and $F_{0}^{\prime}$. For this purpose, we employ a simple parametric form for the EOS 41] (see Appendix A). This model does not explicitly address the role of spin dependent interactions and assumes that the favored ground state is spin symmetric. In particular, we choose the Skyrme-like models labelled 'SLn2' with a linear increase in the nuclear symmetry energy. The index ' $\mathrm{n}$ ' in 'SLn2' takes on the values $n=1,2$ and 3 for which $K=120,180$ and $240 \mathrm{MeV}$, respectively. The magnitudes of the RPA corrections to the neutrino mean free paths for these different EOS models are shown in Fig. 13. Since the dominant contribution to the scattering cross section arises from the axial vector response function, the magnitude of the RPA corrections are mostly sensitive to the spin dependent parameters. Thus, although the vector response of the nuclear medium is modified by about 50-80 \% at high density due to RPA effects, the changes due to the varying stiffness of the dense matter EOS are small. This suggests that the neutrino mean free paths will not be significantly altered due to variations in the nuclear compressibility $\left(F_{0}\right)$ or due to variations in the nuclear symmetry energy $\left(F_{0}^{\prime}\right)$ as long as the axial contributions are not drastically reduced.

Fig. 14 shows the behavior of the neutrino mean free paths in symmetric nuclear matter for the EOS labelled SL22 as a function of density for the temperatures of interest. A comparison of the upper and lower panels show that there is significant temperature dependence in the Hartree and RPA results, and that RPA corrections decrease with increasing temperature.

To explore the sensitivity to density dependent spin interactions, we employ the Skyrme 
models discussed earlier. In Fig. 15 the Fermi-liquid parameters in symmetric matter are shown. As in the case of pure neutron matter, the results show a large model dependence. The top panels in Fig. 16 contain the neutrino cross sections for the model SGII, which has been constrained to fit the isovector giant dipole and Gamow-Teller resonances observed in nuclei. The neutrino cross sections are enhanced at high density due to an attractive interaction in the spin channels. Similar trends may also be expected for the model SkM*, since the Fermi-liquid parameters show similar qualitative trends. The models SLy4 and SLy5 show distinctly different behavior, especially in the $G_{0}$ and $G_{0}^{\prime}$ channels, in addition to favoring a larger symmetry energy. Neutrino mean free paths for the model SLy4 are shown in the lower panels of Fig. 16. This model is poorly behaved in the spin-isospin channel, the negative value for $G_{0}^{\prime}$ at nuclear density testifying to this fact. Unlike the model SGII, which is stable up to $\sim 3 n_{0}$ (for higher densities $G_{0}<-1$ ), the model SLy4 is stable in the spin channel for all densities. However, it becomes unstable to long wavelength spin-isospin oscillations (pion condensation) for $n>2 n_{0}$. It is this large attraction that is responsible for the significant reductions in the mean free path observed (see lower right panel Fig. 16).

We wish to emphasize that the above model predictions are strictly valid only for those densities at which the stability conditions, which require all the $\ell=0$ Fermi-liquid parameters be greater -1 , are satisfied. In view of this, the range of applicability of these models is limited to a small window in density centered around the saturation density (see Fig. 15). We have investigated a large class of Skyrme models and find this to be a generic feature. It is not clear at the present time if the choice of a better parameter set will help alleviate this feature. More likely, additional momentum and density dependent terms are required. Despite these shortcomings, the Skyrme energy density functional method provides a very simple and transparent scheme to calculate both the EOS and consistent opacities required for astrophysical problems. We hope that, in the future, a Skyrme parameterization that mimics the results of microscopic many-body calculations will become available so as to facilitate finite temperature calculations of dense matter at arbitrary asymmetry.

\section{2. $\beta$-Stable Asymmetric Matter}

We now study the response of charge neutral matter in $\beta$-equilibrium using the schematic model labelled SL22, and the Skyrme models SGII and SLy4. The composition of matter with and without neutrino trapping, and at finite temperature is calculated as outlined in Ref. [1]. The linear increase in the nuclear symmetry energy in the model SL22 favors relatively large proton fractions compared to those predicted by the SGII and SLy4. Using the neutron and proton chemical potentials obtained by solving the EOS, neutrino mean free paths are calculated as described earlier. The electrons, which are relativistic for all densities of interest, cannot be consistently accounted for in a nonrelativistic treatment. Therefore, we defer the discussion of the response which includes electrons to the next section.

At first, we ignore effects due to electromagnetic correlations between the charged components and consider only the response of the strongly interacting baryonic components. The differential cross sections are computed using Eq. (33) with the appropriate baryon chemical potentials and effective masses. The $\mathrm{p}$ - $\mathrm{h}$ interaction potentials in the spin independent channel are computed consistently with the schematic EOS as described earlier, i.e. 
by taking functional derivatives of the energy density with respect to neutron and proton number densities. In general, for asymmetric matter $f_{n n} \neq f_{p p}$. Therefore, we require three independent quantities to characterize the $\mathrm{p}$-h interaction in the spin independent channel (unlike only two that were required for nuclear matter). The three relevant quantities may be computed directly, if the form of the potential part of the single particle spectrum is known. For the model SL22 the single particle spectrum obtained by a functional differentiation of the potential energy density is given in Eq. (66) of Appendix A. The functional derivatives of the single particle potential energy are then related to the p-h interaction parameters as follows:

$$
f_{n n}=\frac{\delta U_{n}}{\delta n_{n}}, \quad f_{p p}=\frac{\delta U_{p}}{\delta n_{p}}, \quad \text { and } \quad f_{n p}=\frac{\delta U_{n}}{\delta n_{p}}=\frac{\delta U_{n}}{\delta n_{n}} .
$$

For the spin dependent part, we use empirical values since our schematic EOS does not explicitly contain spin dependent interactions. These empirical values are determined at saturation density and for symmetric nuclear matter. There is a priori no reason to expect that they will remain unchanged with increasing density and asymmetry. Our choice is therefore primarily motivated by its simplicity, and to a lesser extent by microscopic calculations that indicate that these parameters do not change appreciably with density [28]. Fig. 17 shows the differential cross sections in stellar matter with zero neutrino chemical potential. Results for $n_{B}=n_{0}$ (bottom panels) and for $n_{B}=3 n_{0}$ (top panels), with proton fractions $Y_{p}=0.049$ and $Y_{p}=0.22$, respectively, are shown. The structure seen in the Hartree results (dashed curves) at $T=0$ is associated with the fact that the single pair component of the the proton response is available only in the kinematical region where $q_{0} \leq|\vec{q}| v_{F}$, where $v_{F}=k_{F}^{p} / M_{p}^{*}$ is the velocity at the Fermi surface. For $n_{B}=n_{0}$, the RPA cross section is suppressed by roughly a factor of two. The choice of a large and repulsive spin interaction is somewhat compensated by the attractive isoscalar component of the $\mathrm{p}-\mathrm{h}$ interaction. For $n_{B}=3 n_{0}, \mathrm{p}$-h interactions are repulsive in all channels and account for the larger (factor of four) suppression seen in the upper panels of Fig. 17 .

In the Skyrme models, the p-h interaction in all the relevant channels is explicitly calculable as functions of density and proton fraction, and are given in Appendix A. The differential cross sections are computed using Eq. (33) with the appropriate model dependent inputs, i.e. the p-h interaction, the neutron and proton chemical potentials and the effective masses. Results for the models SGII and SLy4 are shown in Fig 18. As a result of the large density and asymmetry dependence of the p-h interaction predicted by Skyrme models, the RPA corrections vary significantly with changing density and proton fraction. The top panels show results for $n_{B}=0.16 \mathrm{fm}^{-3}$ and proton fraction $Y_{p}=0.05$. The differences seen in the Hartree results are due to the different effective masses and proton fractions predicted by these models. The RPA results differ qualitatively between these two models. At nuclear saturation density (left panels), both models are stable with respect to spin-isospin fluctuations and contain nearly equal proton fractions. The RPA response differs mainly due to the different spin-dependent interactions: SLy4 favors a large and attractive spin-isospin interaction, which strongly correlates the neutron and proton spins and accounts for the enhancement in the kinematical region where the protons contribute (region where $q_{0} / q \leq v_{F}^{p}$ ). In model SGII, the situation is reversed since the $\mathrm{p}$-h interaction is weakly repulsive in the spin channels and accounts for the modest suppression seen in the left-lower panel of Fig. 18. At $2 n_{0}$, the p-h interaction is large and attractive in both the 
isoscalar and isovector spin channels for both models. For model SLy4, this turns out to be so large that the spin-symmetric $\beta$-equilibrium ground state is unstable to long wavelength spin-isospin fluctuations, a feature which was already encountered in the case of symmetric matter. Although the spin forces are large and attractive even for the model SGII, they are not sufficiently large to energetically favor a spin-asymmetric state. The resulting enhancement in the RPA response is about a factor of 4 at small $q_{0}$ (note that for $\mathrm{T}=10 \mathrm{MeV}$, the RPA response plotted has been multiplied by 0.25 ) and decreases with increasing $q_{0}$.

The collision mean free paths are shown in Fig. 19. The Hartree mean free paths are shown in the left panels and the ratio of the RPA mean free paths to the Hartree results are shown in the right panels. The results show trends similar to those observed earlier for neutron matter and for symmetric nuclear matter. Results for the Skyrme models are not shown, since the RPA modes become unstable with increasing density and asymmetry. At low density and for small asymmetries, the trends are similar to those shown in Fig. 16.

\section{B. Relativistic Models}

The basic formalism required to calculate the response of a system containing nucleons and electrons within the framework of a relativistic mean field theory may be found in Ref. [8]. Here, we summarize the main results of their work and extend it to incorporate spin dependent correlations.

Neutron and proton single pair excitations are coupled due to strong interactions. In addition, the proton and electron $\mathrm{p}$-h excitations are coupled due to electromagnetic interactions. To incorporate these correlations consistently, we write the various polarizations functions in matrix form. The vector part of the polarization has transverse and longitudinal components. The transverse part is affected only due to the vector mesons. For a system comprised of neutrons, protons, and electrons, the RPA polarization is given by the Dyson equation

$$
\Pi_{T}^{R P A}=\Pi_{T}+\Pi_{T}^{R P A} D_{V} \Pi_{T}
$$

where the three-component transverse polarization matrix is diagonal $\left(\Pi_{T}^{e}, \Pi_{T}^{p}, \Pi_{T}^{n}\right)$ and the interaction matrix, defined in terms of $\chi_{\gamma}=-e^{2} / q_{\mu}^{2}, \chi_{V}=\chi_{\omega}+\chi_{\rho}$, and $\chi_{I}=\chi_{\omega}-\chi_{\rho}$, is given by

$$
D_{V}=\left[\begin{array}{ccc}
-\chi_{\gamma} & \chi_{\gamma} & 0 \\
\chi_{\gamma} & -\chi_{\gamma}-\chi_{V} & -\chi_{I} \\
0 & -\chi_{I} & -\chi_{V}
\end{array}\right]
$$

The above equation accounts for electromagnetic, isoscalar, and isovector strong interaction

correlations. Since the scalar $\sigma$ - and the vector $\omega$-mesons mix strongly in the longitudinal channel, the polarization matrix is written as a $4 \times 4$ matrix equation [45]. Explicitly, the Dyson equation for the longitudinal part is

$$
\Pi_{L}^{R P A}=\Pi_{L}+\Pi_{L}^{R P A} D_{L} \Pi_{L}
$$

with the longitudinal polarization for the $n, p$, and $e$ system given by 


$$
\Pi_{L}=\left[\begin{array}{cccc}
\Pi_{00}^{e} & 0 & 0 & 0 \\
0 & \Pi_{S}^{p}+\Pi_{S}^{n} & \Pi_{M}^{p} & \Pi_{M}^{n} \\
0 & \Pi_{M}^{p} & \Pi_{00}^{p} & 0 \\
0 & \Pi_{M}^{p} & 0 & \Pi_{00}^{p}
\end{array}\right]
$$

and the interaction matrix

$$
D_{L}=\left[\begin{array}{cccc}
-\tilde{\chi}_{\gamma} & 0 & \tilde{\chi}_{\gamma} & 0 \\
0 & -\chi_{S} & 0 & 0 \\
\tilde{\chi}_{\gamma} & 0 & -\tilde{\chi}_{\gamma}-\tilde{\chi}_{V} & -\tilde{\chi}_{I} \\
0 & 0 & -\tilde{\chi}_{I} & -\tilde{\chi}_{V}
\end{array}\right]
$$

The interaction parameters are: $\tilde{\chi}_{V}=q_{\mu}^{2} \chi_{V} / q^{2}, \tilde{\chi}_{I}=q_{\mu}^{2} \chi_{I} / q^{2}$, and $\tilde{\chi}_{\gamma}=q_{\mu}^{2} \chi_{\gamma} / q^{2}$. The dominant modification to the axial polarizations at small momentum transfers arises due to the pseudo-vector contact interaction discussed earlier and is parameterized by the Migdal parameter $g^{\prime}$. Further, since the electromagnetic contributions to spin correlations are negligible, we account only for the correlations due to strong interactions between neutrons and protons. It is conventional to introduce the short range correlations by modifying the pion propagator in the pseudo-vector form of the pion-nucleon interaction described by the Lagrangian density

$$
L=-\left(f_{\pi} / m_{\pi}\right) \bar{\psi} \gamma^{5} \gamma^{\mu} \tau \psi \partial_{\mu} \pi
$$

The Migdal parameter $g^{\prime}$ enters through the modified pion propagator

$$
\frac{q_{\mu} q_{\nu}}{q_{m} u^{2}-m_{\pi}^{2}+i \epsilon} \rightarrow \frac{q_{\mu} q_{\nu}}{q_{m} u^{2}-m_{\pi}^{2}+i \epsilon}-g^{\prime} g_{\mu \nu}
$$

and the $N N \pi$ vertex becomes

$$
\Gamma_{N N \pi}^{\mu}=\sqrt{2}\left(f_{\pi} / m_{\pi}\right) \gamma_{5} \gamma^{\mu}
$$

At small momentum transfers, the repulsive contact term overwhelms the attraction arising due to one-pion exchange. For this reason, we retain only the contribution from the $g^{\prime}$ term in the RPA equations. For a two-component system, this requires us to extend the Dyson equation for the axial vector part in Eq. (25) to an $8 \times 8$ matrix. However, it can be explicitly shown that for $c_{A}^{p}=-c_{A}^{n}$, the cross terms cancel and the RPA axial polarization for the n-p system closely resembles that for a one-component system, but with $\Pi_{A}=\Pi_{A}^{n}+\Pi_{A}^{p}$. This greatly simplifies the formalism required to incorporate spin dependent correlations. The RPA axial polarization is therefore once again given in terms of Eq. (25).

We have ignored effects due to mixing of the vector and axial correlations, since they are proportional to $\Pi_{V A}$, which is negligible at small momentum and energy transfers.

Substituting the vector and axial vector RPA polarizations for the correlated 3 component system into Eq. (10), the differential cross section for the npe system is

$$
\frac{1}{V} \frac{d^{3} \sigma}{d^{2} \Omega d E_{\nu}}=-\frac{G_{F}^{2}}{32 \pi^{3}} \frac{E_{3}}{E_{1}}\left[1-f_{3}\left(E_{3}\right)\right] \operatorname{Im}\left[\frac{L_{\alpha \beta} \Pi_{R P A}^{\alpha \beta}}{1-\exp \left(-q_{0} / T\right)}\right] .
$$


Utilizing the particle fractions, nucleon effective masses, and particle chemical potentials in $\beta$-equilibrated stellar matter, the neutrino cross sections and scattering mean free paths are straightforwardly computed using the above equation.

In the model labelled RHA, the effects arising due to vacuum loops are explicitly taken into account both at the level of the EOS and the response functions. The differential cross sections for this model are shown in Fig. 20 for the model GM3, wherein nonlinear scalar selfinteractions play an important role [46] (it acts to lower the compression modulus, increase the nucleon effective mass and make the "effective" $\sigma$-meson mass density dependent). Since the Migdal parameter $g^{\prime}$ plays a dominant role in determining the RPA response, results for different values of $g^{\prime}$ are shown. The modification to the vector response due to scalar, vector, and isovector mesons is large, but, it does not translate to large suppressions in the differential cross sections (top left panel of Fig. 20), since the response is dominated by the axial current coupling of the neutrinos. The total suppression remains very sensitive to $g^{\prime}$ for which empirical values are expected to be in the range $0.5-0.7$. RPA correlations suppress the neutrino scattering cross sections by typically a factor of $2-3$.

The RPA and Hartree mean free paths of thermal neutrinos for the model GM3 are compared in Fig. 21. Effects due to correlations increase with density and decrease with temperature. The decrease in the ratio $\lambda_{R P A} / \lambda_{H}$ (lower panels) at high density is due to the decreasing $M^{*}$. The pseudovector point-like coupling introduced through $g^{\prime}$ is sensitive to $M^{*}$ 36 and its effects decrease with increasing density. The results in the lower left panels indicate that the RPA correlations, even for a large $g^{\prime}$, will typically increase the mean free path by a factor $2-2.5$.

\section{CHARGED CURRENT NEUTRINO CROSS SECTIONS IN MULTI-COMPONENT MATTER}

The discussion thus far has focussed upon the role of correlation effects on the neutral current scattering reactions. While these reactions are the only source of opacity for the $\mu$ and $\tau$ neutrinos, the electron neutrino mean free path is for the most part dominated by the charged current reaction $\nu+n \rightarrow e^{-}+p$. The absorption reaction is kinematically different from scattering, since the energy and momentum transfers are not limited by the matter's temperature alone. The energy transfer is typically of order $\hat{\mu}=\mu_{n}-\mu_{p}$. In the extremely degenerate case, for which $T / \mu<<1$, and in neutrino-poor matter, final state Pauli-blocking due to electrons and momentum conservation restricts the available phase space. During the deleptonization epoch, the typical neutrino momenta are large $(\sim 100-200 \mathrm{MeV})$ and despite the mismatch of the neutron, proton, and electron Fermi momenta, momentum conservation is easily satisfied with the aid of the neutrino's momentum. This is no longer true in the late stages of the cooling phase, in which the neutrino energies are of order $k_{B} T$. Further, a finite neutrino chemical potential affects the relative displacement between the neutron and proton chemical potentials according to $\hat{\mu}=\mu_{e}-\mu_{\nu}$. 


\section{A. Non-Relativistic Models}

We begin by extending the formalism developed in Sec. III to incorporate effects due to correlations. The differential cross section per unit volume, for the absorption reaction in matter containing neutrons, protons, and electrons follows from Eq. (1), and is given by

$$
\begin{aligned}
\frac{1}{V} \frac{d^{3} \sigma\left(E_{1}\right)}{d \Omega^{2} d q_{0}} & =\frac{G_{F}^{2}}{8 \pi^{3}} \quad E_{3}^{2} \quad\left[1-f_{e}\left(E_{3}\right)\right] \\
& \times\left[g_{V}^{2}(1+\cos \theta) S_{V}^{\tau}\left(q_{0}, q\right)+g_{A}^{2}(3-\cos \theta) S_{A}^{\tau}\left(q_{0}, q\right)\right],
\end{aligned}
$$

where $g_{V}=1$ and $g_{A}=1.26$ are the charged current vector and axial vector coupling constants. The particle labels 2 and 4 correspond to the neutron and proton, respectively, and 3 to final state electron. The other kinematical variables are similar to the case of scattering. The isospin and spin-isospin density response functions are defined in terms of the asymmetric polarization $\Pi^{\tau}$. Due to the difference between neutron and proton chemical potentials, and of the single particle energies, $\Pi^{\tau}$ differs significantly from $\Pi^{0}$. For the simple form of the single particle energy given by Eq. (4), an analytic expression for the imaginary part may be derived (see Ref. [1] for details),

$$
\begin{aligned}
\operatorname{Im} \Pi^{\tau} & =\frac{M_{2}^{*} M_{4}^{*} T}{\pi q}\left[\xi_{-}-\xi_{+}\right] \\
\xi_{ \pm} & =\ln \left[\frac{1+\exp \left[\left(e_{ \pm}-\mu_{2}+U_{2}\right) / T\right]}{1+\exp \left[\left(e_{ \pm}+q_{0}-\mu_{4}+U_{2}\right) / T\right]}\right] \\
e_{ \pm} & =\frac{p_{ \pm}^{2}}{2 M_{2}^{*}} \quad p_{ \pm}^{2}=\frac{2 q^{2}}{\chi^{2}}\left[\left(1+\frac{\chi M_{4}^{*} c}{q^{2}}\right) \pm \sqrt{1+\frac{2 \chi M_{4}^{*} c}{q^{2}}}\right] .
\end{aligned}
$$

The factors

$$
\chi=1-\frac{M_{4}^{*}}{M_{2}^{*}} \quad \text { and } \quad c=q_{0}+U_{2}-U_{4}-\frac{q^{2}}{2 M_{4}^{*}},
$$

arise due to the isospin dependence of the single particle potential energies. The real part is calculated numerically by using the Kramers-Kronig relation Eq. (7). The RPA response functions are related to the Hartree polarization $\Pi^{\tau}$ by the Dyson equation. Explicitly,

$$
\begin{aligned}
& S_{V}^{\tau}\left(q_{0}, q\right)=\frac{1}{1-\exp \left[-\left(q_{0}+\left(\mu_{2}-\mu_{4}\right)\right) / T\right]} \operatorname{Im}\left[\frac{\Pi^{\tau}}{1-f_{c} \Pi^{\tau}}\right], \\
& S_{A}^{c}\left(q_{0}, q\right)=\frac{1}{1-\exp \left[-\left(q_{0}+\left(\mu_{2}-\mu_{4}\right)\right) / T\right]} \operatorname{Im}\left[\frac{\Pi^{\tau}}{1-g_{c} \Pi^{\tau}}\right],
\end{aligned}
$$

where the $\mathrm{p}$-h interaction in the vector and axial vector channels are given by $f_{c}$ and $g_{c}$, respectively.

\section{Symmetric Nuclear Matter}

To elucidate the role of correlations, we begin with a brief discussion of the neutrino absorption reactions in symmetric nuclear matter. Here, the kinematical complications 
arising due to dissimilar neutron and proton Fermi surfaces and electron blocking are absent. To begin, we once again need the strength of the p-h interaction for the spin independent and the spin dependent channels. In contrast to scattering, both charge or isospin are transferred along the $\mathrm{p}$ - $\mathrm{h}$ channel in the charged current absorption reaction. Thus, only the isospin changing part of the p-h interaction is relevant for the charged current reaction. In terms of the Fermi-liquid parameters, this implies that only $F_{0}^{\prime}$ and $G_{0}^{\prime}$ contribute. For zero range interactions, the potentials required to calculate the RPA response are given by $f_{c}=2 F_{0}^{\prime} / N_{0}$ and $g_{c}=2 G_{0}^{\prime} / N_{0}$, where the factor two arises due to isospin considerations. In Fig. 22, the differential cross section for the charged current neutrino absorption reaction in symmetric nuclear matter is shown. As discussed earlier, large and positive empirical Fermi-liquid parameters indicate a correspondingly large and repulsive $\mathrm{p}$-h interaction. This accounts for the large suppression seen at small $q_{0}$. The suppression is roughly proportional to $\left(1+F_{0}^{\prime}\right)^{-2}$ in the vector response and $\left(1+G_{0}^{\prime}\right)^{-2}$ in the axial response. At high $q_{0}$ and for small temperature, well defined collective modes in the isospin-density and spin-isospin density channels appear. These correspond to the well known Giant Dipole (labelled as GD in Fig. 22) and Giant Gamow-Teller (labelled as GT) resonances found in nuclei. With increasing temperature, these collective states are significantly broadened due to Landau damping (see the right panel of Fig. 22).

\section{Asymmetric Nuclear Matter}

In asymmetric nuclear matter, strong interactions affect the charged current opacity both because of correlations as discussed above, and because of the important role they play in determining the quantity $\hat{\mu}=\mu_{n}-\mu_{p}$. The latter, was discussed earlier in Ref. [1]. In the following discussion, we show that the $\mathrm{p}$-h interaction in the vector channel and $\hat{\mu}$ are directly related. It was noted in Eq. (35) that $F_{0}^{\prime}$ was related to the nuclear symmetry energy. The nuclear symmetry energy in turn determines $\hat{\mu}$, and thereby the proton fraction for stellar matter in $\beta$-equilibrium [41]. Thus, a particular choice of the $\mathrm{p}$-h interaction in the isospin-density channel uniquely determines both $\hat{\mu}$ and the extent to which correlations suppress the cross sections. Relative to the case in which no interactions are considered, these effects oppose each other. From Eq. (35), it may be verified that $\hat{\mu}$ is linearly proportional to $\left(1+F_{0}^{\prime}\right)$. Correlations suppress the isospin density fluctuations by a factor roughly proportional to $\left(1+F_{0}^{\prime}\right)^{-2}$. The cumulative effect on the total absorption cross section is easily deduced by noting that the cross section is a linear function of $\hat{\mu}$ (see Ref. [1]), and is inversely proportional to $\left(1+F_{0}^{\prime}\right)^{2}$. This underscores the need to employ a p-h interaction that is consistent with the EOS employed to compute the composition of charge neutral $\beta$-equilibrated matter. Here, we employ the schematic potential model SL22 to compute the composition and the $\mathrm{p}$-h interaction in the spin independent channel.

The $\mathrm{p}$-h interaction in the spin-isospin channel has been studied extensively in the context of charge-exchange nuclear reactions [39], muon capture rates on nuclei [42], and pion condensation [43]. In these studies, a p-h interaction arising due to the exchange of $\pi$ - and $\rho$-mesons are included to account for the momentum dependence, in addition to the short range repulsion parametrized through the Migdal parameter $g^{\prime}$. The isovector interaction in the longitudinal channel arises due to $\pi$ exchange and in the transverse channel due to $\rho$-meson exchange. We employ this widely-used form for the $\mathrm{p}$-h interaction, which has been 
successful in describing a variety of nuclear phenomena 44]. The longitudinal and transverse potentials in the $\pi+\rho+g^{\prime}$ model are given by

$$
\begin{aligned}
& V_{L}\left(q_{0}, q\right)=\frac{f_{\pi}^{2}}{m_{\pi}^{2}}\left(\frac{\mathbf{q}^{2}}{q_{0}^{2}-\mathbf{q}^{2}-m_{\pi}^{2}} F_{\pi}^{2}(q)+g^{\prime}\right) \\
& V_{T}\left(q_{0}, q\right)=\frac{f_{\pi}^{2}}{m_{\pi}^{2}}\left(\frac{\mathbf{q}^{2} C_{\rho}}{q_{0}^{2}-\mathbf{q}^{2}-m_{\rho}^{2}} F_{\rho}^{2}(q)+g^{\prime}\right),
\end{aligned}
$$

where $C_{\rho}=2, F_{\pi}=\left(\Lambda^{2}-m_{\pi}^{2}\right) /\left(\Lambda^{2}-q^{2}\right)$ and $F_{\rho}=\left(\Lambda_{\rho}^{2}-m_{\rho}^{2}\right) /\left(\Lambda_{\rho}^{2}-q^{2}\right)$ are the $\pi N N$ and $\rho N N$ form factors [44, and the numerical value of $g^{\prime}$ used was 0.6. With this choice for the $\mathrm{p}-\mathrm{h}$ interaction, the axial response function is given by

$$
\begin{aligned}
S_{A}^{c}\left(q_{0}, q\right) & =\left[\frac{1}{1-\exp \left[-\left(q_{0}+\hat{\mu}\right) / T\right]}\right] \operatorname{Im} \Pi^{\tau}\left(q_{o}, q\right)\left(\frac{1}{3 \epsilon_{L}}+\frac{2}{3 \epsilon_{T}}\right) \\
\epsilon_{L} & =\left[1-2 V_{L} \operatorname{Re} \Pi^{0}\left(q_{o}, q\right)\right]^{2}+\left[2 V_{L} \operatorname{Im} \Pi^{0}\left(q_{o}, q\right)\right]^{2}, \\
\epsilon_{T} & =\left[1-2 V_{L} \operatorname{Re} \Pi^{0}\left(q_{o}, q\right)\right]^{2}+\left[2 V_{T} \operatorname{Im} \Pi^{0}\left(q_{o}, q\right)\right]^{2} .
\end{aligned}
$$

Using the axial response function given above and a $\mathrm{p}$-h interaction in the isospin-density channel, which is directly related to the nuclear symmetry energy predicted by the EOS, the neutrino absorption cross sections are computed.

In Fig. 23, the Hartree and RPA results for the differential cross section for the absorption reaction are shown for $n_{B}=0.16 \mathrm{fm}^{-3}$ and $n_{B}=0.48 \mathrm{fm}^{-3}$. The neutrino chemical potential and proton fraction determined by the finite temperature EOS are also shown in the figure. Since only those neutrinos close to the Fermi surface participate in transport, we have chosen the neutrino energy $E_{\nu}=\mu_{\nu}$. The response peaks in the region where $q_{0} \sim-\hat{\mu}$. The RPA suppression is roughly a factor of two for the kinematics shown here. The relatively smaller suppression seen here is due to the large energy and momentum transferred to the baryons. Since the charged current probes small distances, the effects of many-body correlations are somewhat suppressed. Further, the pion exchange contribution to the $\mathrm{p}$ - $\mathrm{h}$ interaction is attractive at large $\vec{q}$ and acts to decrease the short range repulsion due to $g^{\prime}$.

The neutrino absorption mean free path is shown in Fig. 24 for neutrino trapped matter at $Y_{L}=0.4$ for different temperatures and densities of relevance. The trends are very similar to the case of scattering. The left panel shows the Hartree results, wherein effects arising due to the density dependent nucleon effective masses and single particle potentials are included. The increase in the mean free path at high density arises due to the rapidly dropping $M^{*}\left(n_{B}\right)$ and the use of a nonrelativistic form for the single particle energy. The magnitude of the RPA corrections are shown in the right panels. On average, the enhancement is about a factor $2-3$. At intermediate baryon density, this enhancement increases with density and decreases with temperature. At higher density, the rapid decrease in $M^{*}$ decreases the density of $\mathrm{p}$-h states at the Fermi surface and thereby reduces the magnitude of the RPA corrections.

During the cooling epoch of a protoneutron star, the neutrino chemical potential becomes progressively smaller. For typical electron neutrino energies of order $T$, the kinematical restriction on the charged current process becomes important at low temperature. The density dependence of the symmetry energy essentially determines the density and temperature at which these kinematical restrictions severely inhibit the charged current rates. In the model 
SL22, which is characterized by a linear symmetry energy, the kinematical restrictions are unimportant for $n_{B} \geq 2$. At lower densities, and for $T \ll \mu_{p}$, there is significant suppression.

In Fig. 25, the Hartree and RPA differential cross sections are compared. The momentum transfer was held constant at $|\vec{q}|=\mu_{e}+\pi T$, to ensure momentum conservation. The response peaks in the region $q_{0} \sim\left(-\mu_{e}+E_{\nu}\right)$ and exponentially decreases for larger $q_{0}$ due to Pauliblocking of the degenerate electrons. On the other hand, the final state Pauli-blocking due to protons is negligible, since protons are fairly nondegenerate. This, combined with the large negative energy transfers, results in the real part $\Pi^{\tau}$ becoming large and negative, leading to significant reductions (factor $\sim 4$ ) in the RPA cross sections. For $n_{B}=0.48$ $\mathrm{fm}^{-3}$, a larger proton fraction and a lower nucleon effective mass decreases the magnitude of the real part $\Pi^{\tau}$. In this case, the suppression due to correlations is only about a factor of $1.5-2$.

In Fig. 26, neutrino absorption mean free paths for matter in which $\mu_{\nu}=0$ are shown. The trend identified in the preceding discussion, by which we may expect to find a large enhancement at intermediate densities and low temperatures, is clearly seen. For $n_{B} \geq 2 n_{0}$, correlations enhance the mean free paths by a factor $\sim 3-4$. For intermediate densities, the enhancement is significantly larger and very sensitive to the ambient temperature.

\section{B. Relativistic Models}

In field theoretical models, isovector correlations are induced by interactions mediated by the $\rho$-meson. Axial vector correlations, in the spin-isospin channel, arise due to the presence of a strong short ranged component, which is commonly parameterized as a contact interaction of strength $g^{\prime}$. The calculation of these vector and axial vector responses is similar to that discussed in Sec. IIB, but with two important differences. First, the $\sigma$ and $\omega$-mesons do not contribute when isospin is transferred along the p-h channel. Second, the polarization functions now describe the propagation of neutron-hole and proton-particle states, which depend on $\hat{\mu}=\mu_{n}-\mu_{p}$ and the isovector part of the single particle potential energy. The relevant polarizations are collected in Appendix B.

The differential cross section for the charged current absorption reaction is the same as that for neutral current scattering, Eq. (10), with the modification that $f_{3}\left(E_{3}\right)$ is replaced by $f_{e}\left(E_{e}\right)$. The vector part of the correlation function $\Pi_{\alpha \beta}^{R}$ is modified due to the $\rho$-meson contribution. Explicitly, the correction to the longitudinal part is

$$
\delta \Pi_{00}^{V}=\frac{-2 \tilde{\chi}_{I} \Pi_{00}^{2}}{1+2 \tilde{\chi}_{I} \Pi_{00}}
$$

and to the transverse part is

$$
\delta \Pi_{T}=\delta \Pi_{22}=\frac{2 \Pi_{T} \chi_{I} \Pi_{T}}{1+2 \chi_{I} \Pi_{T}} .
$$

For momentum transfers of interest, the axial vector current correlation function is modified mainly by the large and repulsive contact interaction of strength $\mathrm{g}^{\prime}$. As discussed earlier, this is conventionally introduced as a correction to the pion propogator (see Eq. (24)). In

principle, the contact term will lead to $\rho-\pi$ mixing via in-medium particle-hole states. These 
mixing effects are ignored in this work. This is justified both because $g^{\prime}$ is a phenomenological parameter, which is determined by ignoring these mixing effects, and because the mixing, which is proportional to the vector-axial vector polarization function, is negligible at small momentum transfers. The RPA equation for the axial vector polarization is as in Eq. (25), but with $\chi_{A}$ replaced by $2 \chi_{A}$, where the factor 2 arises from isospin considerations.

In Fig. 27, the RPA and Hartree differential cross sections for neutrino absorption are shown for $n_{B}=n_{0}$ and $n_{B}=3 n_{0}$, respectively. The results are presented for lepton rich, neutrino trapped matter with $Y_{L}=0.4$. The electron neutrino energy and the momentum transfer are both set equal to the neutrino chemical potential. The composition, individual chemical potentials, and the density dependent baryon effective masses are computed using the field theoretical model GM3. The RPA differential cross sections are significantly suppressed relative to the Hartree results, particularly around the peak position i.e., when $q_{0} \approx-\left(\mu_{n}-\mu_{p}\right)$. The suppression decreases with increasing density.

The absolute magnitude of the differential cross section depends sensitively on the neutrino energy, the electron chemical potential, composition, and temperature. In order to assess the overall effects of RPA correlations, both as a function of density and temperature, we integrate over $q_{0}$ and $q$ and compare the RPA and Hartree results for the neutrino mean free paths in neutrino-trapped matter in Fig. 28. As expected, RPA correlations increase the neutrino mean free paths. The trends are very similar to those seen in the nonrelativistic models. This is mainly due to the fact that $g^{\prime}$ plays a dominant role in suppressing the axial vector response in both cases. At low density, the minor differences seen between the two approaches may be attributed to the different p-h interactions employed. Relativistic effects become important with increasing density and this accounts for the differences seen at higher density.

The RPA and Hartree differential cross sections in neutrino poor matter $\left(\mu_{\nu}=0\right)$ are shown in Fig. 29. Due to important compositional and kinematical differences, the neutrino absorption cross sections differ from those of nonrelativistic models. At low density, Pauli blocking due to protons is small due to their low concentrations, which acts to enhance the polarization of the medium in the isospin channel. The suppression at $n_{B}=n_{0}$ (left panel) is approximately a factor of 4 . The suppression is reduced at high density; at $n_{B}=3 n_{0}$ (right panel), the suppression is approximately a factor of 2 .

The RPA and Hartree absorption mean free paths are compared in Fig. 30 as a function of density and temperature. The qualitative trends are again very similar to those encountered in the nonrelativistic case (see Fig. 26). The quantitative differences are due to the different compositions arising due to the different isovector interactions employed. It is clear that relativistic effects play an increasingly important role with increasing density, decreasing the level of suppression compared with the non-relativistic case.

\section{EFFECTS ON PROTONEUTRON STAR COOLING}

Within a tenth of a second of the core bounce which precedes a supernova explosion in the death of a massive star, the newly-formed neutron star, or protoneutron star (PNS), becomes quasistatic since its structure further evolves only in response to thermal and compositional

changes in its interior. These changes are, for the most part, controlled by the diffusion of neutrinos from the interior. At high density and for temperatures above several $\mathrm{MeV}$, the 
neutrino-matter reaction rates are sufficiently high to ensure that neutrinos are in thermal and chemical equilibrium. Thus, in the equilibrium diffusion aproximation (EDA), the neutrino distribution function in these regions is both nearly Fermi-Dirac and isotropic. These assumptions have been used to study the evolution of the PNS 47 49].

In the EDA, the distribution function in a spherically symmetric background can be approximated by an expansion in terms of Legendre polynomials to $O(\mu)$, with $\mu$ being the angle between the neutrino momentum and the radial direction. Explicitly,

$$
f(\omega, \mu)=f_{0}(\omega)+\mu f_{1}(\omega), \quad f_{0}=\left(1+\exp \left[\left(\omega-\mu_{\nu}\right) / T\right]\right)^{-1},
$$

where $f_{0}$ is the Fermi-Dirac distribution function at equilibrium $\left(T=T_{m a t}, \mu_{\nu}=\mu_{\nu}^{e q}\right)$, and $\omega$ and $\mu_{\nu}$ are the neutrino energy and chemical potential, respectively. This allows the energyintegrated fluxes of lepton number and energy to be expressed as a linear combination of gradients of the degeneracy parameter $\eta=\mu_{\nu} / T$ and the temperature [49]:

$$
\begin{aligned}
& F_{n}=-\frac{e^{-\Lambda} e^{-\phi} T^{2}}{6 \pi^{2}}\left[D_{3} \frac{\partial\left(T e^{\phi}\right)}{\partial r}+\left(T e^{\phi}\right) D_{2} \frac{\partial \eta}{\partial r}\right] \\
& F_{e}=-\frac{e^{-\Lambda} e^{-\phi} T^{3}}{6 \pi^{2}}\left[D_{4} \frac{\partial\left(T e^{\phi}\right)}{\partial r}+\left(T e^{\phi}\right) D_{3} \frac{\partial \eta}{\partial r}\right] .
\end{aligned}
$$

The diffusion coefficients $D_{2}, D_{3}$, and $D_{4}$ arise naturally from the transport equations and contain all the microphysics of the neutrino-matter interactions. They are defined by

$$
D_{n}=\int_{0}^{\infty} d x x^{n} D(\omega) f_{0}(\omega)\left(1-f_{0}(\omega)\right)
$$

where $x=\omega / T$ and $D(\omega)=\left(\kappa_{a}+\kappa_{s}\right)^{-1}$. The latter term includes the contribution of both absorption-emission opacities $\left(\kappa_{a}\right)$ and scattering opacities $\left(\kappa_{s}\right)$, which are related to the relevant cross sections by

$$
\kappa_{a, s}(\omega)=\frac{1}{1-f_{0}(\omega)} \frac{\sigma_{a, s}}{V} .
$$

The fluxes in Eqs. (65) and (66) are technically for one particle species. To include all six neutrino types, we redefine them in an obvious notation as

$$
D_{2}=D_{2}^{\nu_{e}}+D_{2}^{\bar{\nu}_{e}}, \quad D_{3}=D_{3}^{\nu_{e}}-D_{3}^{\bar{\nu}_{e}}, \quad D_{4}=D_{4}^{\nu_{e}}+D_{4}^{\bar{\nu}_{e}}+4 D_{4}^{\nu_{\mu}} .
$$

Effects on PNS evolution produced by changes in the neutrino opacities can thus be understood in terms of changes to the diffusion coefficients.

In Fig. 31 we show the ratio of each of the diffusion coefficients calculated in the RPA approximation to those calculated in the Hartree approximation. The left panels display the results for neutrino-free matter and the right panels show the results for neutrino-rich matter $\left(Y_{\nu}=0.06\right)$. In the region of interest during the first, or deleptonization, phase of the PNS evolution, in which the trapped neutrino abundances are high and $10 \mathrm{MeV}$ $<T<50 \mathrm{MeV}$, the RPA corrections to the diffusion coefficients are in the range 1.31.75 for matter above nuclear density. This correction, at first glance, seems smaller than expected; a correction factor $\approx 2$ was obtained for the mean free paths at $E_{\nu}=\pi \mathrm{T}$ (see 
Fig 21). However, the RPA correction redistributes strength in the spectrum (see Fig. 20), decreasing the cross section at energies near the peak while increasing the cross section for larger energy exchange. The diffusion coefficients result from an energy integration over the entire spectrum. Another important feature is that in the region below about half nuclear density, and at low temperatures, the RPA corrections are diminished since the composition of matter becomes dominated by nuclei instead of the npe liquid. Therefore, the diffusion coefficients in the outer regions of the PNS will not be strongly altered by RPA corrections.

During the second, or cooling, phase of PNS evolution, in which the excess neutrinos have been largely lost, the neutrino chemical potential is relatively low and nearly constant throughout the star. The evolution is then governed by the neutrino energy flux, Eq. (62), in which the electron neutrino chemical potential gradient can be ignored. Thus, $D_{4}$ will become the dominant diffusion coefficient. The temperatures are now smaller than in the earlier, deleptonization phase, being in the range $1<T / \mathrm{MeV}<20$. Fig. 31 shows that the RPA corrections, at three times nuclear density $\left(0.5 \mathrm{fm}^{-3}\right)$, are larger than in neutrinorich matter, and can exceed a factor of 2.5. Nevertheless, at and below nuclear density, the corrections are considerably smaller, as observed in the previous case. The effect of RPA corrections may be expected to be more evident in the cooling phase than in the deleptonization phase.

To study the effect in a realistic PNS simulation, we approximated the functions $D_{i}^{R P A} / D_{i}^{H}$, for $i=2-4$, by a fit in terms of $n_{B}, T$ and $Y_{\nu}$. Fig. 32 shows the function assumed for $Y_{\nu}=0$; the fits for other values of $Y_{\nu}$ are similar. Notice the strong density dependence in the region $n_{B}<n_{0}$. We used these fits to correct the diffusion coefficients calculated in Ref. [49], and performed a new PNS cooling simulation with the same code. We also wished to compare these results to those of Burrows and Sawyer [12], who also studied the effects of correlations in the RPA approximation but simply assumed that the total opacities were decreased by a factor of 3.33 above either $1 / 2.7$ or $1 / 5.4$ times nuclear density, respectively. Therefore, we performed two additional exploratory simulations with the identical assumptions. The results of all three simulations are displayed in Figs. 33 - 35.

In Fig. 33, the evolution of the central values of the important thermodynamic quantities is shown. The solid line corresponds to the baseline model, the dashed and dot-dashed lines to the exploratory models A and B, and the dotted line to our RPA calculation. The main effects of the larger mean free paths produced by the exploratory models and by our RPA corrections are that the inner core deleptonizes more quickly. In turn, the maxima in central temperature and entropy are reached on shorter timescales. In addition, the faster increase in thermal pressure in the core slows the compression associated with the deleptonization stage, although after $10 \mathrm{~s}$ the net compressions of all models converge.

The relatively large, early, changes in the central thermodynamic variables do not, however, translate into similarly large effects on observables such as the total neutrino luminosity and the average radiated neutrino energy, relative to the baseline simulation. The luminosities for the different models are shown as a function of time in Fig. 34 and Fig. 35. Figure 34 shows the early time development in detail. The exploratory models agree with the results reported by Burrows and Sawyer [12]. However, the magnitude of the effects when our full RPA corrections are applied is somewhat reduced compared to the exploratory models. It is especially important that at and below nuclear density, the corrections due to correlations are relatively small. Since information from the inner core is transmitted only by the 
neutrinos, the time scale to propagate any high density effect to the neutrinosphere is the neutrino diffusion time scale. Since the neutrinosphere is at a density approximately 1/100 of nuclear density, and large correlation corrections occur only above $1 / 3$ nuclear density where nuclei disappear, we find that correlation corrections calculated here have an effect at the neutrinosphere only after $1.5 \mathrm{~s}$. Moreover, the RPA suppresion we have calculated is considerably smaller than those reported in Ref. [12], reaching a maximum of about 30\% after $5 \mathrm{~s}$, compared to a luminosity increase of $50 \%$ after only $2 \mathrm{~s}$. However, the corrections are still very important during the longer-term cooling stage (see Fig. 35), and result in a more rapid onset of neutrino transparency compared to the Hartree results.

\section{CONCLUSIONS AND OUTLOOK}

The effects due to correlations are important, albeit model dependent. Due to the large neutrino-baryon axial vector coupling, the residual interaction in the spin dependent channels plays a crucial role in determining the magnitude of the RPA corrections. In pure neutron matter, these model dependencies were carefully studied. In the Skyrme model, the $\mathrm{p}$-h interaction in the spin channel becomes large and attractive with increasing density. This drastically reduces the neutrino mean free path by enhancing the spin response. The opposite behavior is suggested by variational calculations of the Fermi-liquid parameters in neutron matter. In this case, the residual interaction at high density is strongly repulsive in the spin channels. A repulsive p-h interaction generically shifts the strength from regions of small energy transfer $q_{0}$ to large energy transfer. In the case of strong repulsion, collective spin excitations arise and the strength at small $q_{0}$ is highly suppressed.

Not unexpectedly, the Skyrme models, in which the force parameters are calibrated only at nuclear density, either become unstable to spin fluctuations or violate causality at high density. Schematic models such as SL22, which are partly based on the Skyrme approach, are somewhat better behaved at high density, but they do not address the issue of spin dependence in the nucleon-nucleon interaction. In these models, the RPA corrections to the vector response may be calculated consistently with the EOS. For the axial part, an empirical spin-isospin interaction determined by the value of the Fermi-liquid parameter $\mathrm{G}_{0}^{\prime}$ in symmetric nuclear matter may be used.

The situation is quite similar in field-theoretical models, wherein the full relativistic structure of the baryon currents and more importantly, relativistic kinematics of the scattering reactions are naturally incorporated. In these models, the scalar-attractive contribution dominates at low density leading to enhancement in the vector part of the scattering response. With increasing density, the scalar decouples and the repulsive vector mesons suppress the cross sections. This is a generic feature. The RPA corrections in the vector channels generally suppress the neutrino cross sections at high density and moderately enhance it at low density. In addition, the RPA corrections in the vector channel and the EOS properties such as the compressibility and nuclear symmetry energy are intimately related. While this stresses the need for consistency between the EOS and neutrino opacities, there appears to be some degree of decoupling between the strong interaction effects that influence the EOS and those that influence the neutrino opacities. This is almost entirely due to the different roles played by a repulsive spin dependent interaction in EOS and opacity calculations. They directly influence the dominant part of the neutrino opacities but 
play little role in determining the properties of spin symmetric matter as long as the spin interaction is repulsive and the spin correlation energy is negligible compared to the other interaction energies. This is true for the field-theoretical model, wherein the introduction of a strong repulsive pseudovector-isovector interaction through the Migdal parameter does not contribute to the EOS at the mean field level, but plays an important role in determining the neutrino mean free path.

The RPA correlations in multi-component matter are qualitatively different from those in single-component system. The presence of even a small proton fraction affects the shape of the matters response function. The generic trend is that it increases the strength at small energy transfer. The inclusion of electromagnetic correlations does not significantly affect the neutrino mean free paths. The mixing of the photon with the vector mesons via the $\mathrm{p}-\mathrm{h}$ states could result in the presence of plasmon like collective states in the system. Although this might play an important role in determining the average energy transfer in neutrino matter interactions, it does not affect the overall neutrino mean free paths.

The dominant role played by spin dependent nucleon-nucleon interactions in the medium and its present poorly constrained status does not allow us to draw definite conclusions. For the vector part, we showed that RPA opacities are consistent with the underlying EOS. In subsequent works, we hope that better constraints on the spin dependent interactions in dense matter will allow calculations which will pin down its effects on both the EOS and on the opacities. At the present time, however, the RPA correlations remain sensitive to this poorly known density and isospin dependence of the $\mathrm{p}-\mathrm{h}$ spin interaction.

Notwithstanding the above caveats, and the differences between nonrelativistic and relativistic approaches such as the spin- and isospin-dependent interactions and the nucleon effective masses, suppressions of order 2-3, relative to the case in which correlations are ignored, are obtained. It is also satisfying that the independent investigations of Burrows and Sawyer 12 have led to a similar conclusion.

We studied the effects of RPA correlations on the evolution of protoneutron stars. Our calculations indicate that while RPA correlations suppress the neutrino cross section by a factor $\approx 3$ at thermal neutrino energies, the net effect on energy-integrated quantities such as neutrino diffusion coefficients is more moderate. Moreover, the effects of correlations are diminished below nuclear densities, and disappear in low-density matter that is dominated by nuclei. Thus, we observe significant effects in observable quantities such as neutrino luminosities only after 1 second, and it is only after several seconds that the neutrino luminosities are increased by as much as 30\%. Larger effects occur during the late-time thermal cooling phase, and the protoneutron star will become transparent on shorter timescales when RPA corrections are included.

\section{ACKNOWLEDGEMENTS}

We thank Adam Burrows, Chuck Horowitz, and Ray Sawyer for many beneficial discussions. This work was supported in part by the U.S. Department of Energy under Contracts DOE/DE-FG02-88ER-40388 and DOE/DE-FG02-87ER-40317 and by the NASA grant NAG52863. J. Pons gratefully acknowledges research support from the Spanish DGCYT grant PB97-1432. 


\section{APPENDIX A: PARTICLE-HOLE INTERACTIONS}

The p-h interaction in the Skyrme and Skyrme-like schematic models may be directly obtained by double functional differentiation of the potential energy density. The potential energy density for the density and temperatures of interest is mainly a function of baryon density and proton fraction. The temperature dependence, which enters only through the explicitly momentum dependent interactions, is weak. In what follows, we provide analytical expressions for the $\mathrm{p}$-h interaction at zero temperature.

The effective nucleon-nucleon interaction in the standard Skyrme model [23] is given in Eq. (9). The potential energy density in the Hartree-Fock approximation for the above interaction may be computed using the standard method by employing plane wave states for the nucleons [23]. In terms of the neutron and proton Fermi momenta $k_{F}^{n}$ and $k_{F}^{p}$, the neutron and proton densities $n_{n}$ and $n_{p}$, and the total baryon density $n=n_{n}+n_{p}$, the potential energy density is given by

$$
\begin{aligned}
\frac{1}{\Omega}\langle V\rangle & =\frac{1}{2} t_{0}\left(1+\frac{1}{2} x_{0}\right) n^{2}+\frac{1}{12} t_{3} n^{\gamma+2}\left(1+\frac{1}{2} x_{3}\right) \\
& +\frac{3}{20}\left[t_{1}\left(1+\frac{1}{2} x_{1}\right)+t_{2}\left(1+\frac{1}{2} x_{2}\right)\right] n^{2}\left(3 \pi^{2} n / 2\right)^{4 / 3} \\
& -\frac{1}{2} t_{0}\left(\frac{1}{2}+x_{0}\right)\left(n_{p}^{2}+n_{n}^{2}\right)-\frac{1}{12} t_{3} n^{\gamma}\left(\frac{1}{2}+x_{3}\right)\left(n_{p}^{2}+n_{n}^{2}\right) \\
& +\frac{3}{20}\left[-t_{1}\left(\frac{1}{2}+x_{1}\right)+t_{2}\left(\frac{1}{2}+x_{2}\right)\right]\left[n_{p}^{2} k_{F}^{2}(p)+n_{n}^{2} k_{F}^{2}(n)\right] .
\end{aligned}
$$

The single particle potential energy for a given nucleon with isospin index $\tau$ and with momentum $k$ is

$$
U_{\tau}(k)=\frac{\delta\langle V\rangle}{\delta n_{k k}},
$$

where $n_{k k}$ is the $(\tau \tau)$-diagonal element of the occupation number matrix

$$
n_{i j} \equiv\left\langle\Psi\left|a_{j}^{+} a_{i}\right| \Psi\right\rangle
$$

each label $i, j$ denoting momentum, spin, and isospin. For Skyrme interactions, one gets

$$
\begin{aligned}
U_{\tau}(k)= & t_{0}\left(1+\frac{1}{2} x_{0}\right) n+\frac{1}{6} t_{3} n^{\gamma}\left(1+\frac{1}{2} x_{3}\right) n \\
& +\frac{1}{12} \gamma t_{3} n^{\gamma-1}\left[\left(1+\frac{1}{2} x_{3}\right) n^{2}-\left(\frac{1}{2}+x_{3}\right)\left(n_{Z}^{2}+n_{N}^{2}\right)\right] \\
& +\frac{1}{4}\left[t_{1}\left(1+\frac{1}{2} x_{1}\right)+t_{2}\left(1+\frac{1}{2} x_{2}\right)\right] n\left(k^{2}+\frac{3}{5} k_{F}^{2}\right) \\
& -t_{0}\left(\frac{1}{2}+x_{0}\right) n_{\tau}-\frac{1}{6} t_{3} n^{\gamma}\left(\frac{1}{2}+x_{3}\right) n_{\tau} \\
& -\frac{1}{4}\left[t_{1}\left(\frac{1}{2}+x_{1}\right)-t_{2}\left(\frac{1}{2}+x_{2}\right)\right] n_{\tau}\left[k^{2}+\frac{3}{5} k_{F}^{2}(\tau)\right] .
\end{aligned}
$$

The $\mathrm{p}$ - $\mathrm{h}$ interaction is obtained by functional differentiation of the single particle potential energy or equivalently the double functional differentiation of the total potential energy, namely 


$$
\left\langle k_{1} k_{3}^{-1}\left|V_{p h}\right| k_{4} k_{2}^{-1}\right\rangle=\frac{\delta^{2}\langle V\rangle}{\delta n_{k_{3} k_{1}} \delta n_{k_{4} k_{2}}} .
$$

Hereafter, we will employ the standard notation for the participating momenta, namely $\mathbf{k}_{1}=\mathbf{q}+\mathbf{q}_{1}, \mathbf{k}_{2}=\mathbf{q}_{2}, \mathbf{k}_{3}=\mathbf{q}_{1}, \mathbf{k}_{4}=\mathbf{q}+\mathbf{q}_{2}$, which indicates that $\mathbf{q}$ is the transferred momentum. The $\mathrm{p}-\mathrm{h}$ interaction can be expressed as

$$
V_{p h}\left(\mathbf{q}_{1}, \mathbf{q}_{2}, \mathbf{q}\right)=\sum_{\left(\tau \tau^{\prime}, S\right)} V^{\left(\tau \tau^{\prime}, S\right)}\left(\mathbf{q}_{1}, \mathbf{q}_{2}, \mathbf{q}\right) P^{(S)}
$$

with the p-h spin projectors $P^{(0)}=1 / 2$ and $P^{(1)}=\vec{\sigma} \cdot \vec{\sigma}^{\prime} / 2$. The symbol $\left(\tau \tau^{\prime}\right)$ indicates the four isospin combinations

$$
\begin{aligned}
\left(p p^{-1} ; p p^{-1}\right) & \text { and } \quad\left(n n^{-1} ; n n^{-1}\right) \text { with } \tau^{\prime}=\tau, \\
\left(p p^{-1} ; n n^{-1}\right) & \text { and }\left(n n^{-1} ; p p^{-1}\right) \text { with } \tau^{\prime}=-\tau
\end{aligned}
$$

Two other isospin combinations, namely $\left(p n^{-1} ; p n^{-1}\right)$ and $\left(n p^{-1} ; n p^{-1}\right)$, are needed to describe the $\mathrm{p}-\mathrm{h}$ interaction relevant for the charge exchange processes. These are, however, not independent, and may be related to interactions in the $\left(p p^{-1} ; p p^{-1}\right),\left(n n^{-1} ; n n^{-1}\right)$, and $\left(p p^{-1} ; n n^{-1}\right)$ by isospin considerations. Denoting the $\mathrm{p}-\mathrm{h}$ interaction in the spin independent channels as $f_{p p}, f_{n n}$, and $f_{n p}$ for the particle hole states $\left(p p^{-1} ; p p^{-1}\right),\left(n n^{-1} ; n n^{-1}\right)$, and $\left(p p^{-1} ; n n^{-1}\right)$, respectively, we arrive at the relations [51]

$$
\begin{aligned}
f_{n n}= & t_{0}\left(1-x_{0}\right)+\frac{1}{6} t_{3} n^{\gamma}\left(1-x_{3}\right)+\frac{2}{3} \gamma t_{3} n^{\gamma-1}\left[\left(1+\frac{1}{2} x_{3}\right) n-\left(\frac{1}{2}+x_{3}\right) n_{n}\right] \\
& +\frac{1}{6} \gamma(\gamma-1) t_{3} n^{\gamma-2}\left[\left(1+\frac{1}{2} x_{3}\right) n^{2}-\left(\frac{1}{2}+x_{3}\right)\left(n_{p}^{2}+n_{n}^{2}\right)\right] \\
& +\frac{1}{4}\left[t_{1}\left(1-x_{1}\right)-3 t_{2}\left(1+x_{2}\right)\right] q^{2}+\frac{1}{4}\left[t_{1}\left(1-x_{1}\right)+3 t_{2}\left(1+x_{2}\right)\right] k_{F}(n)^{2}, \\
f_{n p}= & \frac{1}{2} t_{0}\left(x_{0}-1\right)+\frac{1}{12} t_{3} n^{\gamma}\left(x_{3}-1\right)+\frac{1}{8}\left[t_{1}\left(x_{1}-1\right)-t_{2}\left(1+x_{2}\right)\right] q^{2} \\
- & \frac{1}{8}\left[t_{1}\left(x_{1}-1\right)+t_{2}\left(1+x_{2}\right)\right]\left(k_{F}(n)^{2}+k_{F}(p)^{2}\right) .
\end{aligned}
$$

The interaction between $\mathrm{p}-\mathrm{h}$ proton states $f_{p p}$ is related to $f_{n n}$ evaluated at proton fraction $(1-x)$ by isospin symmetry. The spin dependent $\mathrm{p}$-h interactions characterized by $g_{n n}, g_{p p}$, and $g_{n p}$ are obtained by taking functional derivatives of the energy density with arbitrary spin excess and are given by

$$
\begin{aligned}
g_{n n} & =t_{0}\left(x_{0}-1\right)+\frac{1}{6} t_{3} n^{\gamma}\left(x_{3}-1\right)+\frac{1}{4}\left[t_{1}\left(x_{1}-1\right)-t_{2}\left(1+x_{2}\right)\right] q^{2} \\
& +\frac{1}{4}\left[t_{1}\left(x_{1}-1\right)+t_{2}\left(1+x_{2}\right)\right] k_{F_{n}}^{2}, \\
g_{n p} & =\frac{1}{2} t_{0} x_{0}+\frac{1}{12} t_{3} n^{\gamma} x_{3}+\frac{1}{8}\left[t_{1} x_{1}-t_{2} x_{2}\right] q^{2} \\
& +\frac{1}{8}\left[t_{1} x_{1}+t_{2} x_{2}\right]\left(k_{F}(n)^{2}+k_{F}(p)^{2}\right) .
\end{aligned}
$$

As noted earlier, the quantity $g_{p p}$ is equal to $g_{n n}$ evaluated at proton fraction $1-x$. 
In the schematic models of Ref. [41], one begins with a parametric form for the energy density for spin symmetric, but arbitrary isospin asymmetric, matter. The single particle potential, obtained by functional differentiation of the potential energy density, is given by

$$
\begin{aligned}
U_{i}(n, x, k ; T) & =\frac{1}{5} u\left[\sum_{i=1,2}\left\{5 C_{i} \pm\left(C_{i}-8 Z_{i}\right)(1-2 x)\right\}\right] g\left(k, \Lambda_{i}\right) \\
& +A u\left[1 \mp \frac{2}{3}\left(\frac{1}{2}+x_{0}\right)(1-2 x)\right] \\
& +B u^{\sigma}\left[1 \mp \frac{4}{3} \frac{1}{\sigma+1}(1-2 x)-\frac{2}{3} \frac{(\sigma-1)}{(\sigma+1)}\left(\frac{1}{2}+x_{3}\right)(1-2 x)^{2}\right] \\
& +\frac{2}{5} \frac{1}{n_{0}} \sum_{i=1,2}\left\{\left(2 C_{i}+4 Z_{i}\right) 2 \int \frac{d^{3} k}{(2 \pi)^{3}} g\left(k, \Lambda_{i}\right) f_{i}(k)\right. \\
& \left.+\left(3 C_{i}-4 Z_{i}\right) 2 \int \frac{d^{3} k}{(2 \pi)^{3}} g\left(k, \Lambda_{i}\right) f_{j}(k)\right\},
\end{aligned}
$$

where the upper (lower) sign in $\mp$ is for neutrons (protons) and $i \neq j$. Taking functional derivatives of the single particle potential energy density, we arrive at the p-h interaction parameters:

$$
f_{n n}=\frac{\delta U_{n}}{\delta n_{n}}, \quad f_{p p}=\frac{\delta U_{p}}{\delta n_{p}}, \quad f_{n p}=\frac{\delta U_{n}}{\delta n_{p}}=\frac{\delta U_{n}}{\delta n_{n}} .
$$

Their explicit algebraic forms are:

$$
\begin{aligned}
f_{n n} & =A\left(1-\frac{2}{3}\left(x_{0}+\frac{1}{2}\right)\right)+C \\
& +\left[1-\frac{4}{3} \frac{1}{\sigma+1}(\sigma(1-2 x)-2 x)\right] B u^{\sigma-1} \\
& -\left[\frac{2}{3} \frac{\sigma-1}{\sigma+1}\left(x_{3}+\frac{1}{2}\right)\left(\sigma(1-2 x)^{2}-4 x(1-2 x)\right)\right] B u^{\sigma-1} \\
& +\frac{2}{5}[(3 C-4 Z) x+(2 C+4 Z)(1-x)] \\
& -\frac{2}{3} \alpha\left[(3 \bar{C}-4 \bar{Z}) x^{2 / 3}+(2 \bar{C}+4 \bar{Z})(1-x)^{2 / 3}\right] u^{2 / 3} \\
& +\frac{2}{5} \alpha\left[(3 \bar{C}-4 \bar{Z}) x^{5 / 3}-(2 \bar{C}+4 \bar{Z})(1-x)^{5 / 3}\right] u^{2 / 3}+\frac{2}{5}(\tilde{C}-8 \tilde{Z}) x k_{F_{n}}{ }^{2}, \\
f_{n p} & =A\left(1+\frac{2}{3}\left(x_{0}+\frac{1}{2}\right)\right)+C \\
& +\left[1-\frac{4}{3} \frac{1}{\sigma+1}(\sigma(1-2 x)-2(1-x))\right] B u^{\sigma-1} \\
& -\left[\frac{2}{3} \frac{\sigma-1}{\sigma+1}\left(x_{3}+\frac{1}{2}\right)\left(\sigma(1-2 x)^{2}-4(1-x)(1-2 x)\right)\right] B u^{\sigma-1} \\
& +\frac{2}{5}[(3 C-4 Z) x+(2 C+4 Z)(1-x)] \\
& -\frac{2}{3} \alpha\left[(3 \bar{C}-4 \bar{Z}) x^{2 / 3}+(2 \bar{C}+4 \bar{Z})(1-x)^{2 / 3}\right] u^{2 / 3}
\end{aligned}
$$




$$
+\frac{2}{5} \alpha\left[(3 \bar{C}-4 \bar{Z}) x^{5 / 3}-(2 \bar{C}+4 \bar{Z})(1-x)^{5 / 3}\right] u^{2 / 3}+\frac{2}{5}(\tilde{C}-8 \tilde{Z}) k_{F_{n}}{ }^{2},
$$

with

$$
C=C_{1}+C_{2}, \quad \tilde{C}=\left(\frac{C_{1}}{\Lambda_{1}}+\frac{C_{2}}{\Lambda_{2}}\right), \quad Z=Z_{1}+Z_{2}, \quad \tilde{Z}=\left(\frac{Z_{1}}{\Lambda_{1}}+\frac{Z_{2}}{\Lambda_{2}}\right) .
$$

The form of the energy density employed in these schematic models does not explicitly account for the explicit spin dependence of the nucleon-nucleon interaction. The potential energy density is independent of any spin excess indicating that $g_{n n}$ and $g_{n p}$ are zero.

The p-h interactions parameters discussed in this appendix are related to the Fermi-liquid parameters. In symmetric nuclear matter the appropriate relations are

$$
f_{n n}=\frac{F_{0}+F_{0}^{\prime}}{N_{0}}, \quad f_{n p}=\frac{F_{0}-F_{0}^{\prime}}{N_{0}}, \quad g_{n n}=\frac{G_{0}+G_{0}^{\prime}}{N_{0}}, \quad g_{n p}=\frac{G_{0}-G_{0}^{\prime}}{N_{0}},
$$

where the normalization factor $N_{0}=2 M^{*} k_{F} / \pi^{2}$ is the density of states at the Fermi surface. For neutron matter $F_{0}=N_{0} f_{n n}$ and $G_{0}=g_{n n} N_{0}$, with $N_{0}=M^{*} k_{F} / \pi^{2}$.

\section{APPENDIX B: POLARIZATION FUNCTIONS}

The various polarization functions required to evaluate the Hartree and RPA response functions are presented in this appendix. The zero temperature polarization functions may be found in Ref. 34] and for finite temperatures in Ref. [35]. Here, we collect the present extensions of these results to asymmetric matter, and, in particular, to unlike p-h excitations. For space like excitations, $q_{\mu}^{2} \leq 0$, they are given by

$$
\begin{aligned}
\operatorname{Im} \Pi_{L}\left(q_{0}, \vec{q}\right) & =2 \pi \int \frac{d^{3} p}{(2 \pi)^{3}} \frac{E_{p}^{* 2}-|p|^{2} \cos ^{2} \theta}{E_{p}^{*} E_{p+q}^{*}} \Theta, \\
\operatorname{Im} \Pi_{T}\left(q_{0}, \vec{q}\right) & =\pi \int \frac{d^{3} p}{(2 \pi)^{3}} \frac{q_{\mu}^{2} / 2-|p|^{2}\left(1-\cos ^{2} \theta\right)}{E_{p}^{*} E_{p+q}^{*}} \Theta, \\
\operatorname{Im} \Pi_{A}\left(q_{0}, \vec{q}\right) & =2 \pi \int \frac{d^{3} p}{(2 \pi)^{3}} \frac{M_{2}^{*^{2}}}{E_{p}^{*} E_{p+q}^{*}} \Theta, \\
\operatorname{Im} \Pi_{V A}\left(q_{0}, \vec{q}\right) & =2 \pi \int \frac{d^{3} p}{(2 \pi)^{3}} \frac{q_{\mu}^{2} M_{2}^{*}}{\left|q^{2}\right| E_{p}^{*} E_{p+q}^{*}} \Theta .
\end{aligned}
$$

In the above,

$$
\begin{aligned}
& \Theta=F\left(E_{p}^{*}, E_{p+q}^{*}\right)\left[\delta\left(q_{0}-\left(E_{p+q}-E_{p}\right)\right)+\delta\left(q_{0}-\left(E_{p}-E_{p+q}\right)\right],\right. \\
& F\left(E_{p}^{*}, E_{p+q}^{*}\right)=f_{2}\left(E_{p}^{*}\right)\left(1-f_{4}\left(E_{p+q}^{*}\right)\right), \\
& E_{p}^{*}=\sqrt{|p|^{2}+M_{2}^{* 2}}, \quad E_{p}=E_{p}^{*}+U .
\end{aligned}
$$

The particle distribution functions $f_{i}(E)$ are given by the Fermi-Dirac distribution functions

$$
f_{i}\left(E_{p}^{*}\right)=\left(1+\exp \left[\left(E_{p}^{*}-\nu_{i}\right) / k T\right]\right)^{-1},
$$


where $\nu$ is the effective chemical potential defined by

$$
\nu_{i}=\mu_{i}-U_{i}=\mu_{i}-\left(g_{\omega B_{i}} \omega_{0}+t_{3 B_{i}} g_{\rho B_{i}} b_{0}\right),
$$

and the particle labels 2 and 4 correspond to the initial and final baryons.

The angular integrals are performed by exploiting the delta functions. The three dimensional integrals can be reduced to the following one dimensional integrals:

$$
\begin{aligned}
\operatorname{Im} \Pi_{L}\left(q_{0}, q\right) & =\frac{q_{\mu}^{2}}{2 \pi|q|^{3}} \int_{e_{-}}^{\infty} d E\left[\left(E+q_{0} / 2\right)^{2}-|q|^{2} / 4\right] \\
& \times\left[F\left(E, E+q_{0}\right)+F\left(E+q_{0}, E\right)\right] \\
\operatorname{Im} \Pi_{T}\left(q_{0}, q\right) & \left.=\frac{q_{\mu}^{2}}{4 \pi|q|^{3}} \int_{e_{-}}^{\infty} d E\left[\left(E^{*}+q_{0} / 2\right)^{2}+|q|^{2} / 4+|q|^{2} M_{2}^{*^{2}} / q_{\mu}^{2}\right)\right] \\
& \times\left[F\left(E, E+q_{0}\right)+F\left(E+q_{0}, E\right)\right], \\
\operatorname{Im} \Pi_{A}\left(q_{0}, q\right) & =\frac{M_{2}^{*^{2}}}{2 \pi|q|} \int_{e_{-}}^{\infty} d E\left[F\left(E, E+q_{0}\right)+F\left(E+q_{0}, E\right)\right], \\
\operatorname{Im} \Pi_{V A}\left(q_{0}, q\right) & =\frac{q_{\mu}^{2}}{8 \pi|q|^{3}} \int_{e_{-}}^{\infty} d E\left[2 E+q_{0}\right] \\
& \times\left[F\left(E, E+q_{0}\right)+F\left(E+q_{0}, E\right)\right] .
\end{aligned}
$$

The lower cut-off $e_{-}$arises due to kinematical restrictions and is given by

$$
e_{-}=-\beta \frac{\tilde{q_{0}}}{2}+\frac{q}{2} \sqrt{\beta^{2}-4 \frac{M_{2}^{* 2}}{q^{2}-\tilde{q}_{0}^{2}}}
$$

where

$$
\tilde{q_{0}}=q_{0}+U_{2}-U_{4}, \quad \beta=1+\frac{M_{4}^{*^{2}}-M_{2}^{*^{2}}}{q^{2}-\tilde{q}_{0}^{2}}
$$

It is convenient to re-express the polarization functions as follows:

$$
\begin{aligned}
\operatorname{Im} \Pi_{L}^{R}\left(q_{0}, q\right) & =\frac{q_{\mu}^{2}}{2 \pi|q|^{3}}\left[I_{2}+q_{0} I_{1}+\frac{q_{\mu}^{2}}{4} I_{0}\right] \\
\operatorname{Im} \Pi_{T}^{R}\left(q_{0}, q\right) & =\frac{q_{\mu}^{2}}{4 \pi|q|^{3}}\left[I_{2}+q_{0} I_{1}+\left(\frac{q_{\mu}^{2}}{4}+\frac{q^{2}}{2}+M_{2}^{*^{2}} \frac{q^{2}}{q_{\mu}^{2}}\right) I_{0}\right] \\
\operatorname{Im} \Pi_{A}^{R}\left(q_{0}, q\right) & =\frac{M_{2}^{*^{2}}}{2 \pi|q|} I_{0} \\
\operatorname{Im} \Pi_{V A}^{R}\left(q_{0}, q\right) & =\frac{q_{\mu}^{2}}{8 \pi|q|^{3}}\left[q_{0} I_{0}+2 I_{1}\right]
\end{aligned}
$$

where we used the one-dimensional integrals

$$
I_{n}=\tanh \left(\frac{q_{0}+\left(\mu_{2}-\mu_{4}\right)}{2 T}\right) \int_{e_{-}}^{\infty} d E E^{n}\left[F\left(E, E+q_{0}\right)+F\left(E+q_{0}, E\right)\right]
$$


These integrals may be explicitly expressed in terms of the Polylogarithmic functions

$$
L i_{n}(z)=\int_{0}^{z} \frac{L i_{n-1}(x)}{x} d x, \quad L i_{1}(x)=\ln (1-x)
$$

which are defined to conform to the definitions of Lewin [50]. This Polylogarithm representation is particularly useful and compact:

$$
\begin{aligned}
I_{0} & =T z\left(1+\frac{\xi_{1}}{z}\right), \\
I_{1} & =T^{2} z\left(\frac{\mu_{2}-U_{2}}{T}-\frac{z}{2}+\frac{\xi_{2}}{z}+\frac{e_{-} \xi_{1}}{z T}\right), \\
I_{2} & =T^{3} z\left(\frac{\left(\mu_{2}-U_{2}\right)^{2}}{T^{2}}-z \frac{\mu_{2}-U_{2}}{T}+\frac{\pi^{2}}{3}+\frac{z^{2}}{3}\right) \\
& -T^{3} z\left(2 \frac{\xi_{3}}{z}-2 \frac{e_{-} \xi_{2}}{T z}-\frac{e_{-}^{2} \xi_{1}}{T^{2} z}\right),
\end{aligned}
$$

where $z=\left(q_{0}+\left(\mu_{2}-\mu_{4}\right)\right) / T$ and the factors $\xi_{n}$ are given by

$$
\xi_{n}=L i_{n}\left(-\alpha_{1}\right)-L i_{n}\left(-\alpha_{2}\right),
$$

with

$$
\alpha_{1}=\exp \left(\left(e_{-}-\mu_{2}+U_{2}\right) / T\right), \quad \alpha_{2}=\exp \left(\left(e_{-}+q_{0}-\mu_{4}+U_{2}\right) / T\right) .
$$

We note that the nonrelativistic structure function for neutral current scattering, Eq. (3) is, aside from the factor $M_{2}^{*^{2}} T / \pi q$, equal to $I_{0}$ since $\xi_{1} \equiv \xi_{-}$. In the case of neutral currents, some of the terms in the above are simplify,

$$
z=\frac{q_{0}}{T}, \quad \mu_{2}=\mu_{4}, \quad e_{-}=-\frac{q_{0}}{2}+\frac{q}{2} \sqrt{1-4 \frac{M_{2}^{* 2}}{q_{\mu}^{2}}},
$$

and we recover the results obtained earlier in Ref. [35].

For the real part, analytic forms do not exist. In the following we present, in integral form, the required polarization functions. The results presented below generalize the results of Ref. [35] to the case when the particle and hole states correspond to different baryons.

$$
\begin{aligned}
& \operatorname{Re} \Pi_{L}=-\frac{4}{\pi^{3}} \int d^{3} p \frac{\mathrm{F}\left(E_{p}^{*}\right)}{2 E_{p}^{*}}\left[\frac{q_{\mu}^{2}\left(E_{p}^{* 2}-|\vec{p}|^{2} \cos ^{2} \theta\right)}{q_{\mu}^{4}-4(p \cdot q)^{2}}\right]_{p_{0}=E_{p}^{*}} \\
&= \frac{-q_{\mu}^{2}}{\pi^{2}|\vec{q}|^{3}} \int_{0}^{\infty} d p \frac{p}{E_{p}^{*}} \mathrm{~F}\left(E_{p}^{*}\right) \\
& \quad \times \quad\left[|\vec{p}||\vec{q}|-\frac{1}{2}\left(\left(E_{p}^{* 2}-q_{0} E_{p}^{*}+q_{\mu}^{2} / 4\right) L_{-}-\left(E_{p}^{* 2}+q_{0} E_{p}^{*}+q_{\mu}^{2} / 4\right) L_{+}\right)\right], \\
& \operatorname{Re} \Pi_{T}=-\frac{4}{\pi^{3}} \int d^{3} p \frac{\mathrm{F}\left(E_{p}^{*}\right)}{2 E_{p}^{*}}\left[\frac{\left.(p \cdot q)^{2}-q_{\mu}^{2} *|\vec{p}|^{2}\left(1-\cos ^{2} \theta\right) / 2\right)}{q_{\mu}^{4}-4(p \cdot q)^{2}}\right]_{p_{0}=E_{p}^{*}}
\end{aligned}
$$




$$
\begin{aligned}
&= \frac{1}{\pi^{2}|\vec{q}|^{3}} \int_{0}^{\infty} d p \frac{p}{E_{p}^{*}} \mathrm{~F}\left(E_{p}^{*}\right)\left[|\vec{q}|^{3}|\vec{p}|+|\vec{q}||\vec{p}| q_{\mu}^{2} / 2\right. \\
&-\left.\frac{q_{\mu}^{2}}{4}\left(\left(E_{-}^{2}+M^{* 2} \frac{|\vec{q}|^{2}}{q_{\mu}^{2}}+\frac{|\vec{q}|^{2}}{4}\right) L_{-}+\left(E_{+}^{2}+M^{* 2} \frac{|\vec{q}|^{2}}{q_{\mu}^{2}}+\frac{|\vec{q}|^{2}}{4}\right) L_{+}\right)\right], \\
& \operatorname{Re} \Pi_{S}=-\frac{4}{\pi^{3}} \int d^{3} p \frac{\mathrm{F}\left(E_{p}^{*}\right)}{2 E_{p}^{*}}\left[\frac{q_{\mu}^{2} M^{* 2}-(p \cdot q)^{2}}{q_{\mu}^{4}-4(p \cdot q)^{2}}\right]_{p_{0}=E_{p}^{*}} \\
&=-\frac{1}{\pi^{2}|\vec{q}|} \int_{0}^{\infty} d p \frac{p}{E_{p}^{*}} \mathrm{~F}\left(E_{p}^{*}\right)\left[|\vec{q}||\vec{p}|-\left(q_{\mu}^{2}-4 M^{* 2}\right)\left(L_{-}+L_{+}\right) / 8\right], \\
& \quad \operatorname{Re} \Pi_{A}=-\frac{4}{\pi^{3}} \int^{3} p \frac{\mathrm{F}\left(E_{p}^{*}\right)}{2 E_{p}^{*}}\left[\frac{q_{\mu}^{2} M^{* 2}}{q_{\mu}^{4}-4(p \cdot q)^{2}}\right]_{p_{0}=E_{p}^{*}} \\
& \operatorname{Re} \Pi_{M}=-\frac{4 M^{2}|\vec{q}|}{\pi^{3}} \int_{0}^{\infty} d p \frac{p}{E_{p}^{*}} \mathrm{~F}_{+}\left(E_{p}^{*} p \frac{\mathrm{F}\left(E_{p}^{*}\right)}{2 E_{p}^{*}}\left[\frac{q_{\mu}^{2} E_{p}^{*}-L_{-}(p \cdot q)}{q_{\mu}^{4}-4(p \cdot q)^{2}}\right]_{p_{0}=E_{p}^{*}},\right. \\
&=-\frac{M^{*}}{2 \pi^{2}|\vec{q}|} \int_{0}^{\infty} d p \frac{p}{E_{p}^{*}} \mathrm{~F}\left(E_{p}^{*}\right)\left[E_{-} L_{-}+E_{+} L_{+}\right],
\end{aligned}
$$

where

$$
\begin{aligned}
\mathrm{F}(E) & =\frac{f_{i}(E)+f_{j}(E)}{2}, \quad E_{ \pm}=E_{p}^{*} \pm q_{0} / 2 \\
L_{+} & =\ln \left[1+\frac{4|\vec{q}||\vec{p}|}{2 E_{p}^{*} q_{0}-q_{\mu}^{2}+2|\vec{q}||\vec{p}|}\right] \frac{f_{i}\left(E_{p}^{*}\right)}{\mathrm{F}(E)} \\
L_{-} & =\ln \left[1-\frac{4|\vec{q}||\vec{p}|}{2 E_{p}^{*} q_{0}+q_{\mu}^{2}-2|\vec{q}||\vec{p}|}\right] \frac{f_{j}\left(E_{p}^{*}\right)}{\mathrm{F}(E)} .
\end{aligned}
$$

The particle labels $i$ and $j$ refer to the initial and final state baryon. Anti-particle contributions have been neglected in the above formulae as they provide negligible contributions for the temperatures and densities of interest. Since the typical temperatures never exceed a few tens of MeV's they will be only important for electrons and only when $\mu_{e} \ll T$.

The vacuum contributions, which only play a role in the relativistic Hartree approximation, are not explicitly given here, but may be found in Ref. [8]. 


\section{REFERENCES}

[1] S. Reddy, M. Prakash, and J. M. Lattimer, Phys. Rev. D58, (1998) 013009.

[2] R. F. Sawyer, Phys. Rev. D11, (1975) 2740; Phys. Rev. C40, (1989) 865.

[3] N. Iwamoto and C. J. Pethick, Phys. Rev. D25, (1982) 313.

[4] D. Pines and D. Bohm, Phys. Rev. 92, (1953) 608.

[5] L.D. Landau, Zh. Eksp. Teor. Fiz. 30, (1956) 1058. [Sov. Phys. JETP 3 (1957) 920]; Zh. Eksp. Teor. Fiz. 32, (1957) 59; [Sov. Phys. JETP 5, (1957) 102.

[6] P. Noziéres, Theory of Interacting Fermi Systems, (Benjamin, New York, 1964).

[7] G. Baym and C. Pethick, Landau Fermi-liquid Theory (John Wiley \& Sons, New York, 1991).

[8] C. J. Horowitz and K. Wehrberger, Nucl. Phys. A531, (1991) 665; Phys. Rev. Lett. 66, (1991) 272; Phys. Lett. B226, (1992) 236.

[9] G. Fabbri and F. Matera, Phys. Rev. C54, (1996) 2031.

[10] S. Reddy, J. Pons, M. Prakash, and J.M. Lattimer, in Stellar Evolution, Stellar Explosions, and Galactic Chemical Evolution, ed. A. Mezzacappa, (IOP Publishing: Bristol, 1998) p. 585.

[11] M. Prakash and S. Reddy, in Nuclear Astrophysics, eds. M. Bublla, W. Nörenberg, J. Wambach, and A. Wirzba, (GSI: Darmstadt, 1998) p. 201.

[12] A. Burrows and R.F Sawyer, Phys. Rev. C C58, (1998) 554; Phys. Rev. C (1998) in press astro-ph/9804264).

[13] S. Hannestad and G. Raffelt, Astrophys. Jl., (1998), 507, 339.

[14] D. H. Wilkinson, Phys. Rev. C7, (1973) 930; M. Rho, Nucl. Phys. A231, (1974) 493.

[15] G. E. Brown and M. Rho, Phys. Rev. Lett. 66, (1991) 2720.

[16] G. Bertsch and S.F. Tsai, Phys. Rep. 18, (1975) 125.

[17] J. Lindhard, Kgl. Danske Videnskab. Selskab, Mat-fys. Medd, 28 (1954) 8.

[18] A.L. Fetter and J.D. Walecka, Quantum Theory of Many Particle Systems (New York: McGraw-Hill, 1971).

[19] S. Doniach and E. H. Sondheimer, Green's Functions for Solid State Physicists (Reading, The Benjamin/Cummings Publishing Company, Inc. 1974).

[20] F. C. Khanna and H. R. Glyde, Can. Jl. Phys. 54 (1976) 648.

[21] S.-O. Bäckmann, G.E. Brown, and J.A. Niskanen Phys. Rep. 124, (1985) 1.

[22] G. E, Brown, Unified Theory of Nuclear Models and Forces (North-Holland, Amsterdam, 1971).

[23] D. Vautherin and D.M. Brink, Phys. Rev. C5, (1972) 626.

[24] V. G. Nguyen and H. Sagawa, Phys. Lett. B 106, (1981) 379.

[25] E. Chabanat, P. Bonche, P. Haensel, J. Meyer, and R. Schaeffer, Nucl. Phys. A 627, (1997) 710.

[26] M. Kutschera, and W. Wojcik, Phys. Lett. B 325, (1994) 271.

A. Viduarre, J. Navarro, and J. Bernabeu, Astron. Astrophys. 135, (1984) 361.

[27] R. B. Wiringa, V. Fiks, and A. Fabrocine, Phys. Rev. C38, (1988) 1010.

[28] A. D. Jackson, E. Krotscheck, D.E. Meltzer, and R. A. Smith Nucl. Phys. A 386, (1992) 125.

[29] S.O. Bäckmann and C.-G. Källman, Phys. Lett. B43, (1973) 263. 
[30] A. Akmal and V.R. Pandharipande, Phys. Rev. C C56, (1997) 2261.

[31] A. Akmal, V. R. Pandharipande, and D.G. Ravenhall, Phys. Rev. C 58, (1998) 1804

[32] B. D. Serot and J. D. Walecka, Advances in Nuclear Physics, 16, eds. J. W. Negele and E. Vogt, (New York: Plenum); B.D. Serot, Rep. Prog. Phys. 55, (1992) 1855.

[33] K. Wehreberger Phys. Rep. 225, (1993) 273.

[34] K. Lim and C. J. Horowitz, Nucl. Phys. A501, (1989) 729.

[35] K. Saito, T. Maruyama, and K. Soutame, Phys. Rev. C 40, (1989) 407.

[36] H. Kim, J. Piekarewicz, and C.J. Horowitz, Phys. Rev. C 51, (1995) 2739.

[37] A.B. Migdal, Theory of Finite Fermi Systems and Applications to Atomic Nuclei (New York: Interscience, 1962).

[38] J.P. Blaizot, Phys. Rep. 64, (1980) 171.

[39] I.S. Towner Phys. Rep. 155, (1987) 263.

[40] W.G. Love snd J. Speth, Comments Nucl. Part. Phys. 14, (1985) 185.

[41] M. Prakash, I. Bombaci, Manju Prakash, P. J. Ellis, J. M. Lattimer, and R. Knorren, Phys. Rep 280, (1997) 1.

[42] H.C. Chiang, E. Oset, R.C. Carrasco, J. Nieves, and J. Navarro Nucl. Phys. A510, (1990) 573 .

[43] A.B. Migdal, E.E. Sapersrein, M.A. Troitsky, and D.N. Voskresensky Phys. Rep. 192, (1990) 179.

[44] E. Oset, H. Toki, and W. Weise, Phys. Rep. 83, (1982) 280.

[45] S. A. Chin, Annals of Physics 108, (1989) 407.

[46] N. K. Glendenning and S. Moszkowski, Phys. Rev. Lett. 67, (1991) 2414.

[47] A. Burrows and J. M. Lattimer, Astrophys. Jl., 307, (1986) 178.

[48] W. Keil and H. T. Janka, Astronomy and Astrophysics 296, (1995) 145.

[49] J. Pons, S. Reddy, M. Prakash, J. M. Lattimer, and J. A. Miralles, Astrophys. Jl., (1998) in press.

[50] L. Lewin, Polylogarithms and Associated Functions (New York: North-Holland, 1983).

[51] E.S. Hernández, J. Navarro, and A. Polls. Nucl. Phys. A627, (1996) 460 . 


\section{FIGURE CAPTIONS}

FIG. 1: The ratio of the neutrino mean free paths computed with $\left(M^{*}\right)$ and without $(M)$ effective mass corrections as a function of nucleon density $n_{B}$ in units of the nuclear equilibrium density $n_{0}=0.16 \mathrm{fm}^{-3}$. Results are shown for pure neutron matter at $T=5$ and $30 \mathrm{MeV}$. The dash-dot curve shows the density dependence of the neutron effective mass.

FIG. 2: The density dependence of the Fermi-liquid parameters and the neutron effective mass for neutron matter for Skyrme models studied in this work.

FIG. 3: The density dependence of Fermi-liquid parameters of microscopic calculations from Refs. 28] and 29] (see text for details).

FIG. 4: The neutrino differential cross sections in neutron matter as a function of the ratio of energy to momentum transfer for $T=0 \mathrm{MeV}$ (left panels) and $T=10 \mathrm{MeV}$ (right panels) at $n_{0}$ (top panels) and $n_{0} / 4$ (bottom panels). Results are for the Skyrme model SLy4 for $q=E_{\nu}=25 \mathrm{MeV}$. The symbols $V$ and $A$ refer to the vector and axial vector contributions, respectively. Thin lines refer to the Hartree results and thick lines to the $\mathrm{RPA}$ results.

FIG. 5: Same as Fig. 4, except that results are for the model of Backmann et al. [29] and are shown for three densities: $2 n_{0}$ (top panels), $n_{0}$ (center panels), and $n_{0} / 4$ (lower panels).

FIG. 6: The density dependence of the neutrino mean free paths at energy $E_{\nu}=\pi T$ in neutron matter for $T=10,20$ and $30 \mathrm{MeV}$. Results for the microscopic calculations of Bäckmann et al. are in the top panels and those for the Skyrme model SLy4 are in the bottom panels. The right panels show the extent to which RPA corrections modify the Hartree results.

FIG. 7: The density dependence of the neutrino mean free paths in the relativistic models RHA and GM3 in the Hartree approximation (i.e., RPA correlations were ignored) at energy $E_{\nu}=\pi T$ for $T=0,10$ and $20 \mathrm{MeV}$. For reference, the results for the case in which effective mass corrections are ignored are also shown as dashed lines.

FIG. 8: The neutrino differential cross sections in neutron matter in the model RHA for $q=E_{\nu}=25 \mathrm{MeV}$. Dotted lines show the Hartree results, while solid, dashed and dot-dashed curves show the results including RPA correlations with $g^{\prime}=0,0.3$ and 0.6 , respectively. The left panels have $T=0 \mathrm{MeV}$ and right panels have $T=10 \mathrm{MeV}$; the top panels are for $4 n_{0}$ and bottom panels are for $n_{0}$.

FIG. 9: Same as in Fig. 8, but for the model GM3.

FIG. 10: The density and temperature dependences of neutrino mean free paths at energy $E_{\nu}=\pi T$ in neutron matter. The top panels are for the model GM3; the bottom panels are for RHA. The left panels show the results in the Hartree approximation. In the right panels, effects due to spin dependent correlations introduced through the Migdal parameter $g^{\prime}$ are 
shown. The different curves correspond to the same temperatures as in the left panels.

FIG. 11: The neutrino differential cross sections in symmetric nuclear matter for $q=$ $E_{\nu}=30 \mathrm{MeV}$. The Fermi-liquid parameters employed are given in Eq. (36). Results for the free gas, the Hartree approximation, and with RPA correlations are compared for $T=0$ and $10 \mathrm{MeV}$.

FIG. 12: The temperature dependence of the neutrino scattering mean free path in symmetric nuclear matter at density $n_{0}$ for the Fermi-liquid parameters in Eq. (36). The left panel shows results for thermal neutrinos $\left(E_{\nu}=\pi T\right)$ calculated in the Hartree approximation, and the right panel shows the effect of RPA correlations.

Fig. 13: The density dependence of the effect of RPA correlations on the neutral current neutrino mean free paths in the nonrelativistic SLn2 models with different compressibilities.

FIG. 14: The density dependence of the neutrino scattering mean free path in symmetric nuclear matter for the EOS SL22 at $T=10,20$, and $30 \mathrm{MeV}$. The upper panel shows results for thermal neutrinos $\left(E_{\nu}=\pi T\right)$ calculated in the Hartree approximation, and the lower panel shows the effect of RPA correlations.

FIG. 15: The density dependence of the Fermi-liquid and effective nucleon mass in symmetric nuclear matter for Skyrme models used in this paper.

FIG. 16: The neutrino scattering mean free paths in symmetric nuclear matter for the Skyrme models SGII (top panels) and SLy4 (bottom panels), at a neutrino energy $E_{\nu}=$ $\pi T$. Left panels show the Hartree approximation and right panels show the effect of RPA correlations.

FIG. 17: Differential scattering cross sections in $\beta$-stable neutrino-free matter for the schematic model SL22. The proton fraction $x_{p}=0.049$ for $n_{B}=n_{0}$ (lower panels) and $x_{p}=0.22$ at $n_{B}=3 n_{0}$ (upper panels). The left panels show results for $T=0$ while right panels are for $T=10 \mathrm{MeV} . E_{\nu}=q=30 \mathrm{MeV}$ are assumed.

FIG. 18: Differential scattering cross sections in $\beta$-stable neutrino-free matter for the Skyrme models SLy4 (top panels) and SGII (bottom panels), for the indicated proton fractions and temperatures. Results for both the Hartree approximation (dashed lines) and with RPA correlations (solid lines) are displayed.

FIG. 19: The density dependence of the scattering mean free paths in $\beta$-stable asymmetric matter for the schematic model SL22 at the indicated temperatures, in the Hartree approximation (left panel) and with RPA correlations included (right panel). In the right panel, thick lines show results obtained by employing empirical values for the spin parameters. Thin lines correspond to results obtained without any spin-dependent $\mathrm{p}$-h interaction.

FIG. 20: Differential cross sections at $n_{B}=0.32 \mathrm{fm}^{-3}$ in $\beta$-stable neutrino-free matter for the field-theoretical models RHA (left panels) and GM3 (right panels) for $T=0$ (lower panels) and $T=10 \mathrm{MeV}$ (upper panels). Results for the Hartree approximation alone and also including RPA corrections with $g^{\prime}=0,0.1$ and 0.6 are compared. 
FIG. 21: The density and temperature dependences of the neutral current mean free paths for $\beta$-stable neutrino free matter in the field-theoretical model GM3. The upper left panel shows the Hartree results for the case $E_{\nu}=3 T$. The influence of the spin correlations introduced via the Migdal parameter $g^{\prime}$ is strong, as can be deduced from the results shown in the upper right and bottom panels.

FIG. 22: Charged current diferential cross sections in symmetric nuclear matter at nuclear saturation density, for the case $E_{\nu}=q=50 \mathrm{MeV}$. Empirical values of the Fermi-liquid parameters are employed, and results for the Hartree approximation and with RPA correlations are compared. For $T=0$ (left panel), the peaks labelled 'GD' and 'GT' correspond to the giant dipole and Gamow-Teller resonances, respectively. For $T=10 \mathrm{MeV}$ (right panel), the collective states are significantly broadened and overlap.

FIG. 23: Charged current differential cross sections in $\beta$-stable matter for $Y_{L}=0.4$ and $T=10 \mathrm{MeV}$ at $n_{B}=n_{0}$ (left panel) and $n_{B}=3 n_{0}$ (right panel). The schematic model SL22 was employed. Results for the Hartree approximation and with RPA correlations are compared for the case $q=E_{\nu}=\mu_{\nu}$.

FIG. 24: The density and temperature dependences of the charged current neutrino mean free path in $\beta$-stable matter for the SL22 model assuming $Y_{L}=0.4$. Results for the Hartree approximation (left panel) are compared with those including RPA corrections (right panel) with $g^{\prime}=0.6$.

FIG. 25: Same as Fig. 21, except for neutrino-free matter and for $q=E_{\nu}+\mu_{e}$ and $E_{\nu}=\pi T$.

FIG. 26: Same as Fig. 22, except for neutrino-free matter and for $q=E_{\nu}+\mu_{e}$ and $E_{\nu}=\pi T$.

FIG. 27: Charged current differential cross sections in $\beta$-stable matter for $Y_{L}=0.4$ and $T=10 \mathrm{MeV}$ at $n_{B}=n_{0}$ (left panel) and $n_{B}=3 n_{0}$ (right panel). The relativistic model GM3 was employed. Results for the Hartree approximation and with RPA correlations are compared for the case $q=E_{\nu}=\mu_{\nu}$.

FIG. 28: The density and temperature dependences of the charged current neutrino mean free path in $\beta$-stable matter for the GM3 model assuming $Y_{L}=0.4$. Results for the Hartree approximation (left panel) are compared with those including RPA corrections (right panel) with $g^{\prime}=0.6$.

FIG. 29: Same as Fig. 27, except for neutrino-free matter and for $q=E_{\nu}+\mu_{e}$ and $E_{\nu}=\pi T$.

FIG. 30: Same as Fig. 28, except for neutrino-free matter and for $q=E_{\nu}+\mu_{e}$ and $E_{\nu}=\pi T$.

FIG. 31: RPA corrections to the diffusion coefficients in the density-temperature plane for the field-theoretical model GM3. Results are shown for the three diffusion coefficients $D_{2}, D_{3}$, and $D_{4}$ (upper, middle, and lower panels, respectively) for neutrino-free matter (left 
panels) and neutrino-rich matter (right panels).

FIG: 32: The density and temerature dependence of the ratio of the RPA and Hartree diffusion coefficients $\left(D_{i}^{R P A} / D_{i}^{H}\right)$ in neutrino-free matter.

FIG: 33: Central values of thermodynamic quantities (entropy $s$, temperature $T$, baryon density $n_{B}, \nu_{e}$ concentration $Y_{\nu}, \nu_{e}$ chemical potential $\mu_{\nu}$, electron concentration $Y_{e}$ ) during the evolution of a baryon mass $1.4 \mathrm{M}_{\odot}$ protoneutron star. The relativistic model GM3 for nucleons-only matter was employed. Results are compared for the Hartree approximation (baseline case), the inclusion of RPA correlations, and a constant factor of three reduction of the opacities for densities greater than $u=n_{B} / n_{0}=1 / 2$ and $1 / 4$. The latter case corresponds to that studied in Ref. [12].

FIG 34: The upper panel shows the total emitted neutrino luminosity for the protoneutron star evolutions described in Fig. 33. The curves are labelled as in Fig. 32. The lower panel shows the ratio of the luminosities obtained in the three models which contain corrections to the baseline (Hartree approximation) model.

FIG 35: The total emitted neutrino luminosity for long-term protoneutron star evolutions described in Fig. 34. The curves are labelled as in Fig. 34. 


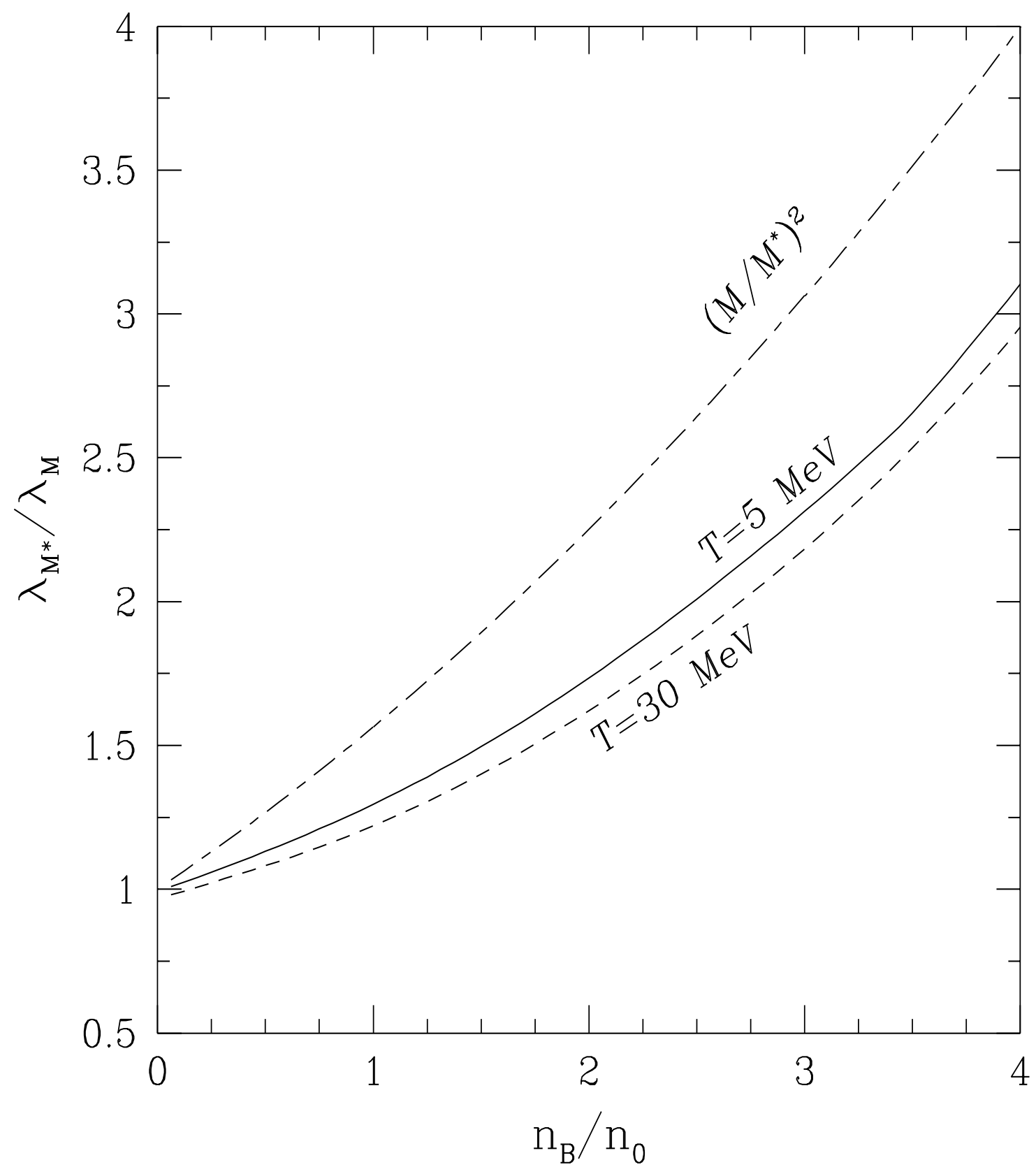

FIG. 1. 

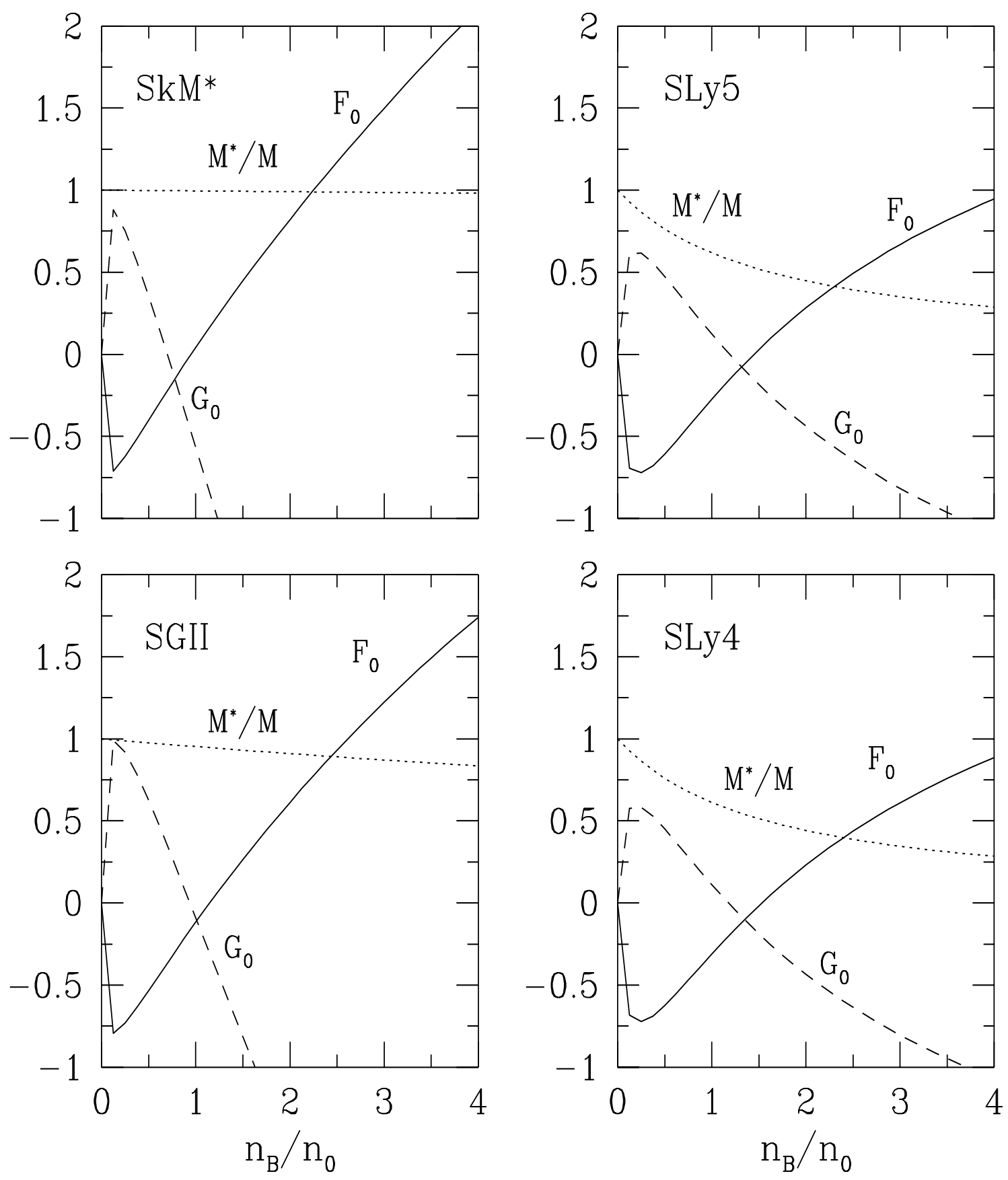

FIG. 2. 


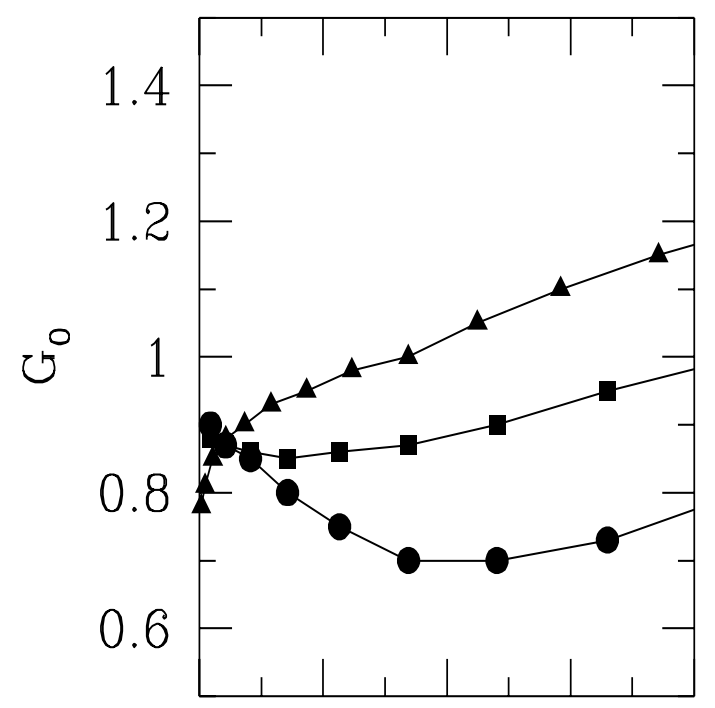

- Reid v6

- BJ $\mathrm{v} 6$

- Bàckmann et al.
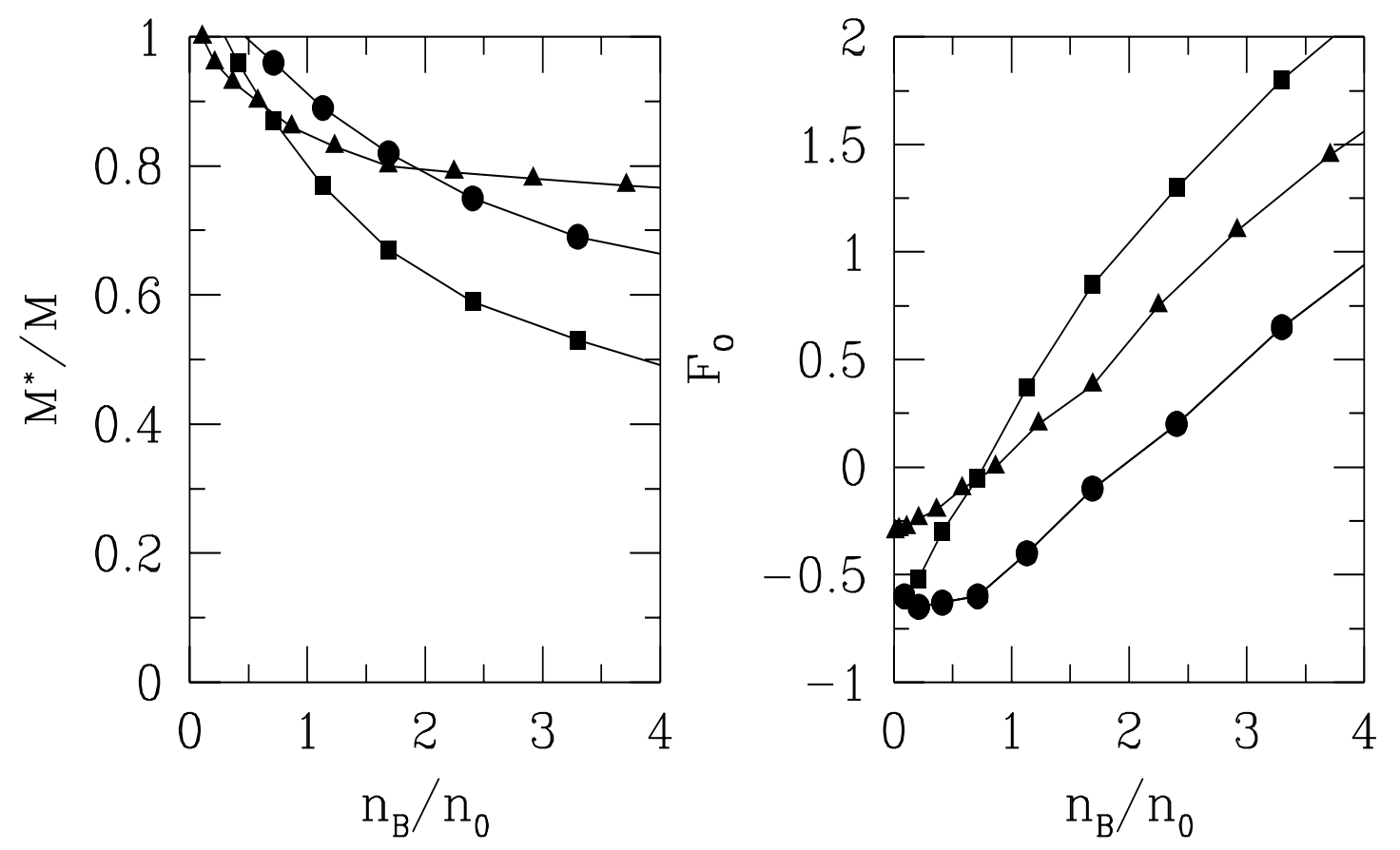

FIG. 3. 


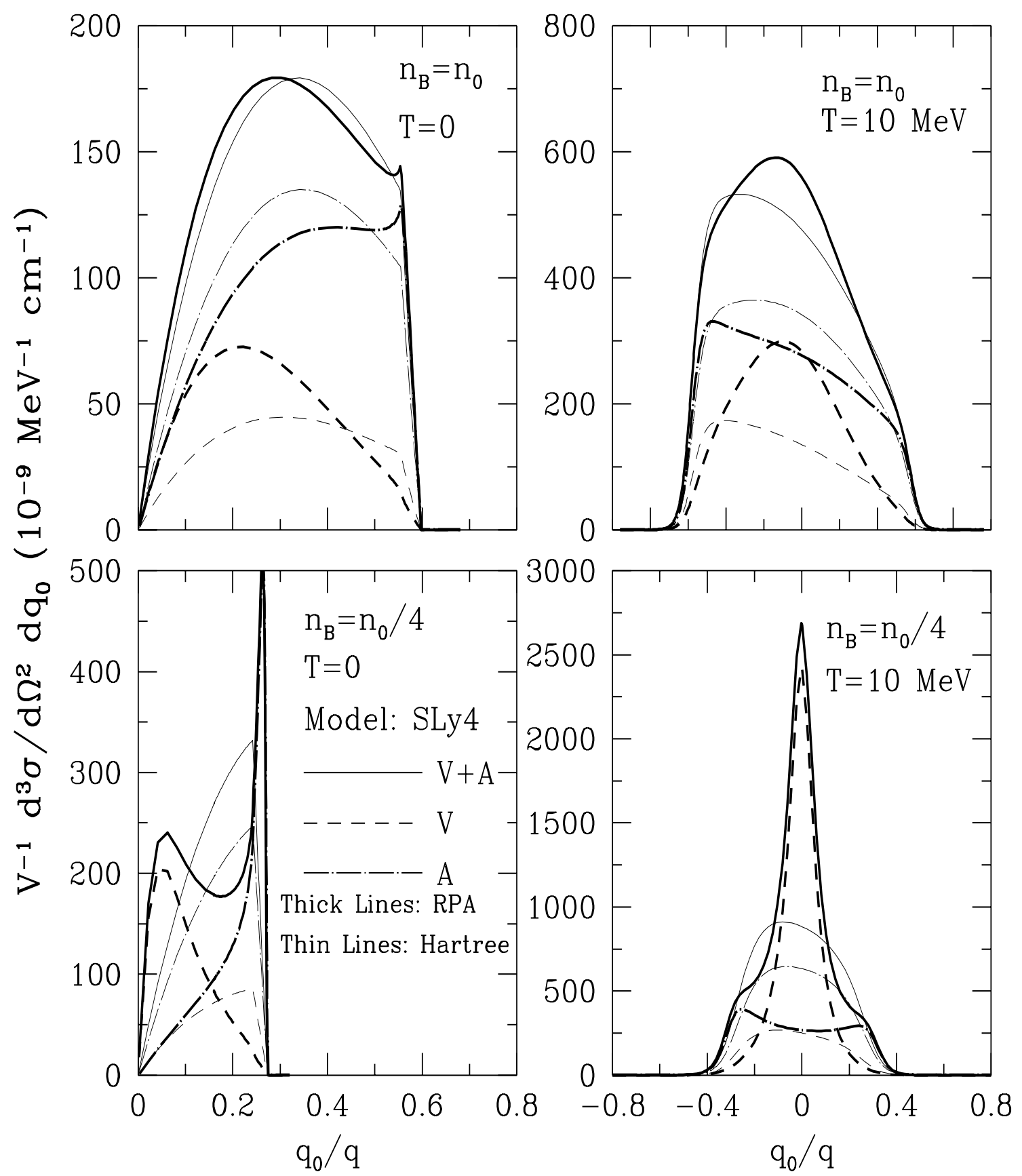

FIG. 4. 

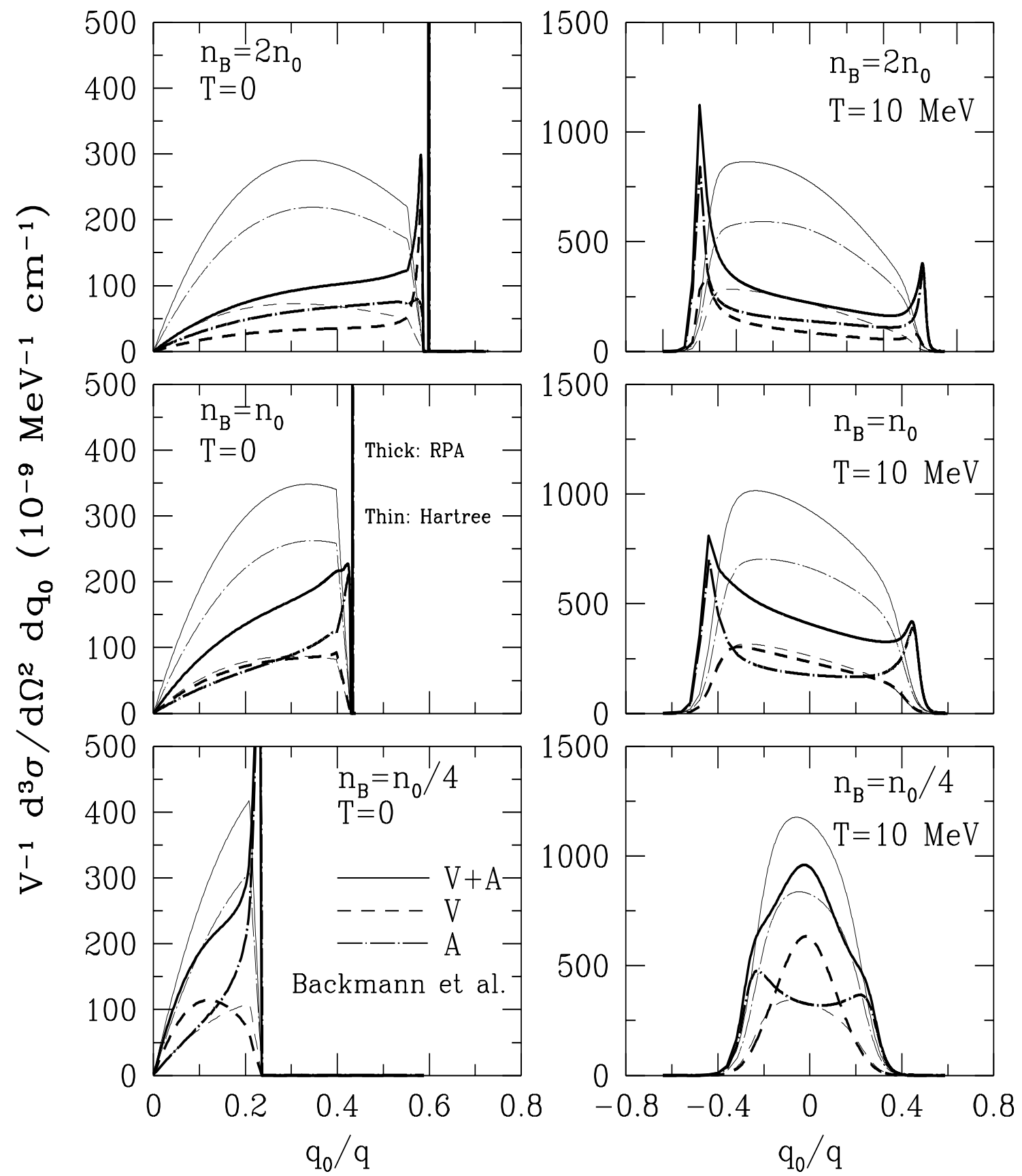

FIG. 5. 

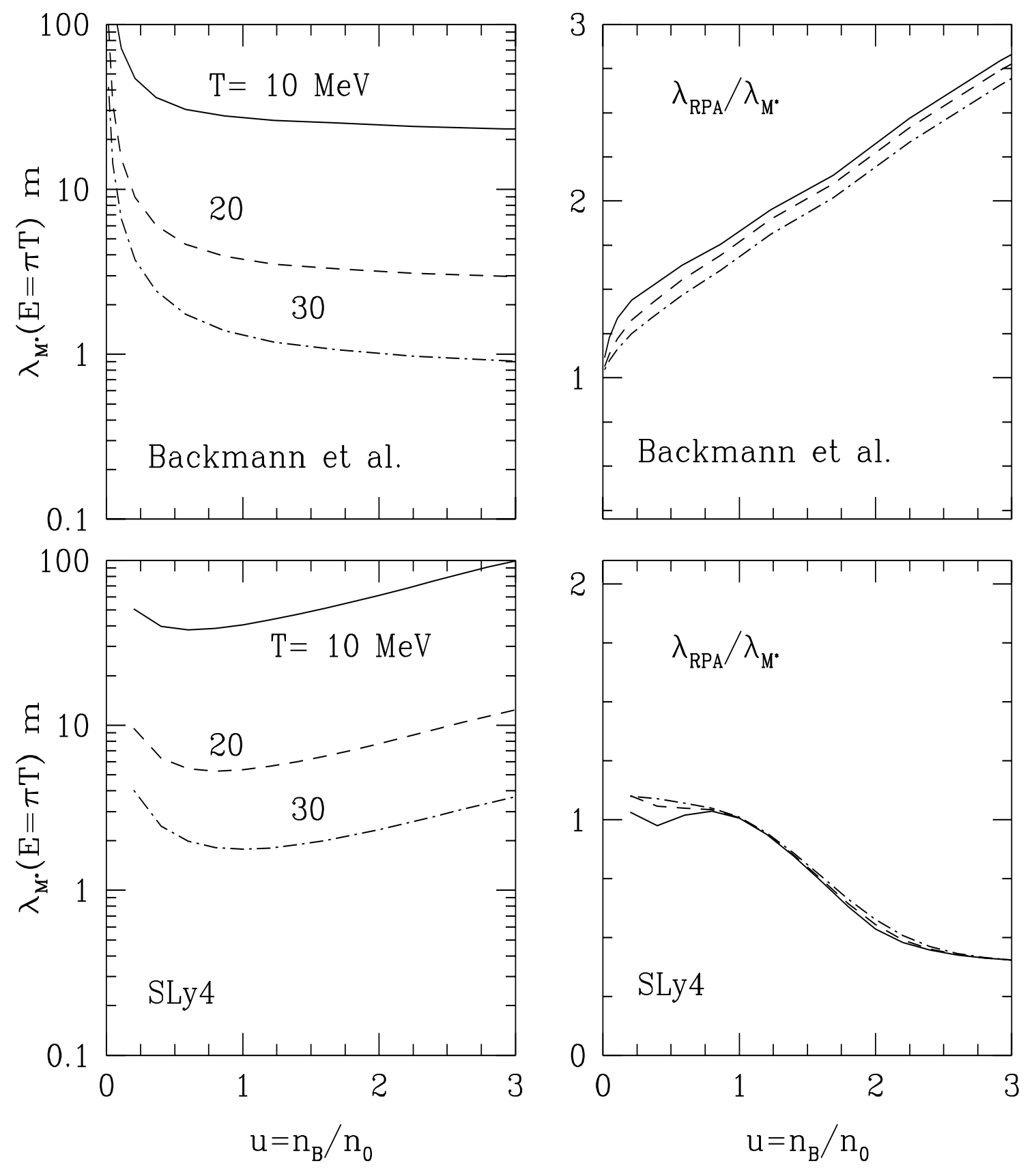

FIG. 6. 


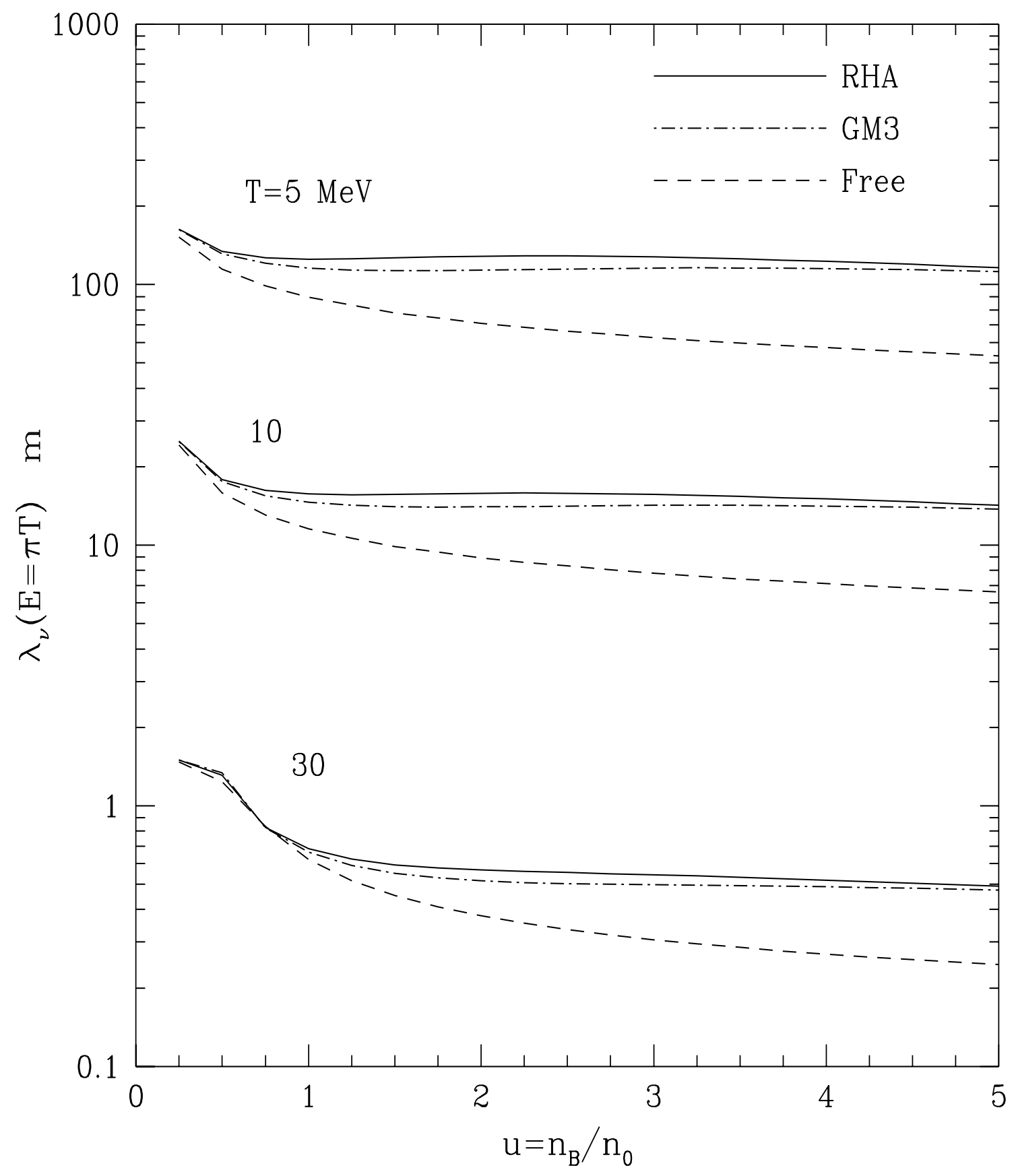

FIG. 7. 

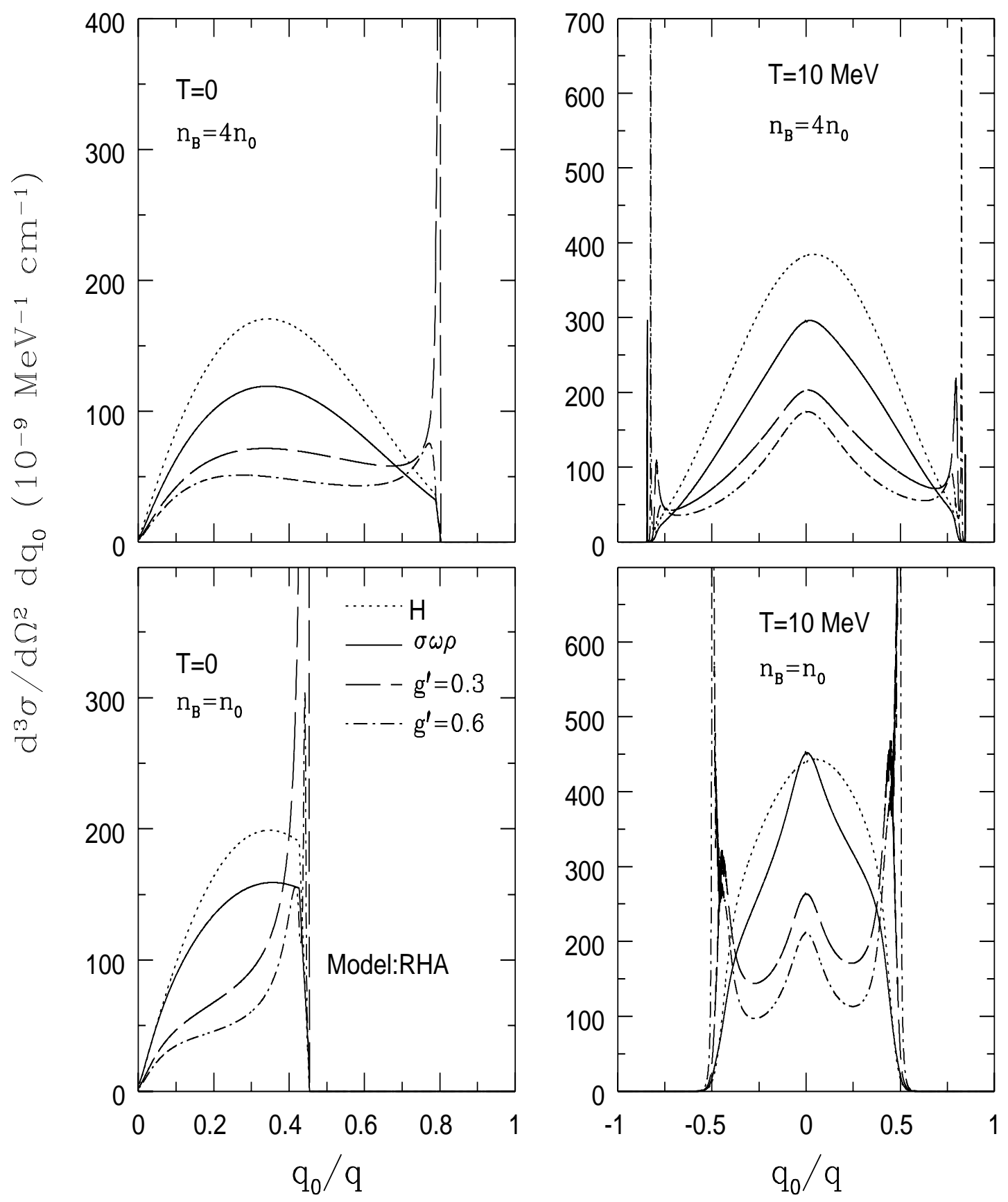

FIG. 8. 

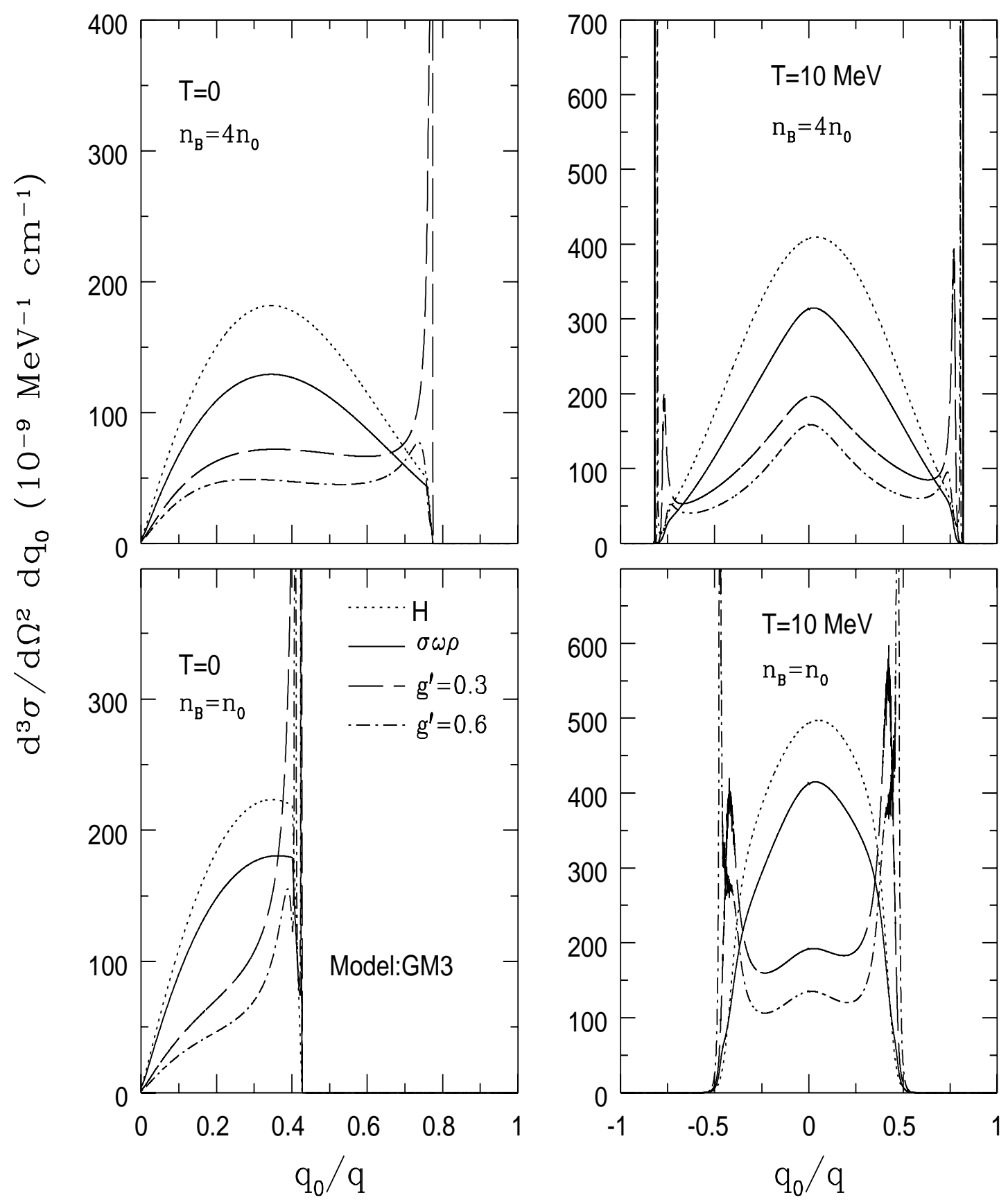

FIG. 9 . 

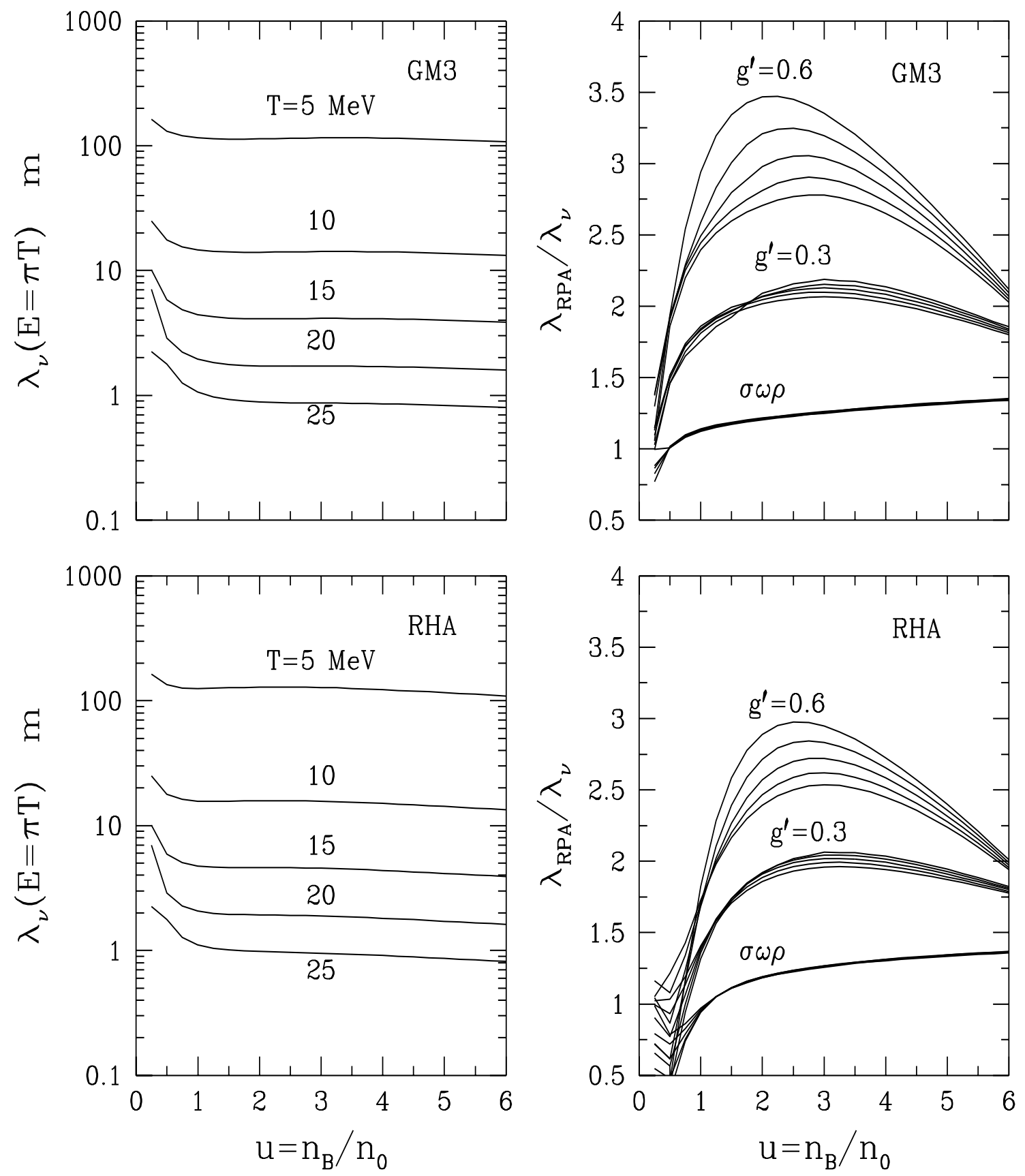

FIG. 10. 


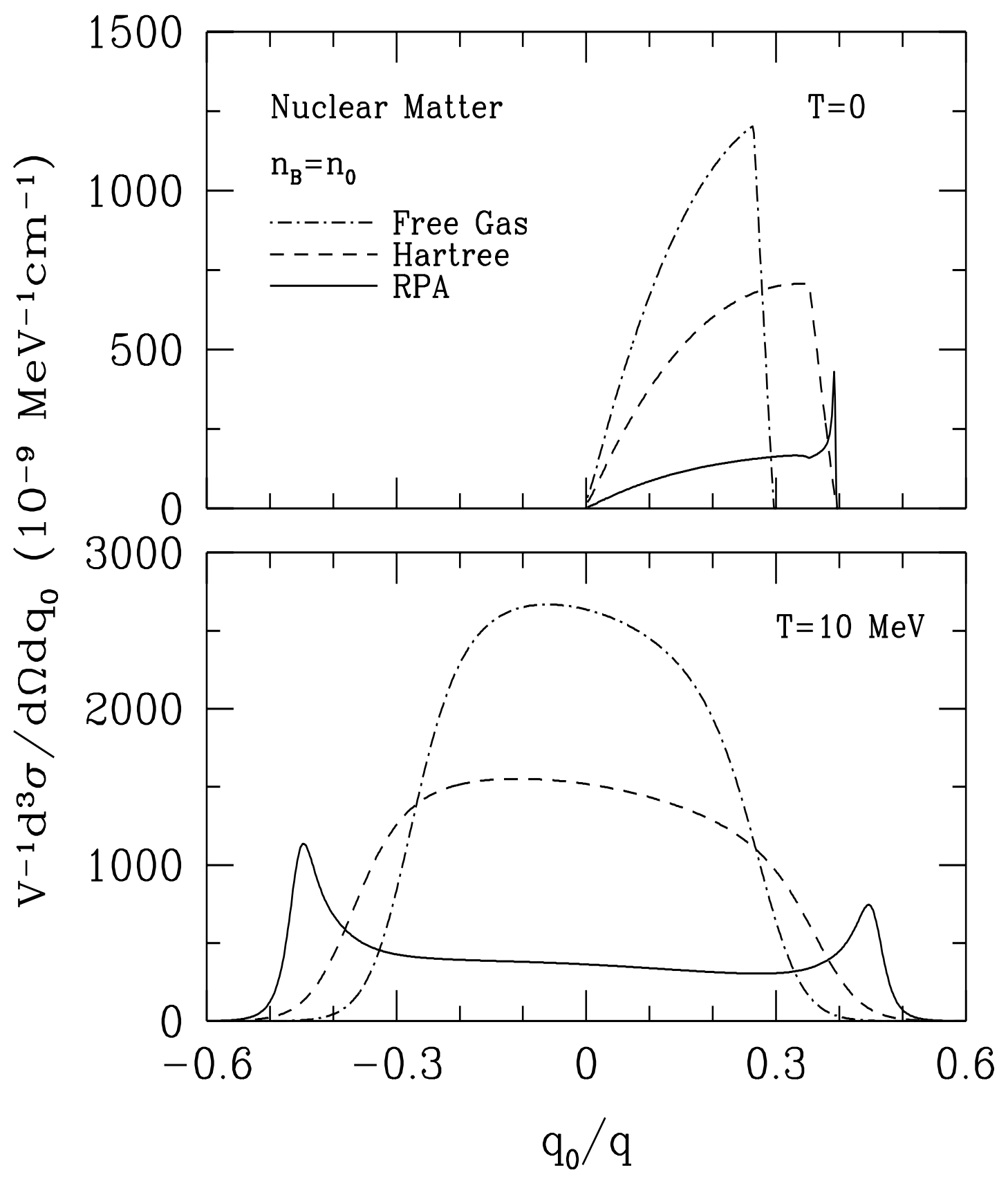

FIG. 11. 

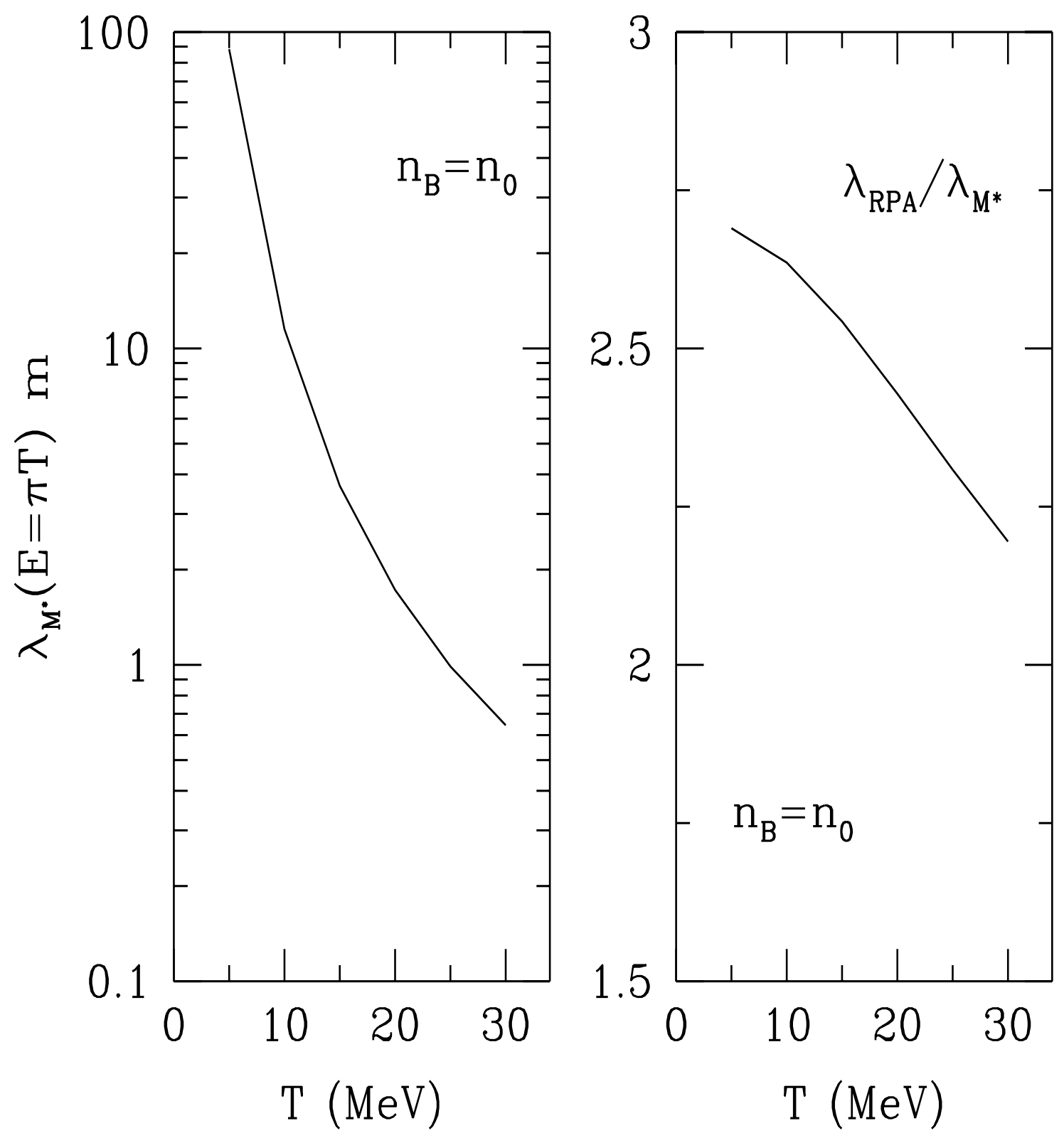

FIG. 12. 


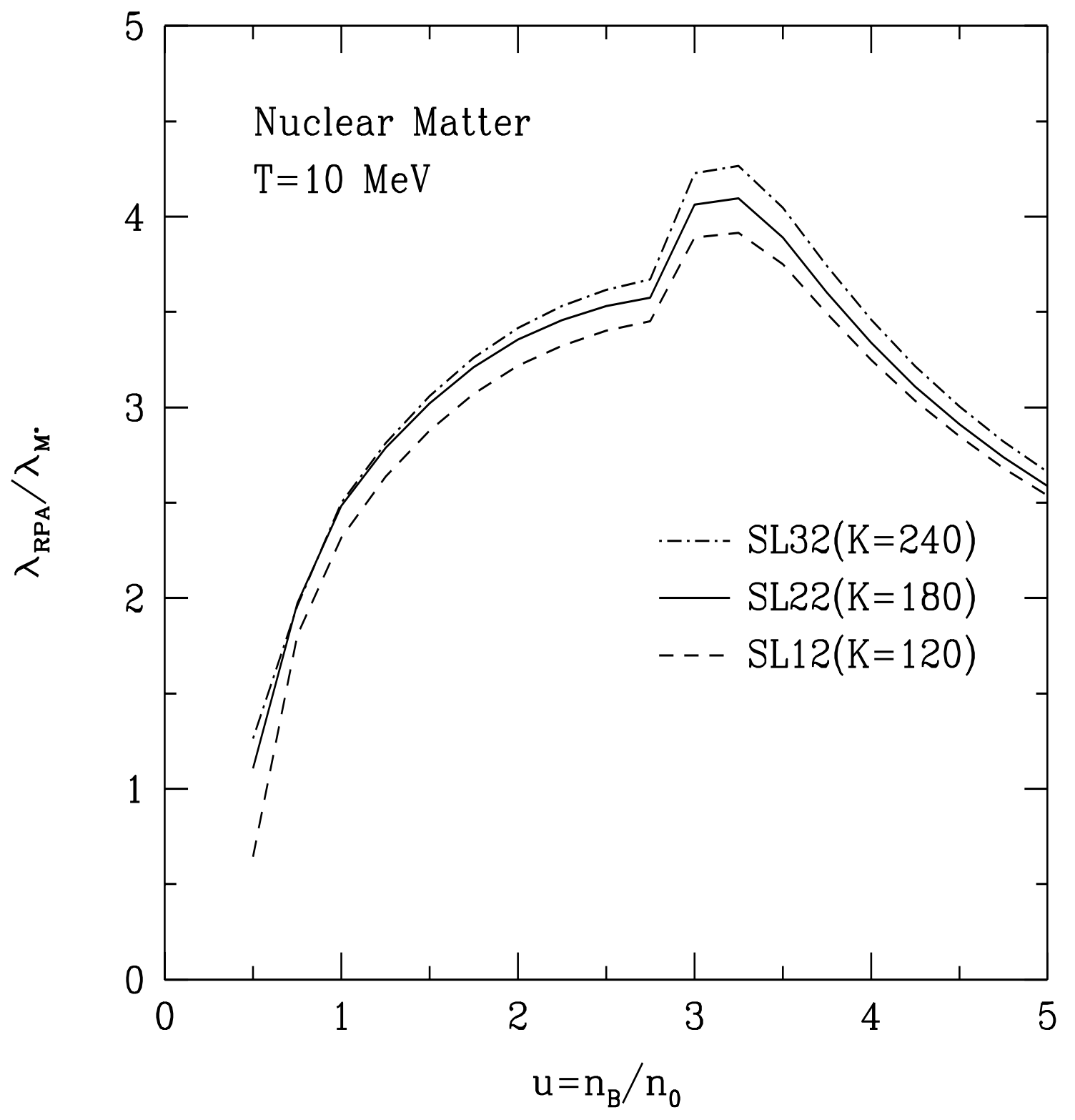

FIG. 13. 

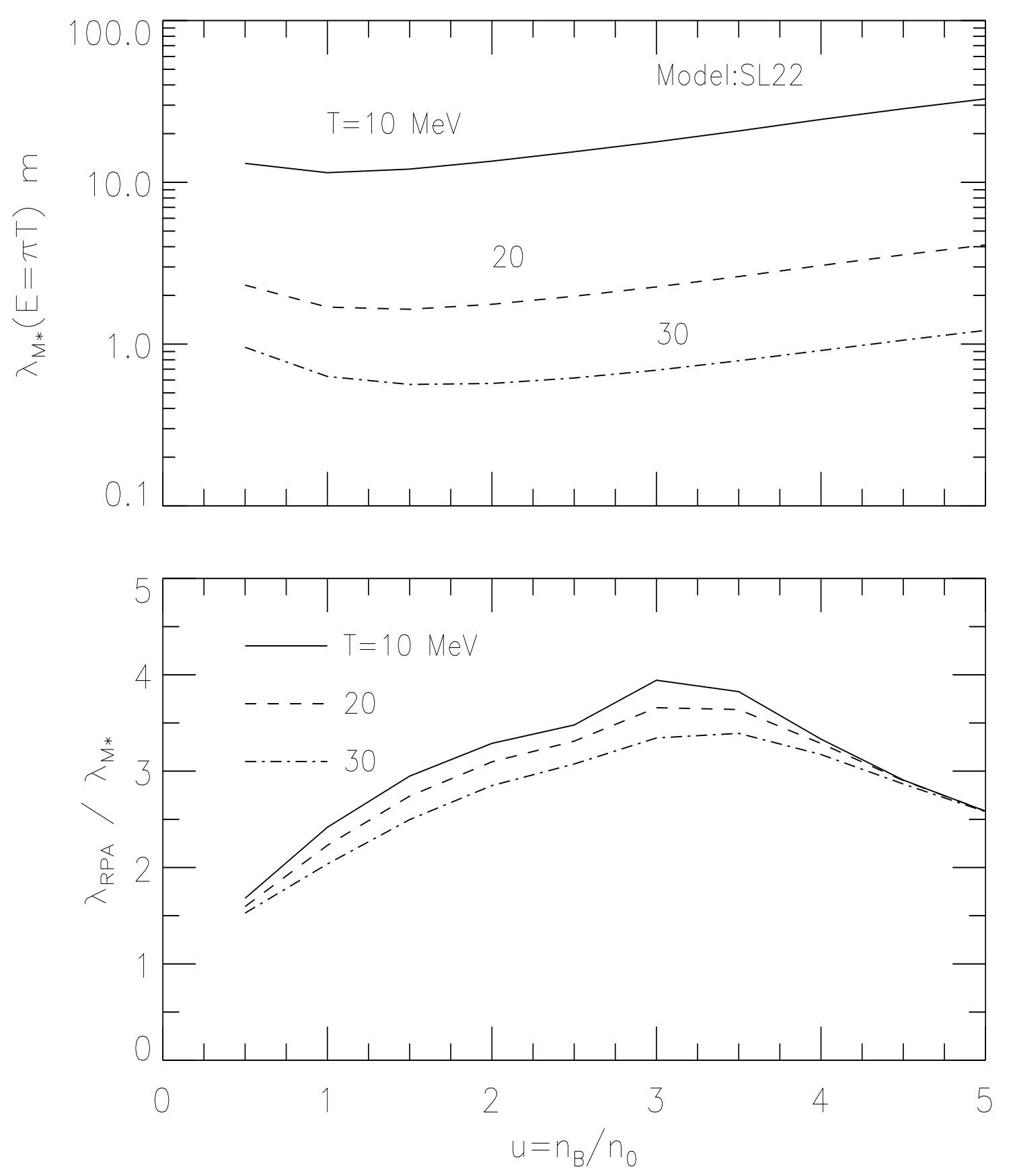

FIG. 14. 

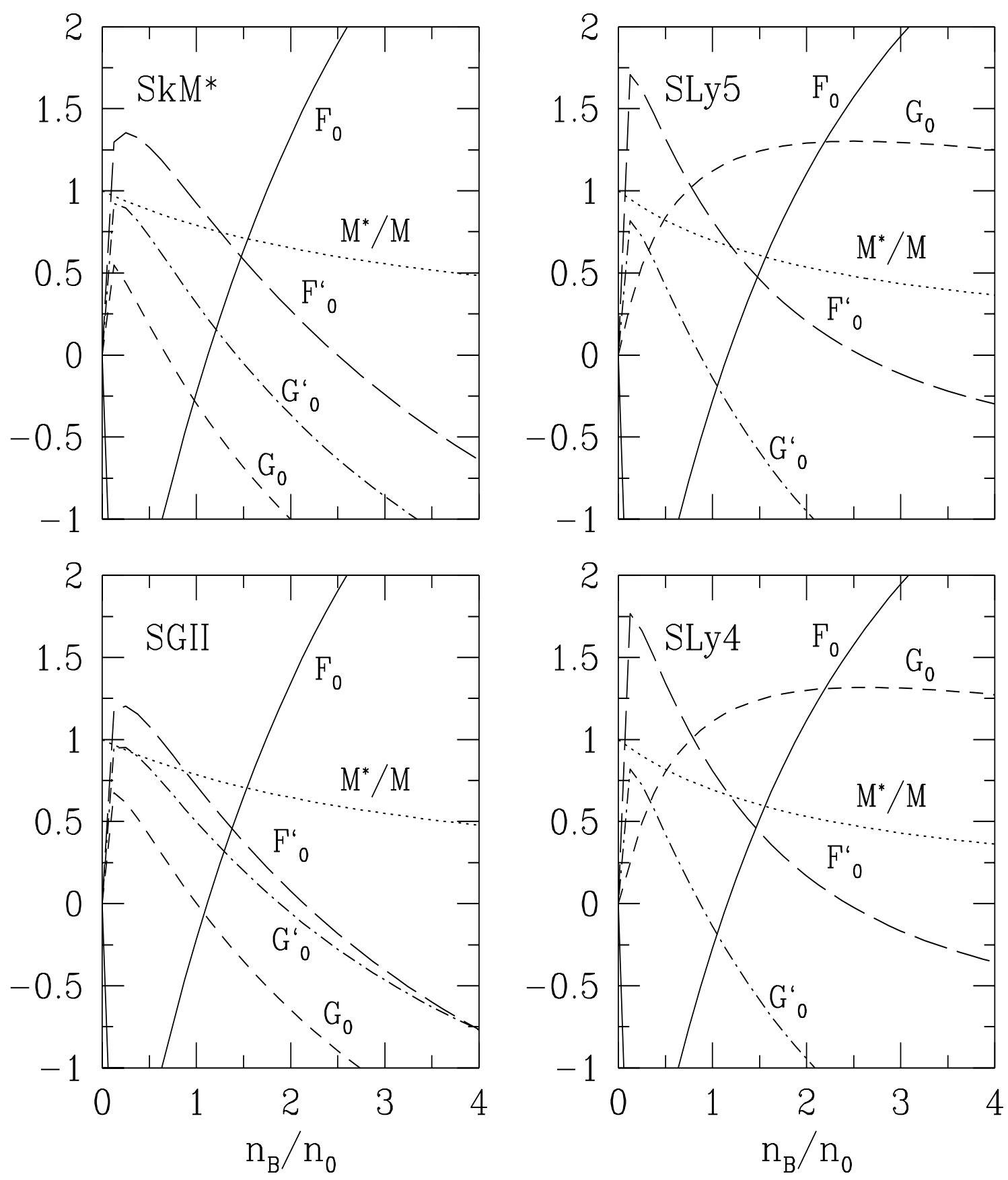

FIG. 15. 

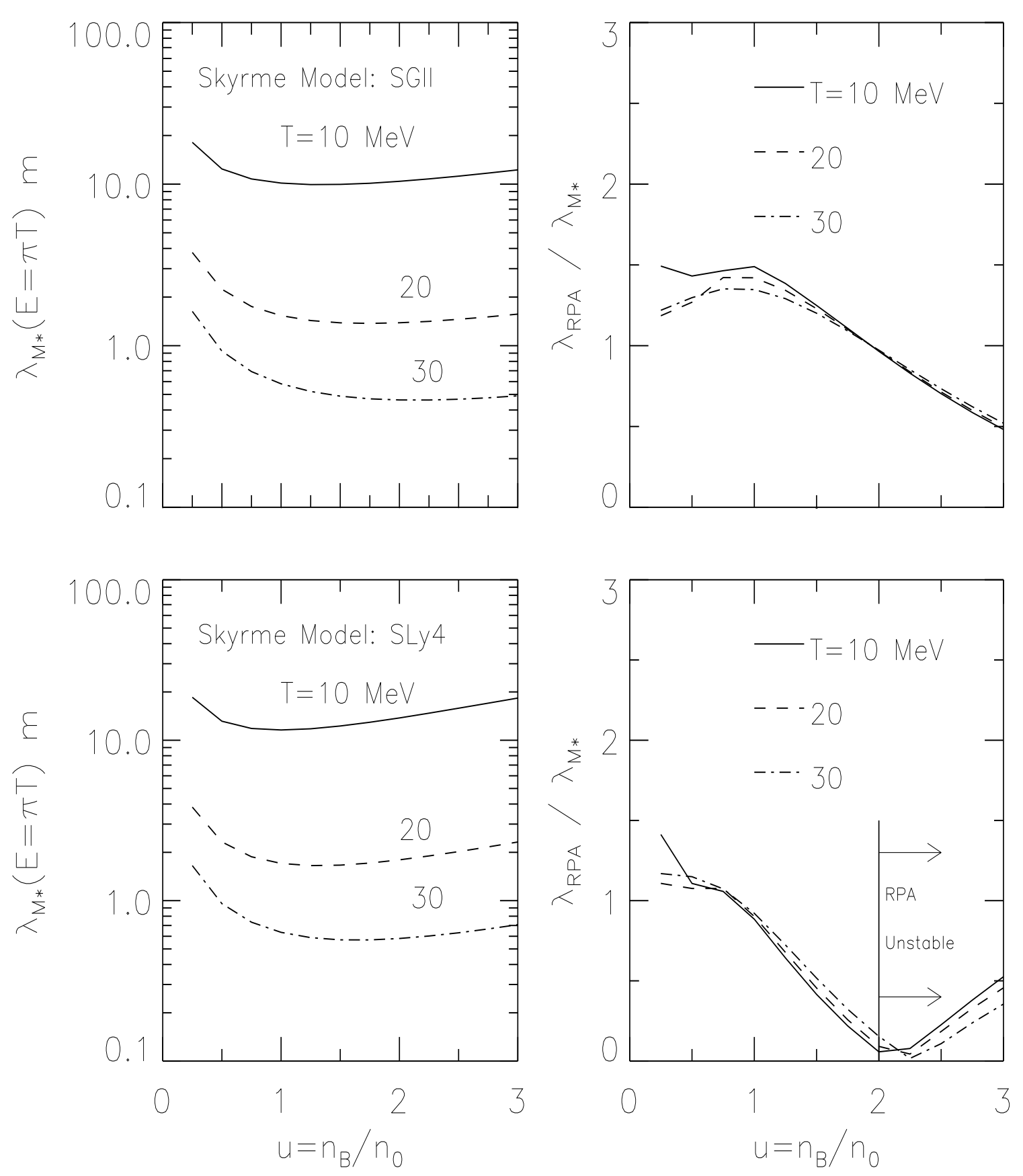

FIG. 16. 

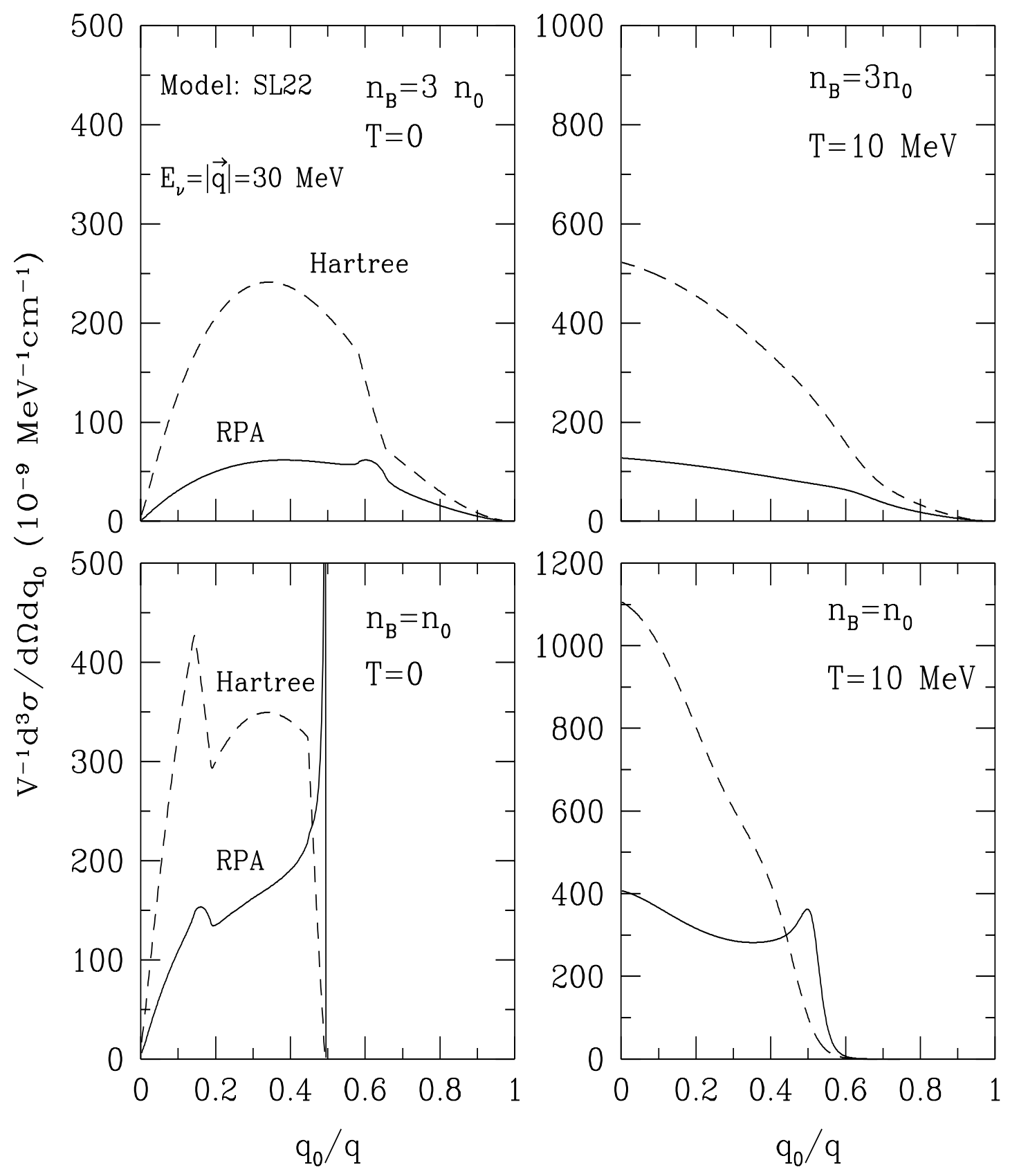

FIG. 17. 


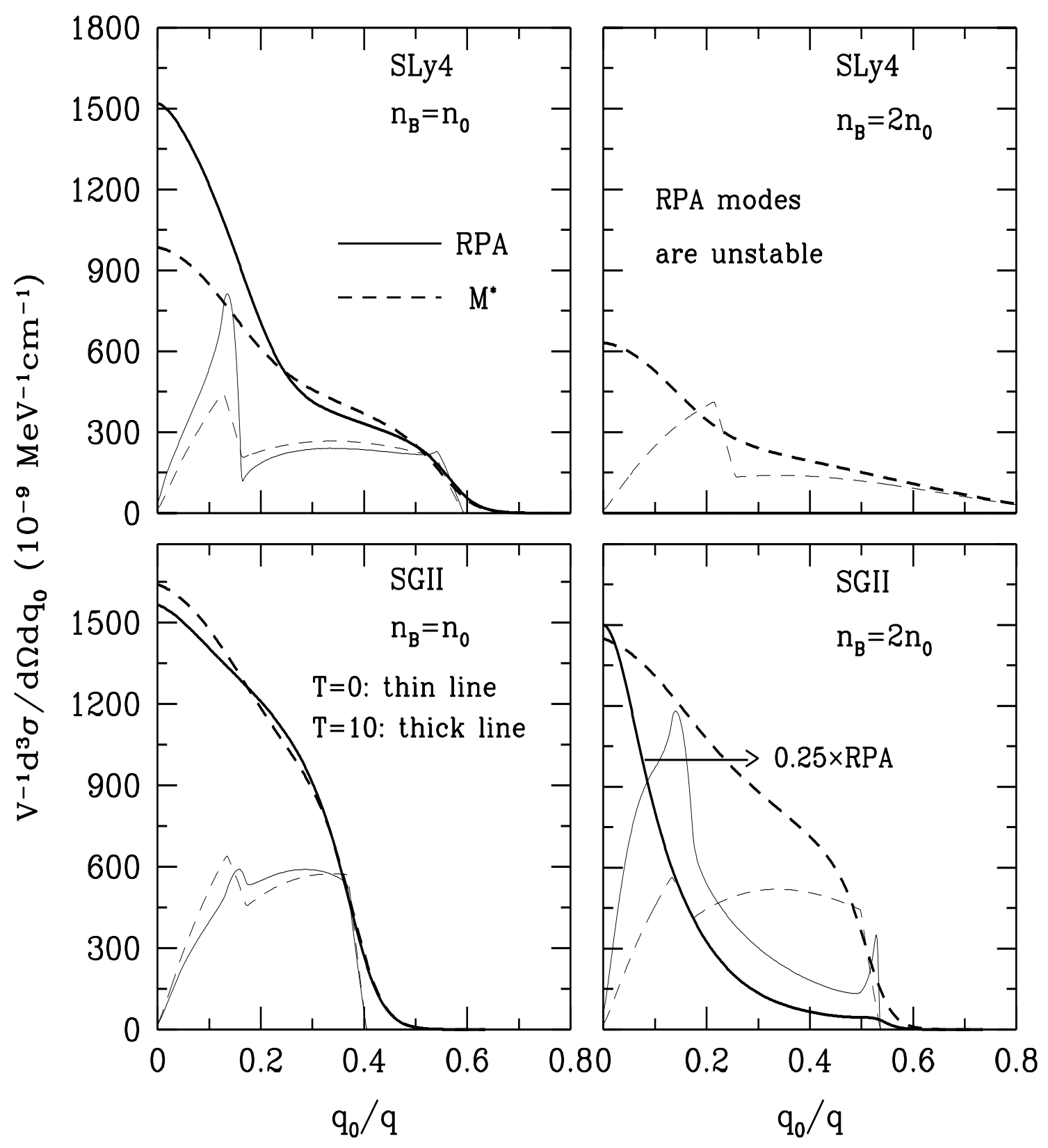

FIG. 18. 

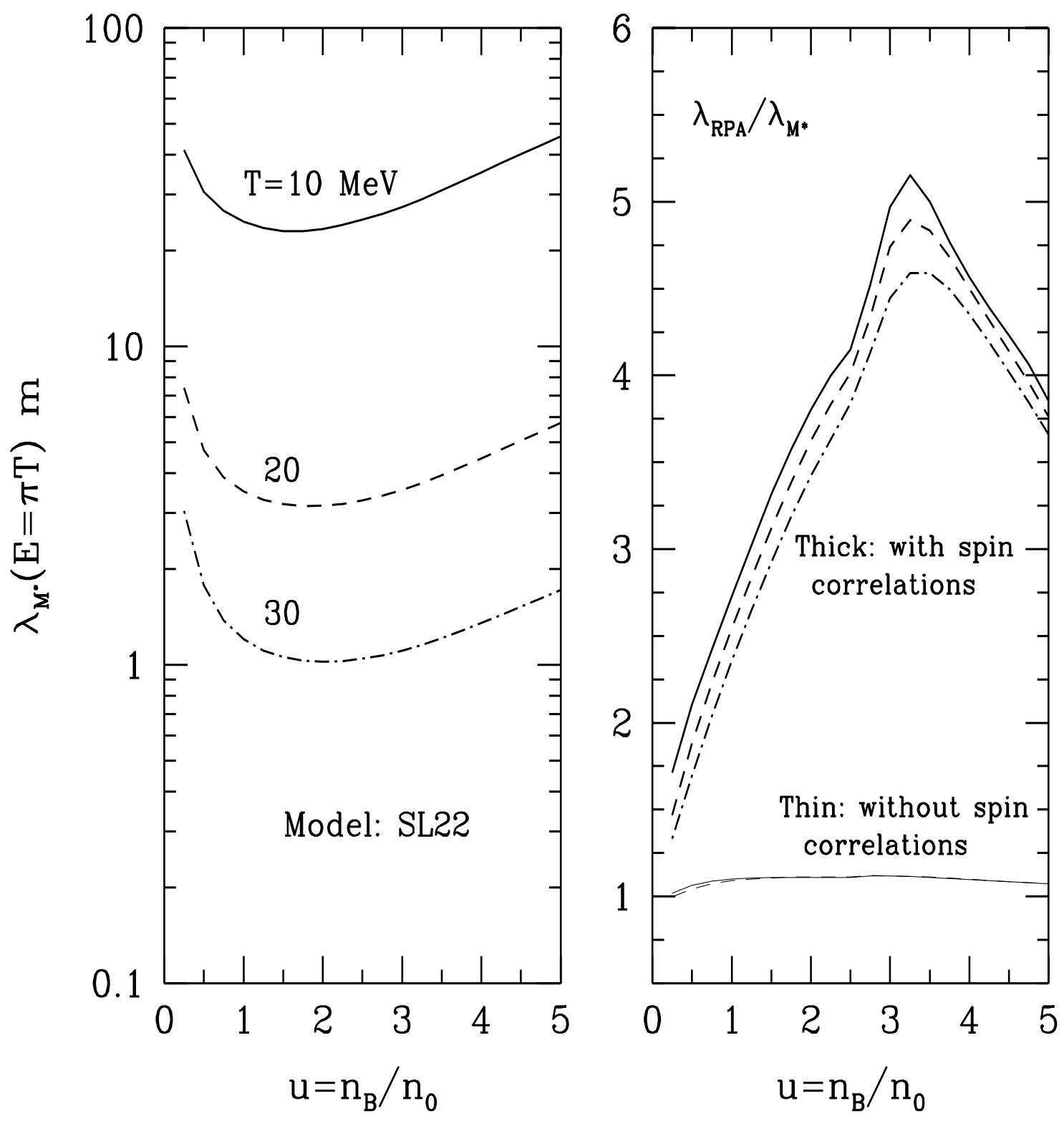

FIG. 19. 


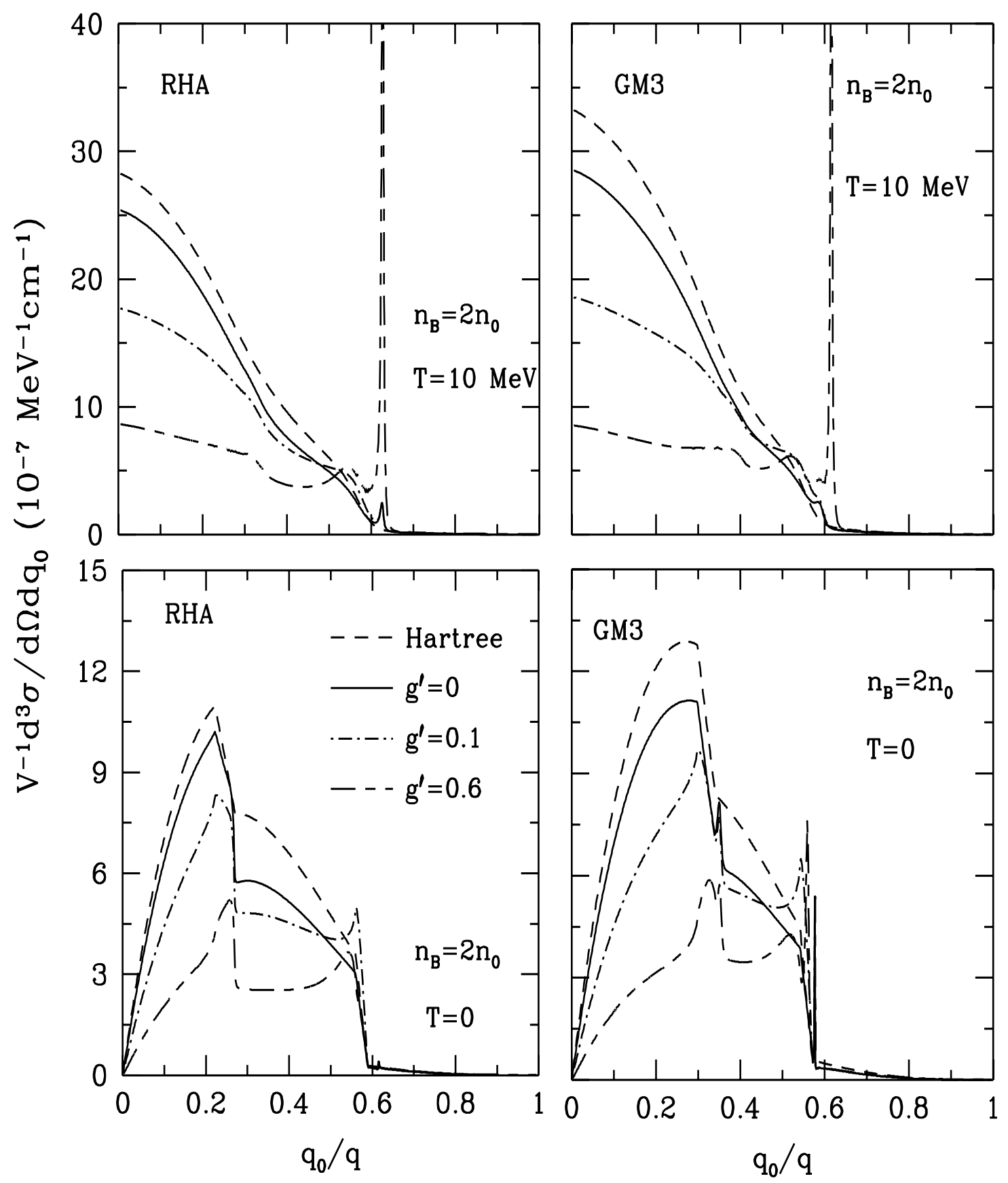

FIG. 20. 

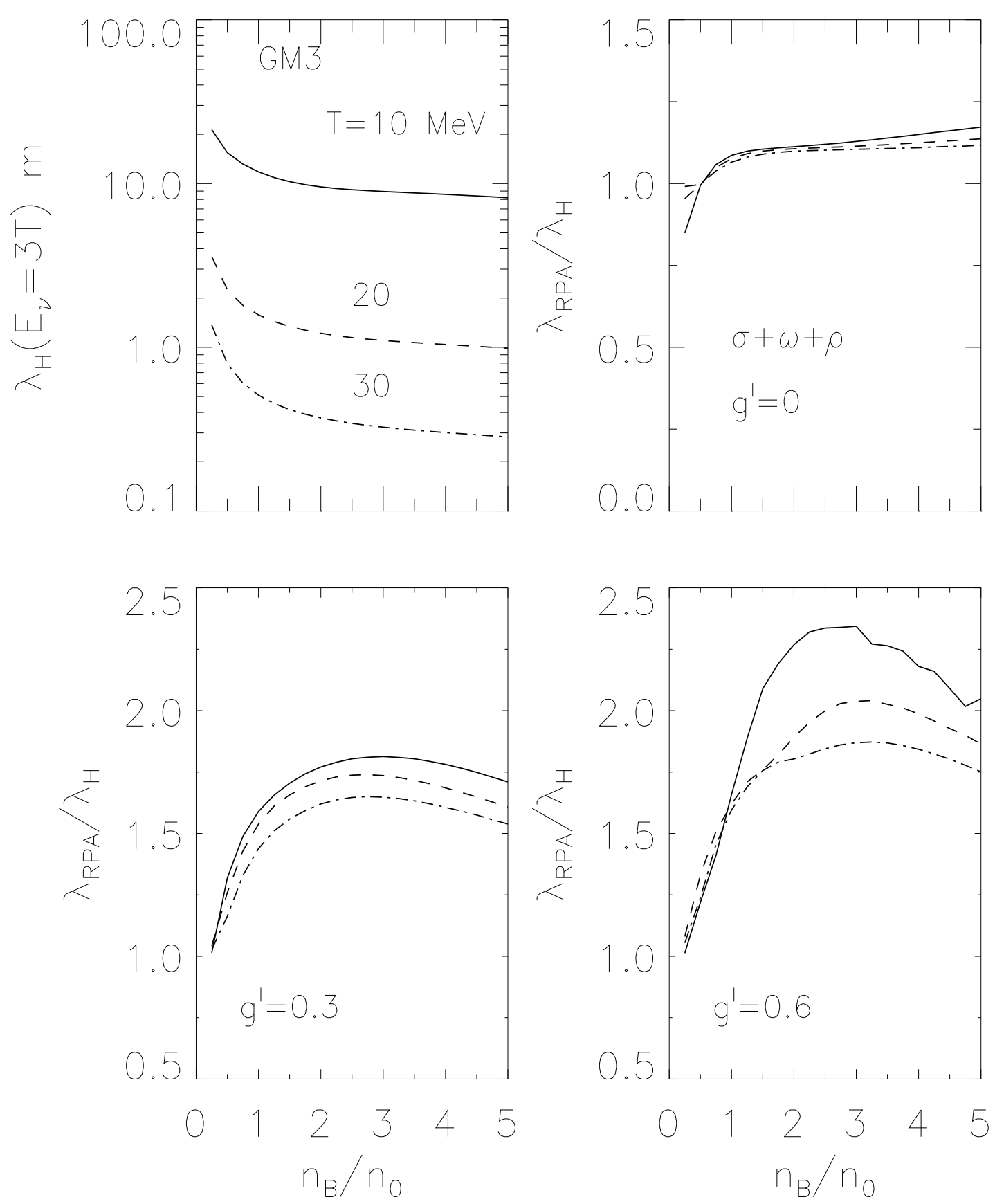

FIG. 21. 


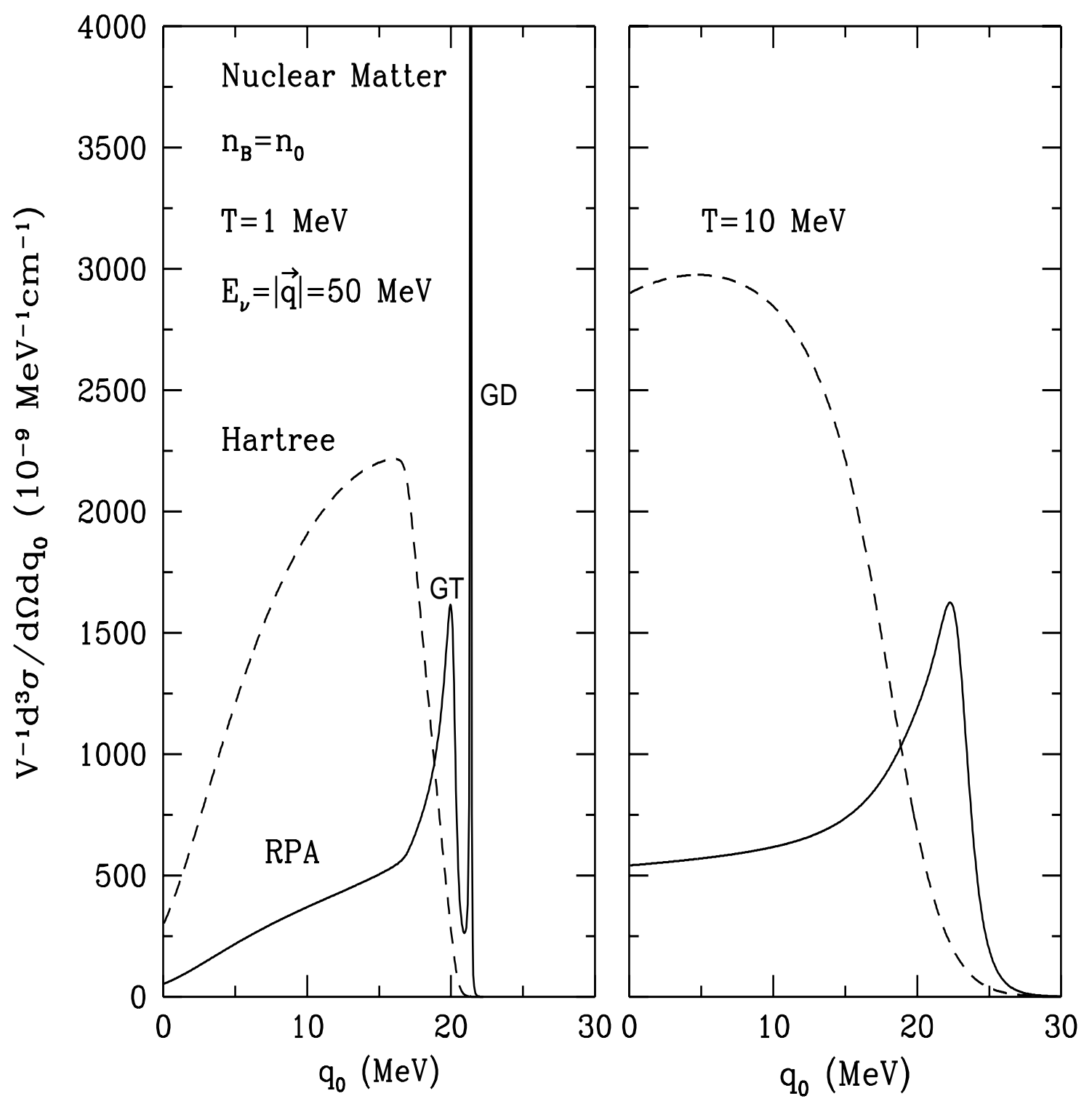

FIG. 22. 


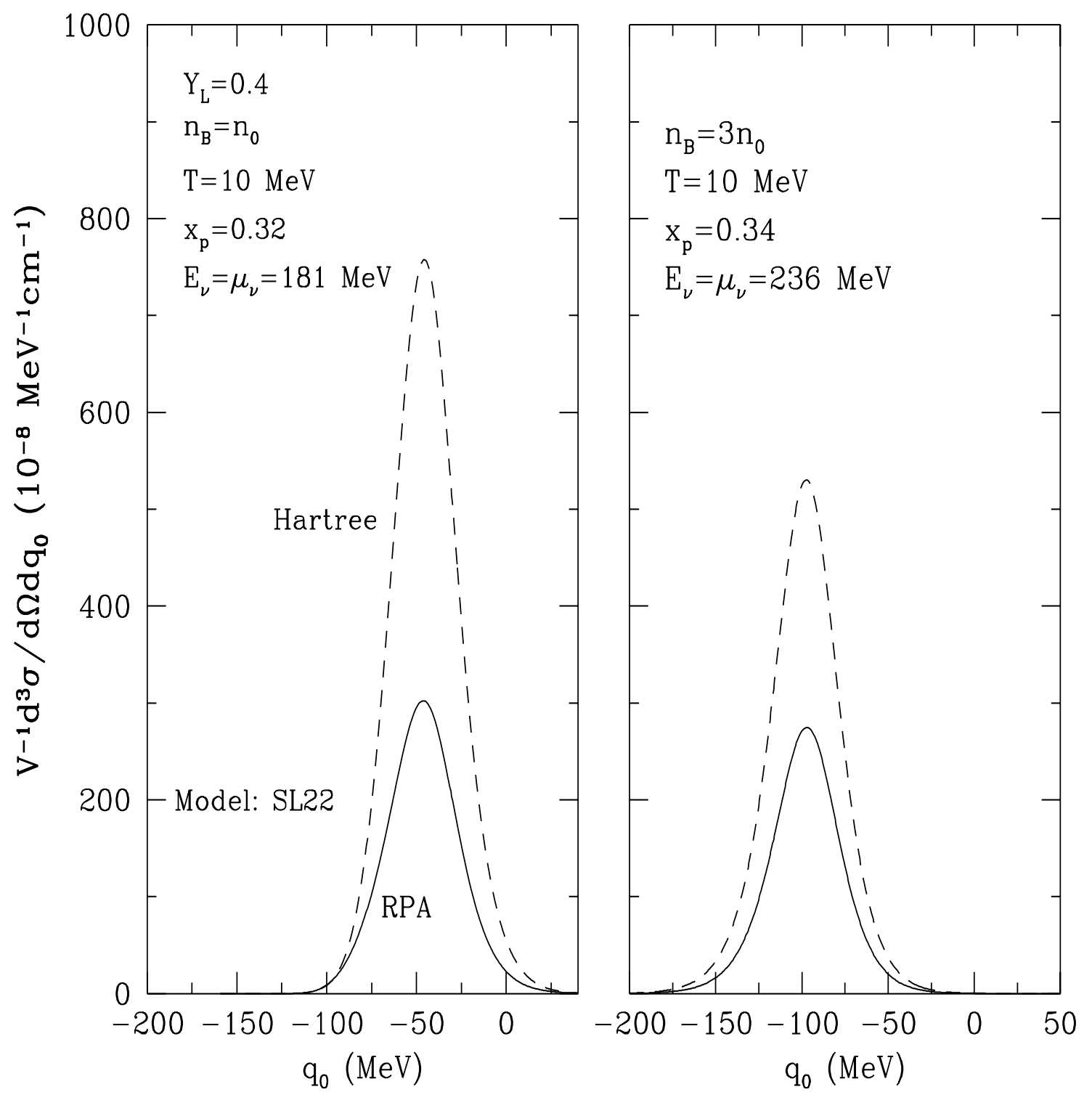

FIG. 23. 


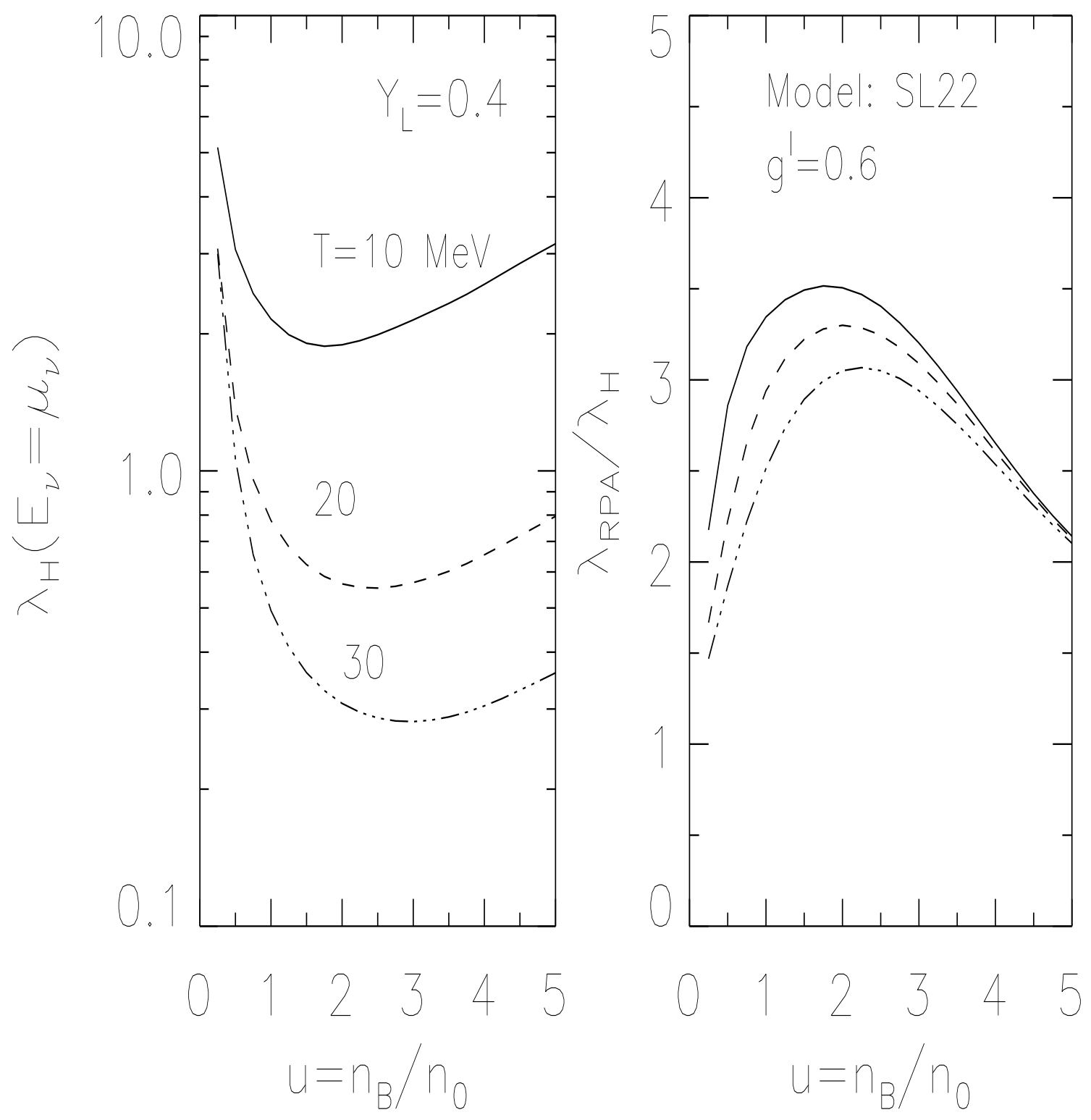

FIG. 24. 


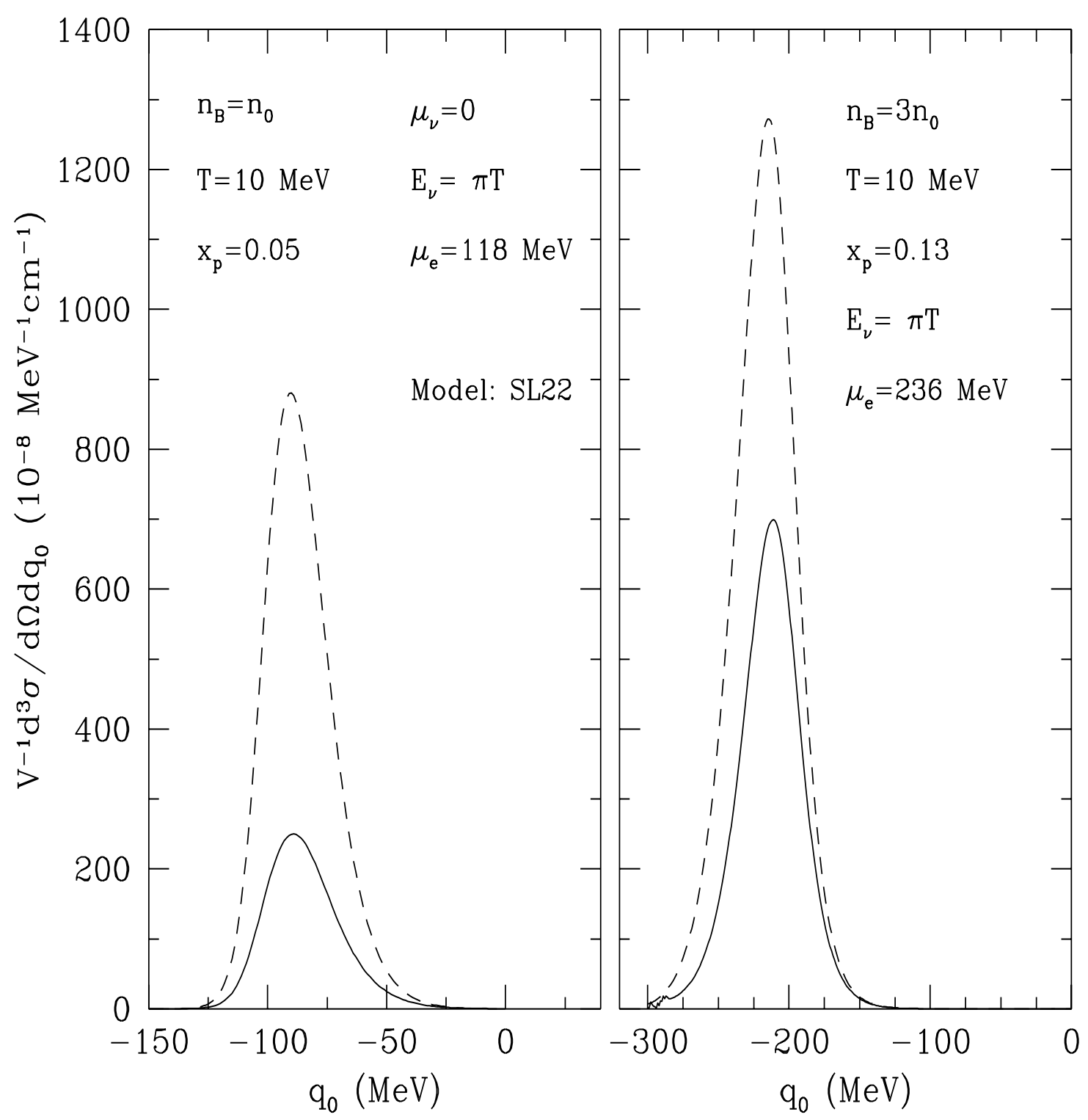

FIG. 25. 

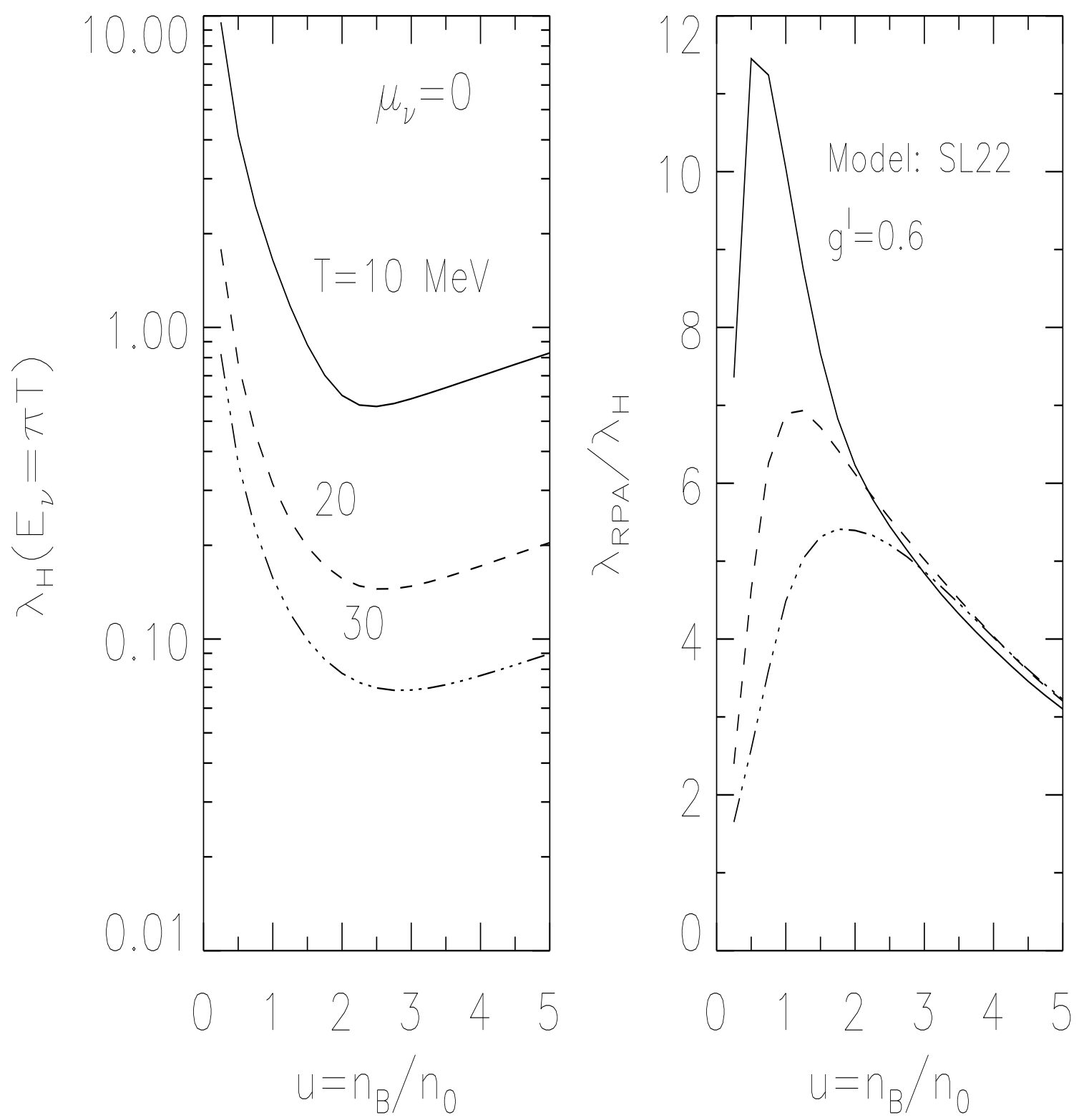

FIG. 26. 


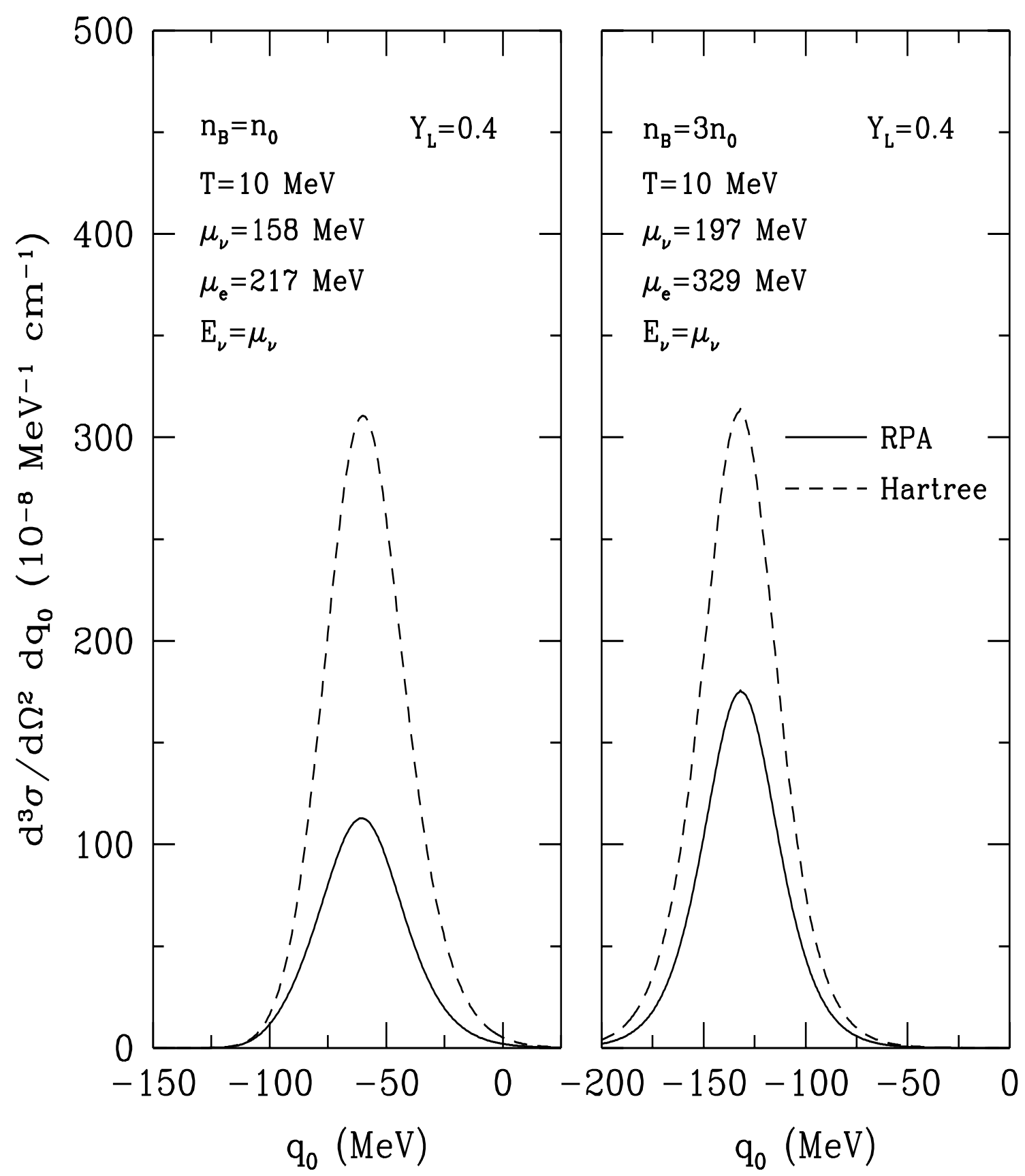

FIG. 27. 

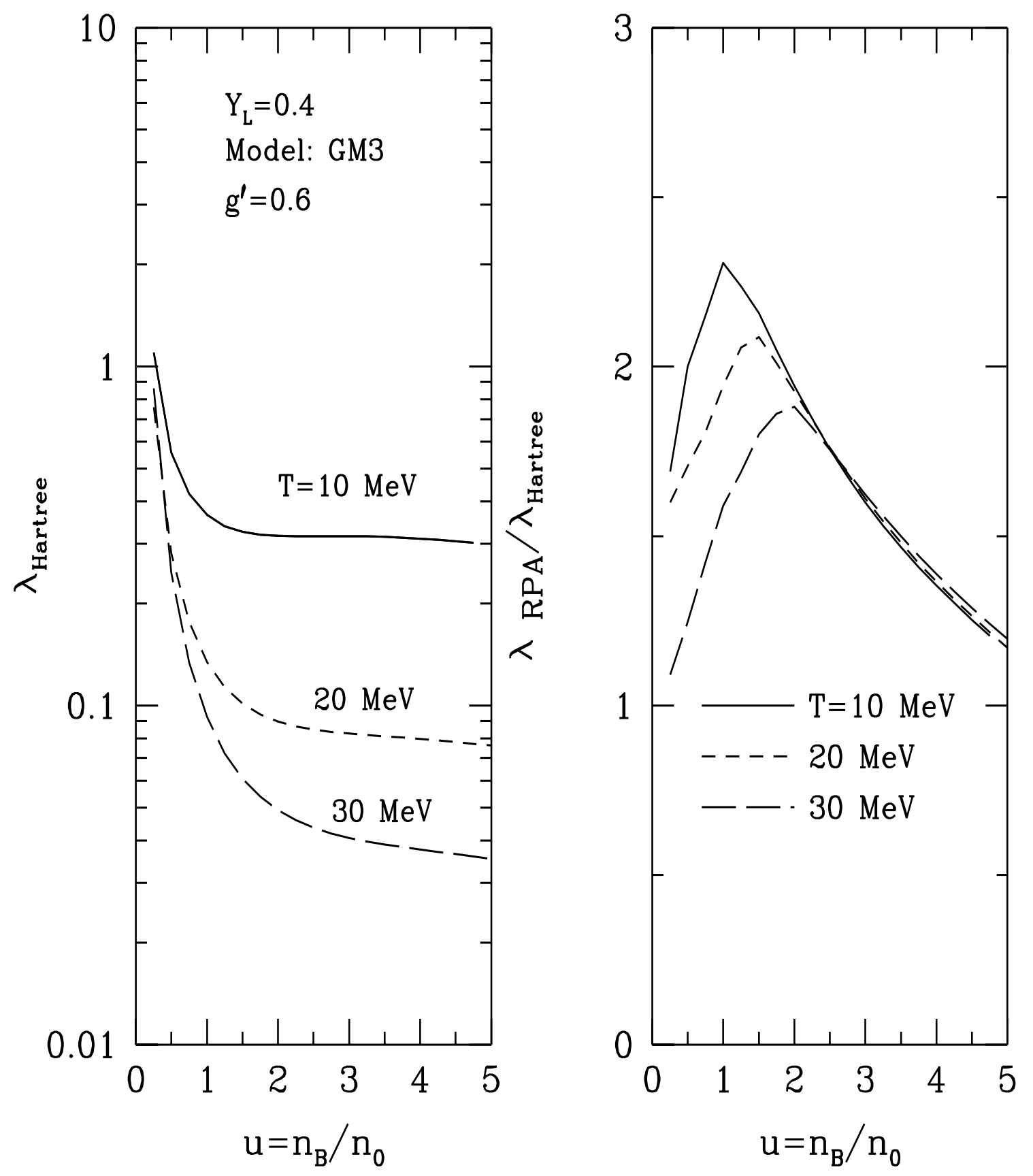

FIG. 28. 


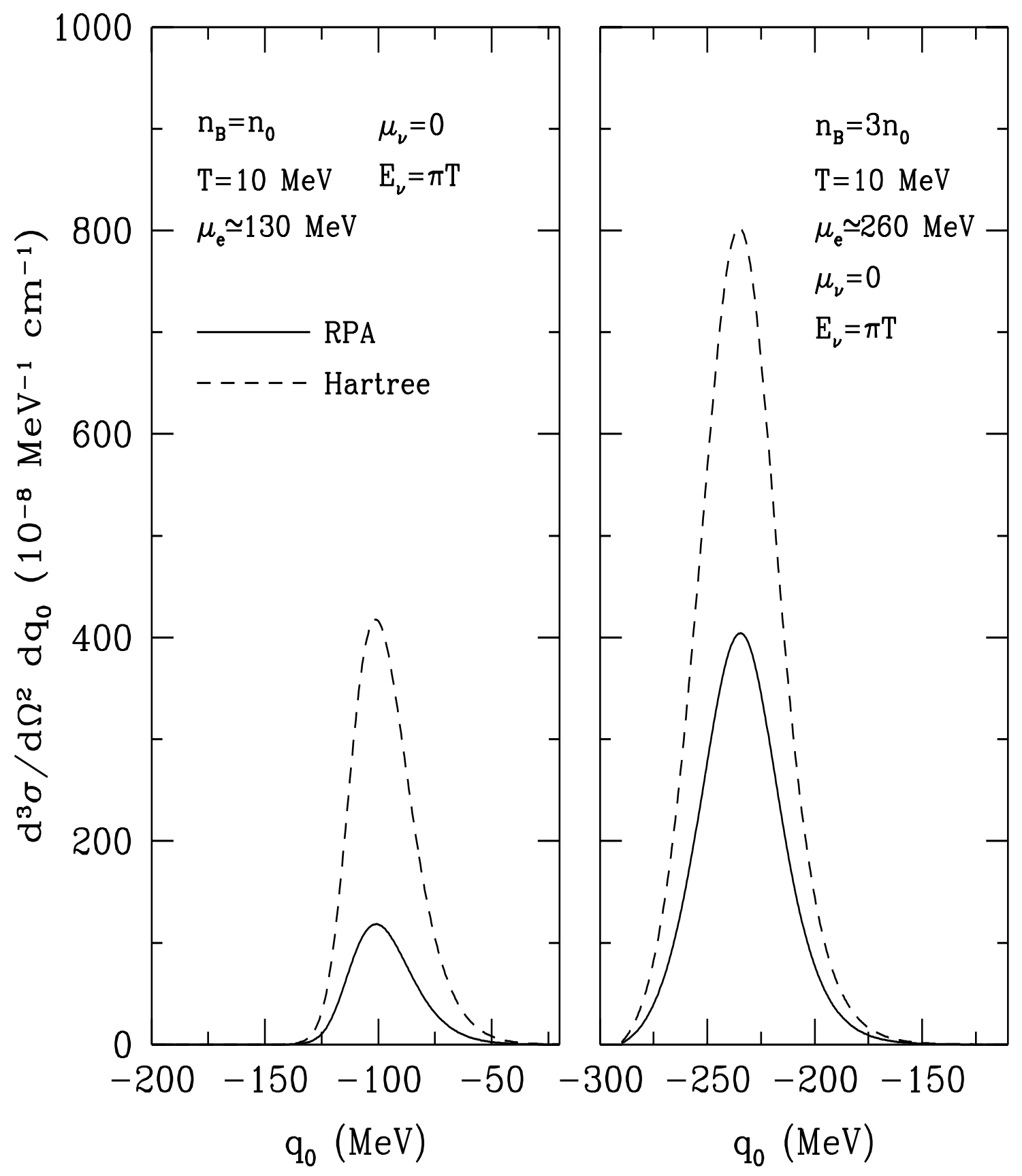

FIG. 29. 

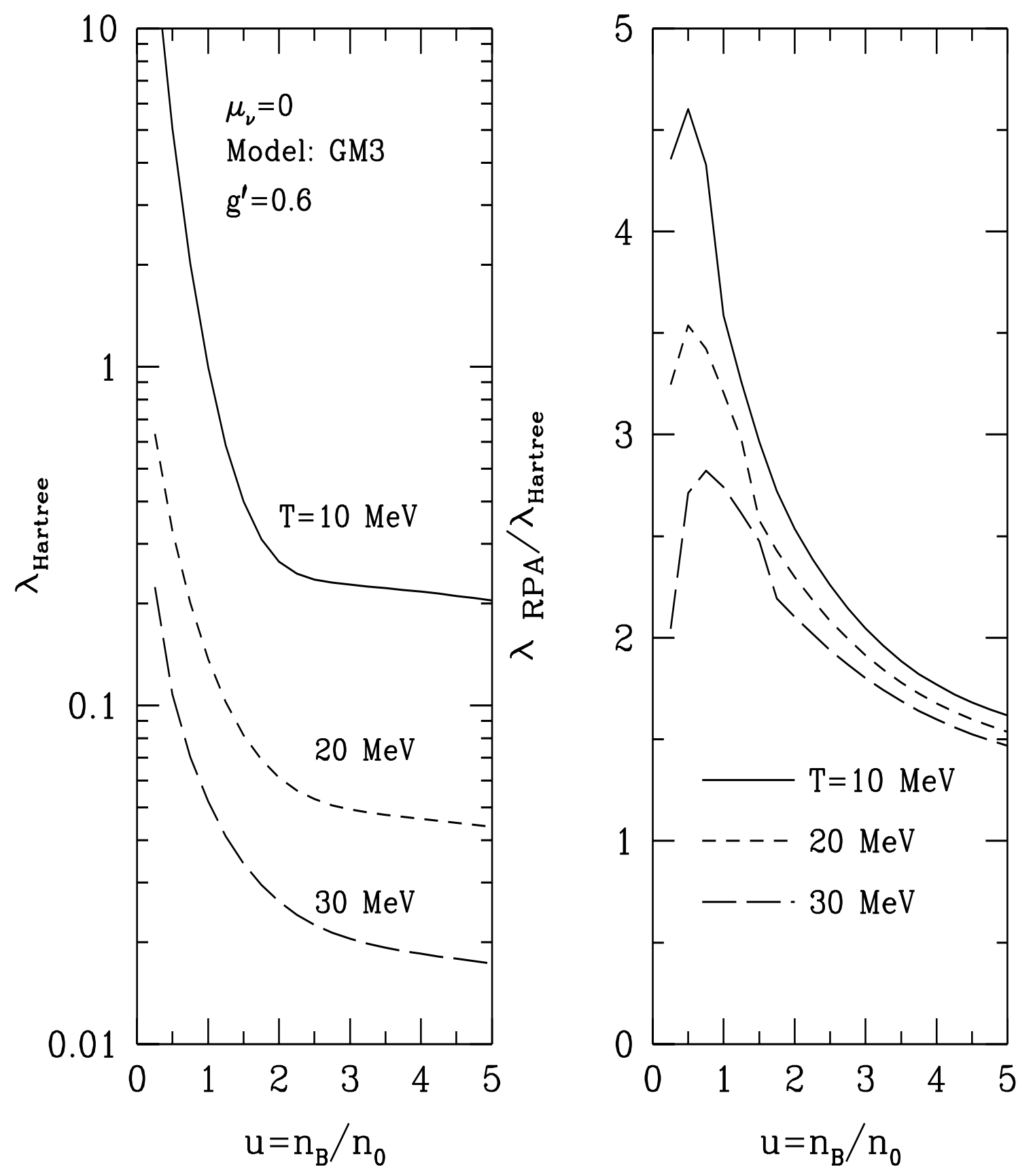

FIG. 30 . 
GM3

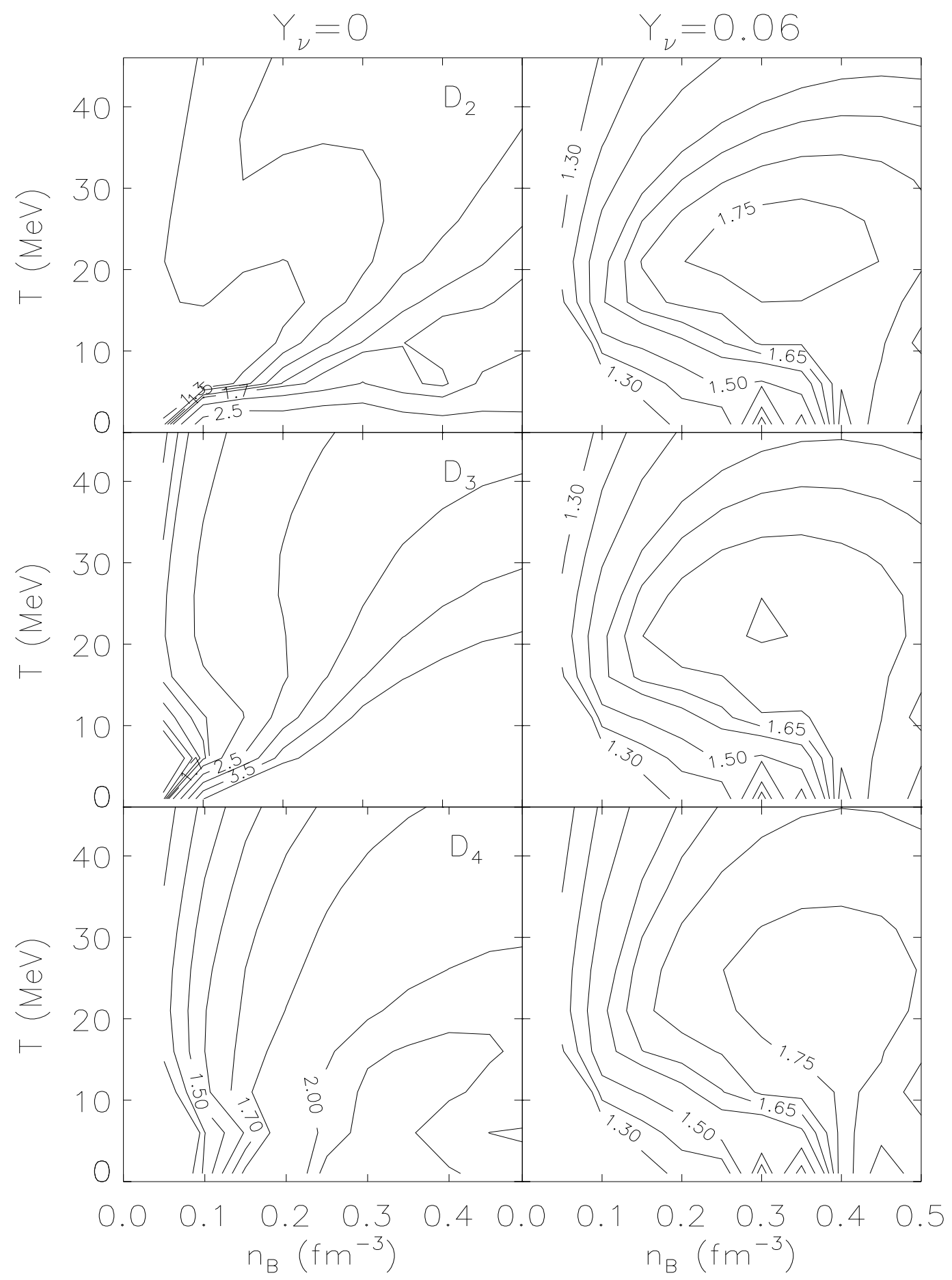

FIG. 31. 


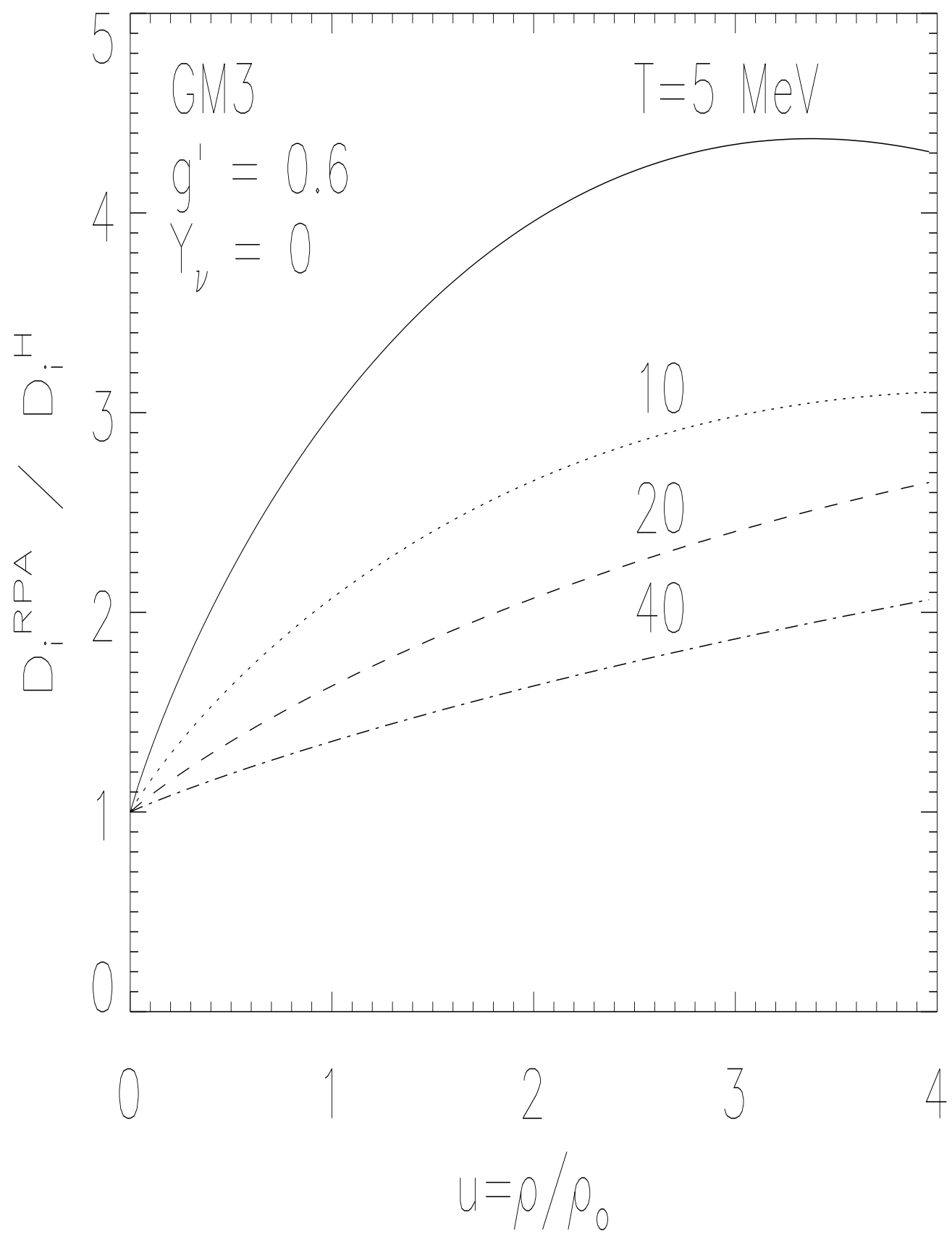

FIG. 32. 

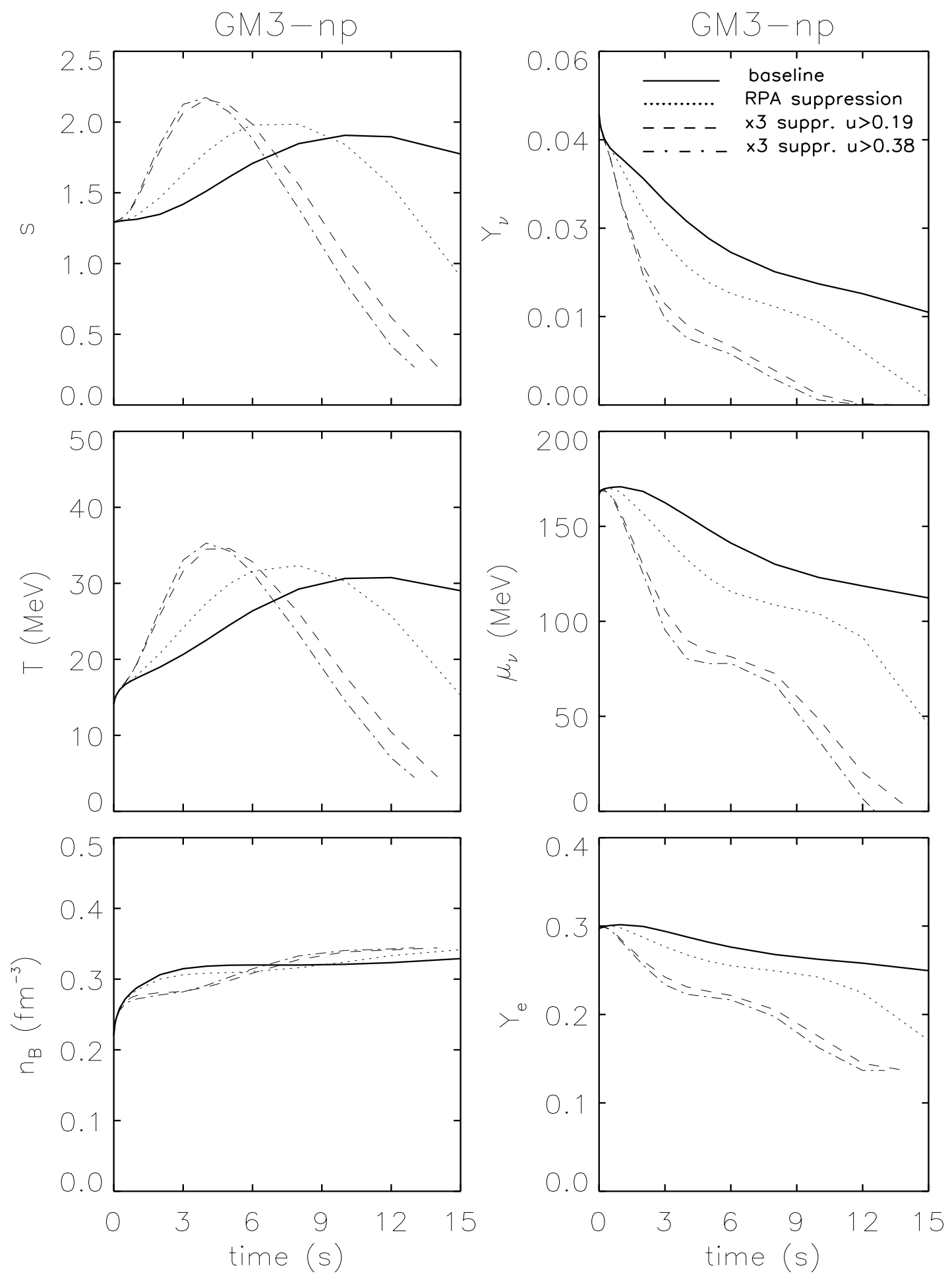

FIG. 33. 

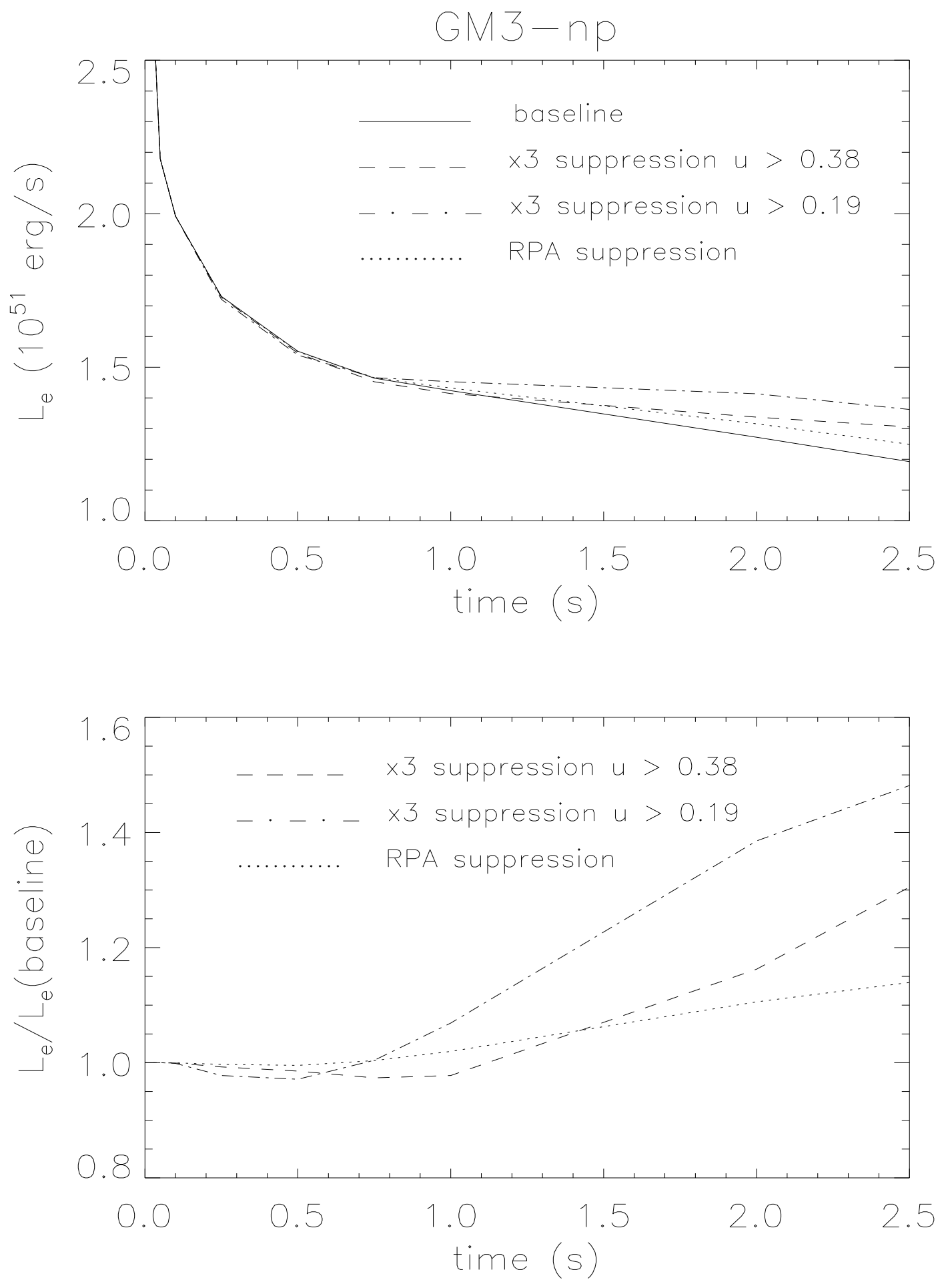

FIG. 34 . 


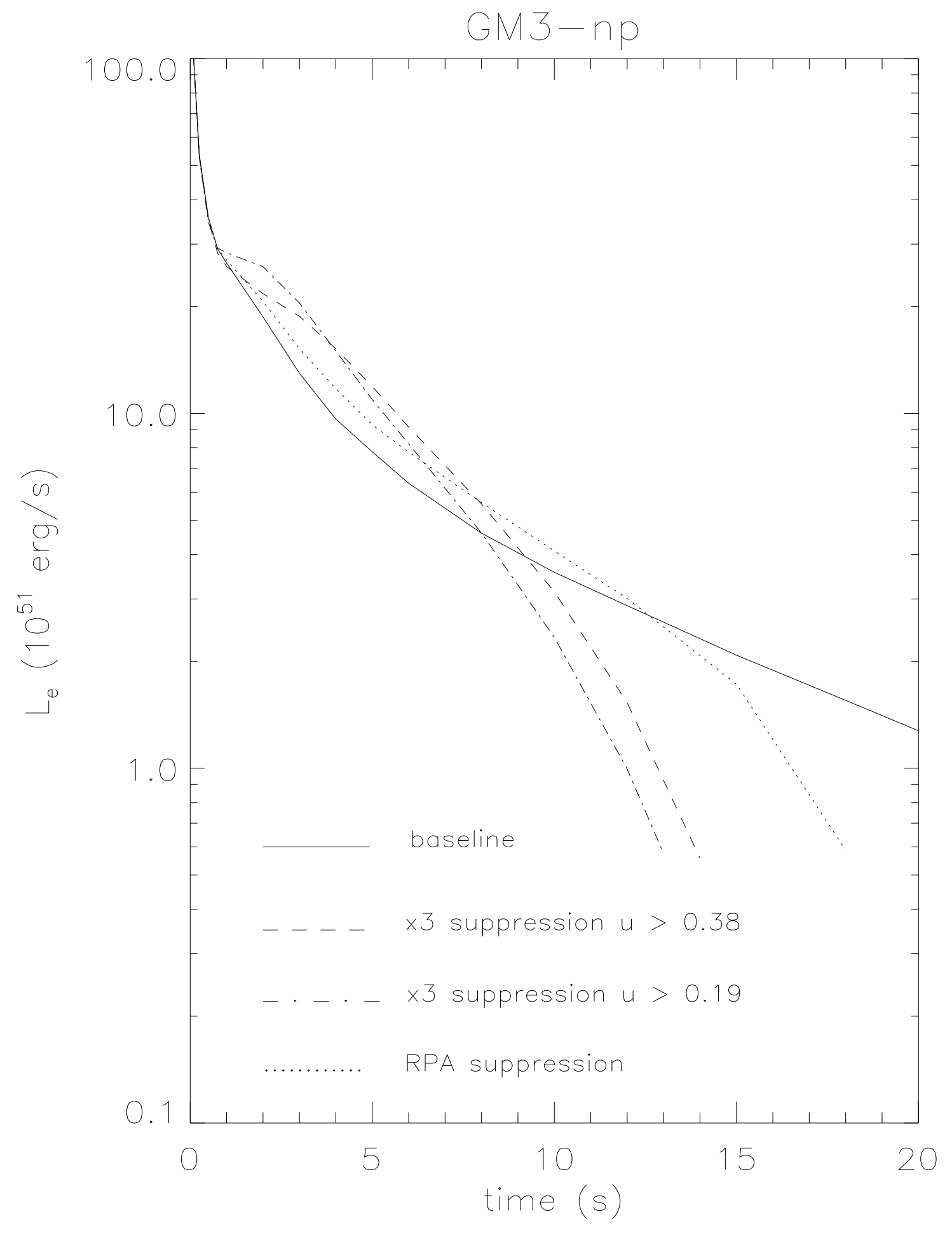

FIG. 35. 